Supporting Information for

\title{
Activation of an Open Shell, Carbyne-Bridged Diiron Complex Toward Binding of Dinitrogen
}

\author{
Charles H. Arnett and Theodor Agapie* \\ Division of Chemistry and Chemical Engineering, California Institute of Technology, Pasadena, \\ California 91125, United States
}

\section{Table of Contents}

Experimental and Synthetic Details................................................2-4

Synthetic Procedures and Characterization ......................................... 5-33

Compound 1......................................................................

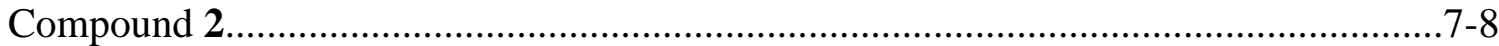

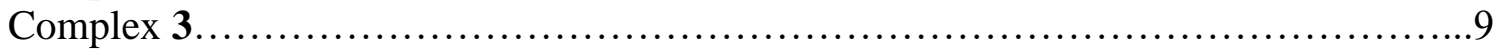

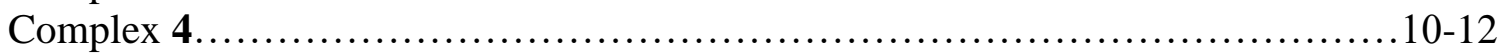

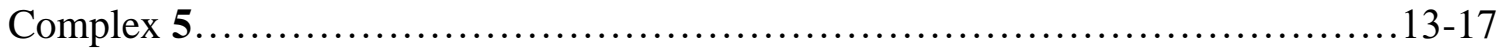

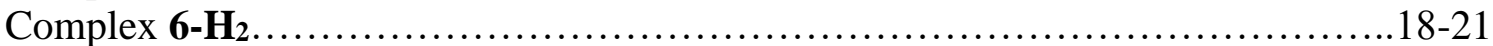

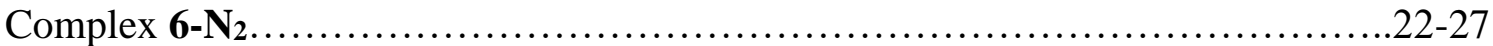

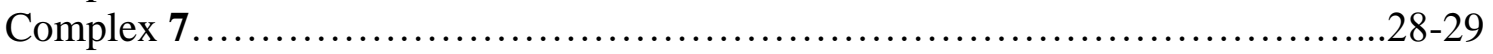

Complex 8...................................................................

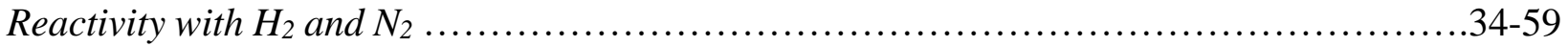

DFT Calculations............................................................ $60-72$

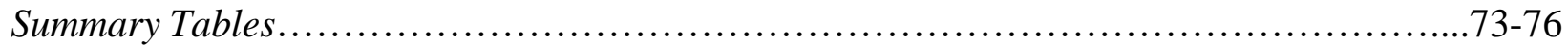

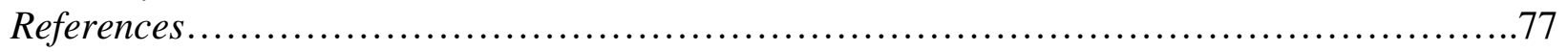




\section{Experimental and Synthetic Details}

\section{General Considerations}

All reactions were performed at room temperature in a nitrogen filled $\mathrm{M}$. Braun glovebox or using standard Schlenk techniques unless otherwise specified. Glassware was oven dried at $140^{\circ} \mathrm{C}$ for at least two hours prior to use, and allowed to cool under vacuum. $\operatorname{Bis}(o-$ diisopropylphosphinophenyl)-chlorophosphine $\left(\mathrm{P}_{2} \mathrm{P}^{\mathrm{Cl}}\right)$ was prepared as described elsewhere. ${ }^{1}$ All other reagents were obtained commercially unless otherwise noted and typically stored over activated $4 \AA$ molecular sieves. Tetrahydrofuran, toluene- $d_{8}$ and benzene- $d_{6}$ were dried using sodium/benzophenone ketyl, degassed with three freeze-pump-thaw cycles, vacuum transferred, and stored over $3 \AA$ molecular sieves prior to use. Diethyl ether, benzene, toluene, acetonitrile, hexanes, and pentane were dried by sparging with nitrogen for at least 15 minutes, then passing through a column of activated A2 alumina under positive nitrogen pressure. ${ }^{1} \mathrm{H}$ and ${ }^{31} \mathrm{P}$ NMR spectra were recorded on a Varian 300 or $400 \mathrm{MHz}$ spectrometer. All chemical shifts $(\delta)$ are reported in ppm, and coupling constants $(J)$ are in hertz. The ${ }^{1} \mathrm{H}-\mathrm{NMR}$ spectra were referenced using residual $\mathrm{H}$ impurity in the deuterated solvent. UV-Vis spectra were recorded on a Varian Cary Bio 50 spectrophotometer. Infrared (ATR-IR) spectra were recorded on a Bruker ALPHA ATR-IR spectrometer. Elemental analyses were performed at Caltech.

\section{Physical Methods}

Mössbauer Measurements. Zero field ${ }^{57} \mathrm{Fe}$ Mössbauer spectra were recorded in constant acceleration on a spectrometer from See Co (Edina, MN) equipped with an SVT-400 cryostat (Janis, Wilmington, WA). The quoted isomer shifts are relative to the centroid of the spectrum of $\alpha-\mathrm{Fe}$ foil at room temperature. Unless otherwise noted, samples were prepared by grinding polycrystalline (20-50 mg) into a fine powder and pressed into a homogenous pellet with boron nitride in a cup fitted with a screw cap. The data were fitted to Lorentzian lineshapes using the program WMOSS (www.wmoss.org).

Magnetic Measurements. Magnetic measurements for 5 were conducted with a Quantum Design MPMS3 SQUID Magnetometer at the University of California, Los Angeles. A polycrystalline sample of 5 was wrapped in plastic film and placed in a gelatin capsule. The capsule was then inserted into a plastic straw. Magnetization data at $100 \mathrm{~K}$ from 0 to $4 \mathrm{~T}$ were collected to confirm the absence of ferromagnetic impurities. Direct current variable temperature magnetic susceptibility measurements were collected between 1.8 and $300 \mathrm{~K}$ with a $0.1 \mathrm{~T}$ field. Magnetic susceptibility data was corrected for diamagnetism of the sample, estimated using Pascal's constants. Magnetic susceptibility data was simulated with PHI. ${ }^{2}$

X-ray Crystallography. For compounds 4-8, low-temperature $(100 \mathrm{~K})$ diffraction data $(\varphi$ and $\omega$-scans) were collected on a Bruker AXS D8 VENTURE KAPPA diffractometer coupled to a PHOTON $100 \mathrm{CMOS}$ detector with Mo K $\alpha$ radiation $(\lambda=0.71073 \AA)$ or with $\mathrm{Cu} \mathrm{K} \alpha(\lambda=1.54178$ $\AA$ ). All diffractometer manipulations, including data collection, integration, and scaling were carried out using the Bruker APEXII software. ${ }^{3}$ Absorption corrections were applied using SADABS. $^{4}$ Structures were solved by direct methods using SHELXS ${ }^{5}$ and refined against $F 2$ on all data by full-matrix least squares with SHELXL-2014 ${ }^{6}$ interfaced with Olex2-1.2.8 ${ }^{7}$ and using established refinement techniques. All non-hydrogen atoms were refined anisotropically, except heavily disordered solvent in some cases. Hydrogen atoms were included into the model at geometrically calculated positions and refined using a riding model, except for the hydride ligands in $\mathbf{5}, \mathbf{7}$ and $\mathbf{8}$ and the carbene $\mathrm{C}-H$ in $\mathbf{8}$. The isotropic displacement parameters of all hydrogen 
atoms were fixed to 1.2 times the $U$ value of the atoms they are linked to $(1.5$ times for methyl groups).

Special Refinement Details for $\left(\mathrm{P}_{6} \mathrm{ArCH}_{3}\right) \mathrm{Fe}_{2} \mathrm{Br}_{2}$. Compound 4 crystallizes in the monoclinic space group $C 2 / c$ with half of the molecule in the asymmetric unit. The molecule crystallizes with a two-fold rotation axis along the $\mathrm{C} 1-\mathrm{C} 2$ bond, such that the hydrogen substituents of the methyl group are disordered over a special position. These were refined with an AFIX 133 command.

Special Refinement Details for $\left(\mathrm{P}_{6} \mathrm{ArC}\right) \mathrm{Fe}_{2} \mathrm{H}$. Compound 5 crystallizes in the monoclinic space group $C 2 / c$ with half of the molecule in the asymmetric unit. The data was of sufficient quality to unambiguously locate the bridging hydride ligand in the Fourier difference map.

Special Refinement Details for $\left(\mathrm{P}_{6} \mathrm{ArCH}_{3}\right) \mathrm{Fe}_{2}\left(\mathrm{~N}_{2}\right)_{2}(\mathrm{H})_{4}$. Compound 6- $\mathbf{N}_{2}$ crystallizes in the monoclinic space group $C 2 / c$ with half of the molecule in the asymmetric unit. The molecule crystallizes with a two-fold rotation axis along the $\mathrm{C} 1-\mathrm{C} 2$ bond, such that the hydrogen substituents of the methyl group are disordered over a special position. These were refined with an AFIX 133 command. There is one isopropyl group which is also disordered over two positions. Electron density corresponding to the hydride ligands could located in the Fourier diffraction map. However, stable refinement with reasonable bond lengths required imposing a fixed $\mathrm{Fe}-\mathrm{H}$ distance of 1.54(2) $\AA$. There is residual electron density close to the iron centers, but they are too close $(\sim 0.8 \AA)$ to be another atom. The possibility of non-merohedral twinning was evaluated through cell_now, but a significant twin component could not be identified. While the residual density could be due to disorder which cannot be satisfactorily modelled, their proximity to the heavy metals suggests they may arise due to absorption problems or truncation errors instead.

Special Refinement Details for $\left[\mathrm{P}_{6} \mathrm{ArCH}_{3}\right](\mathrm{FeH})_{2}\left(\mu-\mathrm{N}_{2}\right)$. Compound 7 crystallizes in the orthorhombic space group $\mathrm{Pbca}$ with one molecule in the asymmetric unit along with one molecule of co-crystallized pentane. The co-crystallized pentane is disordered over two positions (52\% and $48 \%$ relative occupancies. Electron density corresponding to two hydride ligands could be located directly in the Fourier difference map, which were refined with the aid of a similarity restraint on their 1,2-distances to Fe1 and Fe2, respectively.

Special Refinement Details for $\left(\mathrm{P}_{6} \mathrm{ArCH}\right) \mathrm{Fe}_{2}\left(\mathrm{~N}_{2}\right)_{2}(\mathrm{H})_{2}$. Compound 8 crystallizes in the monoclinic space group $P 2_{1} / n$ with one molecule in the asymmetric unit. Electron density corresponding to two hydride ligands could be located directly in the Fourier difference map, which were refined with the aid of a similarity restraint on their 1,2-distances to Fe2. The difference map also revealed electron density corresponding to one proton on $\mathrm{C} 1$, which was allowed to freely refine.

\section{DFT Calculations}

Calculations were carried out using version 4.0.2 of the ORCA package. ${ }^{8}$ Gas phase geometry optimizations were conducted using both the BP86 and TPSS functionals in combination with the scalar relativistically recontracted versions of the def2-SVP (ZORA-def2-SVP) basis set on most $\mathrm{C}$ and $\mathrm{H}$ atoms. An enlarged basis set (ZORA-def2-TZVP) was employed for the Fe and $\mathrm{P}$ atoms, the iron-bound $\mathrm{C}$ and $\mathrm{H}$ ligands as well as any $\mathrm{C}$ atoms of the central aryl linker which undergo significant distortion in the solid state structure. For all atoms, the general-purpose segmented allelectron relativistically contracted auxiliary Coulomb-fitting basis (SARC/J) was employed. Optimizations were followed by a frequency calculation to ensure a true minimum. In general, geometries obtained using the meta-GGA functional TPSS correlated better with the solid state structure. As such, single point calculations were carried out on these optimized geometries, using either the TPSSh, PBE0 or B3LYP functionals and an enlarged basis set (ZORA-def2-TZVPP) on 
the $\mathrm{Fe}$ and $\mathrm{P}$ atoms, the iron-bound $\mathrm{C}$ and $\mathrm{H}$ ligands as well as any $\mathrm{C}$ atoms of the central aryl linker which undergo significant distortion in the solid state structure. For all functionals employed, the $S=1$ state was predicted to be lowest in energy, consistent with experiment. Orbital and spin density plots were rendered using UCSF Chimera. ${ }^{9}$

For DFT calculations of Mössbauer parameters, ${ }^{10}$ the TPSSh functional was used in combination with the def2-TZVP basis set on most $\mathrm{C}$ and $\mathrm{H}$ atoms. The CP(PPP) basis set was employed for $\mathrm{Fe}$ and the IGLO-III basis set was utilized for $\mathrm{P}$ and the iron-bound $\mathrm{C}$ and $\mathrm{H}$ ligands as well as any $\mathrm{C}$ atoms of the central aryl linker which undergo significant distortion in the solid state structure. The general purpose def $2 / \mathrm{J}$ Coulomb fitting basis was employed on atoms using the def2TZVPbasis, while the AutoAux feature of ORCA was used to generate auxiliary bases for the other atoms. All auxiliary bases were fully decontracted. To capture core polarization effects, the radial integration accuracy was increased around the $\mathrm{Fe}, \mathrm{P}$, and the iron-bound $\mathrm{C}$ and $\mathrm{H}$ ligands (IntAcc 7). A previously-reported calibration ${ }^{11}$ was used to convert the computed Fe core electron density to the isomer shift $(\delta)$ in units of $\mathrm{mm} \mathrm{s}^{-1}$; estimates of the uncertainty in the computed values of $\delta$ and $\Delta E_{\mathrm{Q}}$ were obtained from this calibration. The Mössbauer parameters calculated from the $S=$ 1 geometry correlated best with the experimental data. 


\section{Synthetic Procedures and Characterization}

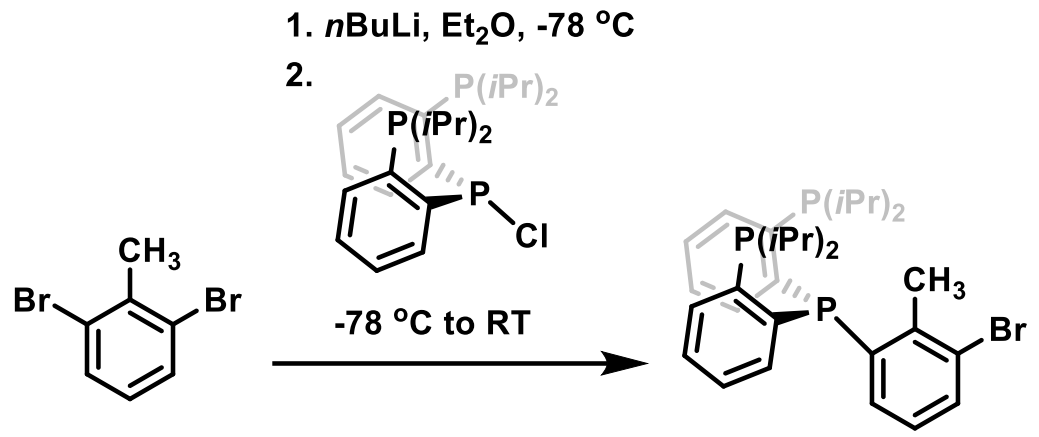

(3-bromo-2-methylphenyl)(bis(2-diisopropylphenylphosphino)phosphine, (1). A solution of $n \mathrm{BuLi}$ (2.4 mL, $3.87 \mathrm{mmol}, 1.05$ equiv.) was added dropwise to stirring solution of 2,6dibromotoluene $\left(919 \mathrm{mg}, 3.68 \mathrm{mmol}, 1\right.$ equiv.) in $35 \mathrm{~mL}$ of diethyl ether at $-78^{\circ} \mathrm{C}$. After stirring for 105 minutes at $-78{ }^{\circ} \mathrm{C}$, the resulting colorless suspension was removed from the cold bath and stirred for an additional 7 minutes. The resulting colorless solution was then cooled back to -78 ${ }^{\circ} \mathrm{C}$. CAUTION: DO NOT CONCENTRATE TO DRYNESS! The monolithiated species reacts exothermically if concentrated to dryness, causing glass failure and implosion of the reaction flask. A suspension of bis(o-diisopropylphosphinophenyl)-chlorophosphine $(1.76 \mathrm{~g}, 3.87 \mathrm{mmol}, 1.05$ equiv.) in $12 \mathrm{~mL}$ of toluene was then added dropwise. The cold bath was removed and the orange suspension was allowed to warm to room temperature. After stirring for three hours, the resulting pale yellow suspension was filtered over Celite and concentrated in vacuo. The residue was washed with copious amounts of acetonitrile followed by pentane $(2 \times 5 \mathrm{~mL})$ to afford $\mathbf{1}$ as an off-white powder $\left(1.70 \mathrm{~g}, 78 \%\right.$ yield). ${ }^{1} \mathrm{H}$ NMR $\left(400 \mathrm{MHz}, \mathrm{C}_{6} \mathrm{D}_{6}\right) \delta=7.41(\mathrm{~d}, J=8.1 \mathrm{~Hz}, 1 \mathrm{H}), 7.31(\mathrm{~m}, 2 \mathrm{H})$, $7.06(\mathrm{t}, J=7.0 \mathrm{~Hz}, 2 \mathrm{H}), 6.97(\mathrm{~m}, 2 \mathrm{H}), 6.91(\mathrm{t}, J=7.6 \mathrm{~Hz}, 3 \mathrm{H}), 6.59(\mathrm{t}, J=7.7 \mathrm{~Hz}, 1 \mathrm{H}), 2.72(\mathrm{~s}$, $3 \mathrm{H}), 2.11(\mathrm{~m}, 2 \mathrm{H}), 1.95(\mathrm{~m}, 2 \mathrm{H}), 1.12(\mathrm{~m}, 12 \mathrm{H}), 0.87(\mathrm{~m}, 12 \mathrm{H}) .{ }^{31} \mathrm{P} \mathrm{NMR}\left(162 \mathrm{MHz}, \mathrm{C}_{6} \mathrm{D}_{6}\right) \delta=-$ $0.7(\mathrm{~d}, J=156 \mathrm{~Hz}, 2 \mathrm{P}),-17.6(\mathrm{t}, J=156 \mathrm{~Hz}, 1 \mathrm{P}) .{ }^{13} \mathrm{C} \mathrm{NMR}\left(101 \mathrm{MHz}, \mathrm{C}_{6} \mathrm{D}_{6}\right) \delta=147.32(\mathrm{~m})$, $147.06(\mathrm{~m}), 146.98(\mathrm{~m}), 142.74$ (dd, $J=76 \mathrm{~Hz}, 124 \mathrm{~Hz}), 142.28$ (t, $J=32 \mathrm{~Hz}), 142.07$ (t, $J=28$ Hz), 141.76 (s), 141.49 (s), 134.91 (s), 132.65 (s), 129.07 (b), 127.08 (b), 126.67 (d, $J=16 \mathrm{~Hz}$ ), 25.90 (b), 24.31 (b), 21.96 (s), 21.71 (s), 20.45 (m), 19.73 (b). 


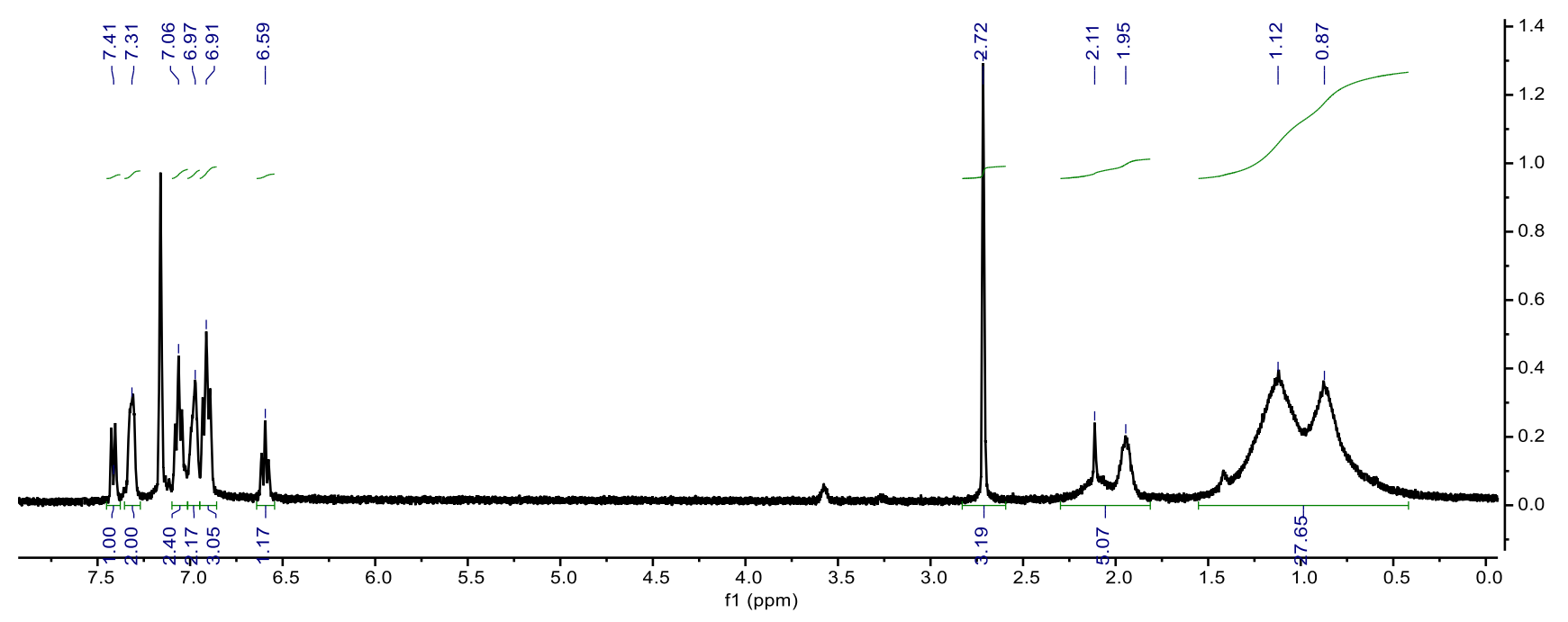

Figure S1. ${ }^{1} \mathrm{H}$ NMR (400 MHz) of 1 in $\mathrm{C}_{6} \mathrm{D}_{6}$

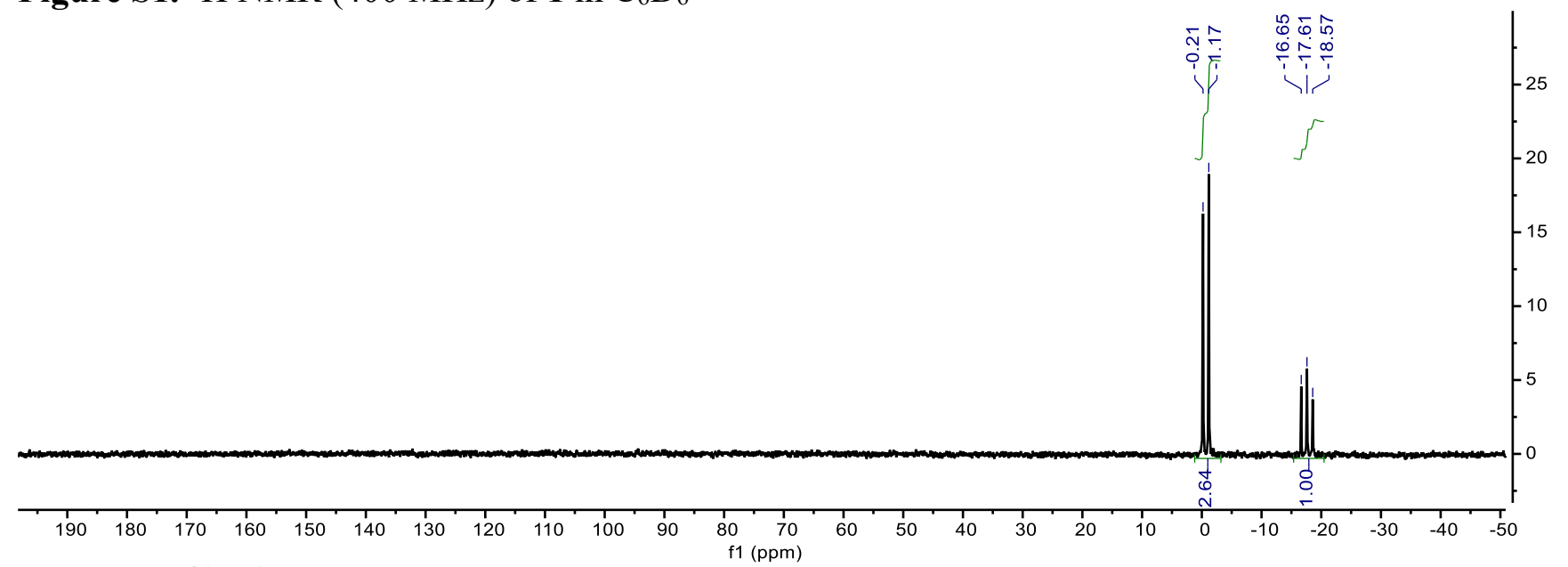

Figure S2. ${ }^{31} \mathrm{P}\left\{{ }^{1} \mathrm{H}\right\} \mathrm{NMR}(162 \mathrm{MHz})$ of 1 in $\mathrm{C}_{6} \mathrm{D}_{6}$

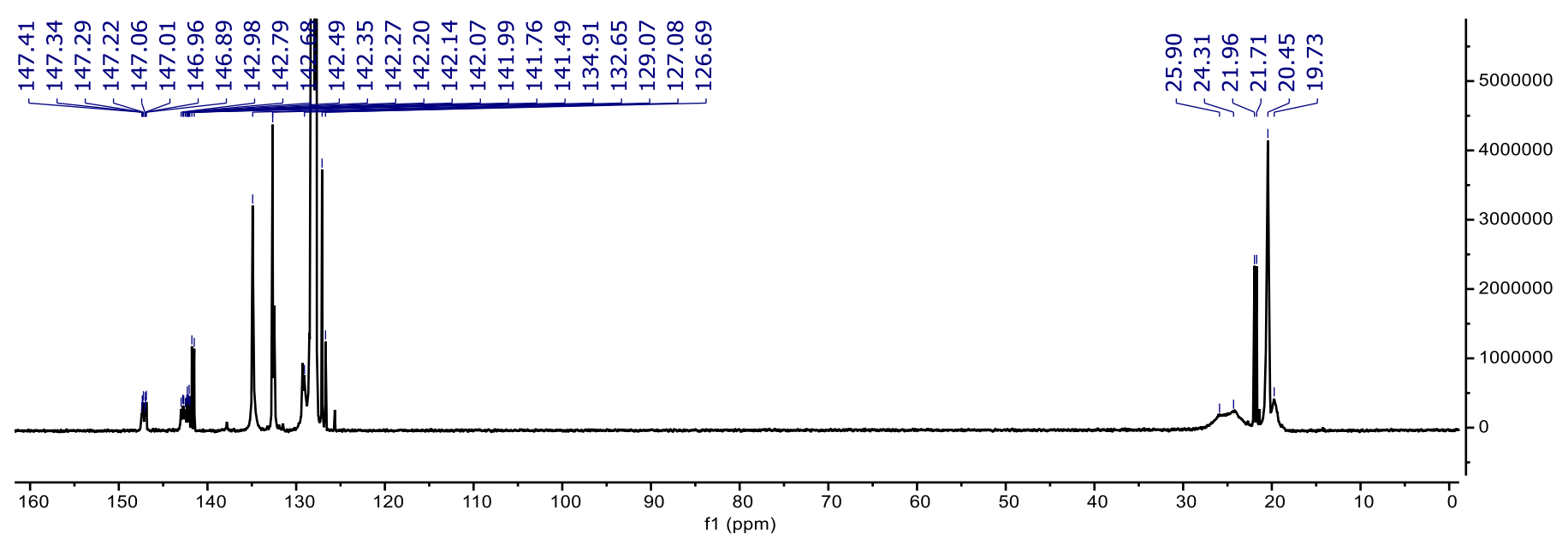

Figure S3. ${ }^{13} \mathrm{C}\left\{{ }^{1} \mathrm{H}\right\}$ NMR $(101 \mathrm{MHz})$ of $\mathbf{1}$ in $\mathrm{C}_{6} \mathrm{D}_{6}$ 


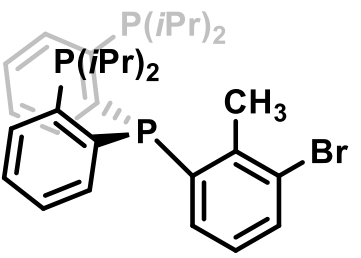

1. Mg (10 equiv.), THF

2.

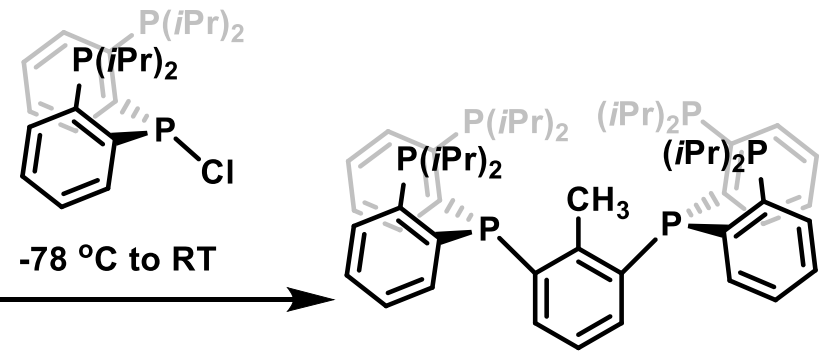

2,6-bis[bis(2-diisopropylphenylphosphino)phosphino)]toluene, (2). Excess magnesium turnings (1.94, 79.6 mmol, 10 equiv.) were added to a solution of $\mathbf{1}$ (4.67 g, $7.96 \mathrm{mmol}, 1$ equiv.) in $35 \mathrm{~mL}$ of tetrahydrofuran. After stirring for 8 hours, the solution was filtered and cooled to -78 ${ }^{\circ} \mathrm{C}$. A solution of bis(o-diisopropylphosphinophenyl)-chlorophosphine (3.78 g, $8.36 \mathrm{mmol}, 1.05$ equiv.) in $5 \mathrm{~mL}$ of tetrahydrofuran was then added dropwise. The cold bath was removed and the yellow solution was allowed to warm to room temperature. After stirring overnight, excess 1,4dioxane (20 equiv.) was added, resulting in precipitation of a colorless solid. After stirring for 30 minutes, the mixture was concentrated to dryness. The oily residue was triturated once with hexanes and reconcentrated dryness. The resulting yellow solid was extracted with toluene, affording a yellow suspension which was filtered over Celite. After concentrating en vacuo, the residue was washed with acetonitrile $(3 \times 20 \mathrm{~mL})$ and pentane $(3 \times 6 \mathrm{~mL})$, affording 2 as a white powder $\left(5.97 \mathrm{~g}, 81 \%\right.$ yield). ${ }^{1} \mathrm{H}$ NMR $\left(400 \mathrm{MHz}, \mathrm{C}_{6} \mathrm{D}_{6}\right) \delta=7.35(\mathrm{~b}, 4 \mathrm{H}), 7.20-7.00(\mathrm{~b}, 12 \mathrm{H}), 6.98$ $(\mathrm{d}, J=8 \mathrm{~Hz}, 2 \mathrm{H}), 6.79$ (t, $J=8 \mathrm{~Hz}, 1 \mathrm{H}), 3.02$ (s, 3H), $2.11(\mathrm{~b}, 4 \mathrm{H}), 1.95$ (b, 4H), 1.15 (b, 24H), $0.88(\mathrm{~b}, 24 \mathrm{H}) .{ }^{31} \mathrm{P}$ NMR $\left(162 \mathrm{MHz}, \mathrm{C}_{6} \mathrm{D}_{6}\right) \delta=-1.48(\mathrm{~d}, J=159 \mathrm{~Hz}, 4 \mathrm{P}),-20.32$ (t, $J=152 \mathrm{~Hz}$, 2P). ${ }^{13} \mathrm{C}$ NMR (101 MHz, $\left.\mathrm{C}_{6} \mathrm{D}_{6}\right) \delta=148.33(\mathrm{~m}), 147.03(\mathrm{~b}), 142.48(\mathrm{~m}), 142.53(\mathrm{~m}), 139.21(\mathrm{~m})$, 137.82 (s), 136.26 (broad s), 134.90 (broad s), 132.44 (broad s), 129.27 (s), 128.80 (broad m), 128.51 (s), 125.87 (s), 125.64 (s), 25.78 (broad m), 23.78 (broad m), 21.41 (s), 20.77 (s), 20.57 (s), $20.33(\mathrm{~m}), 19.18$ (broad m). 


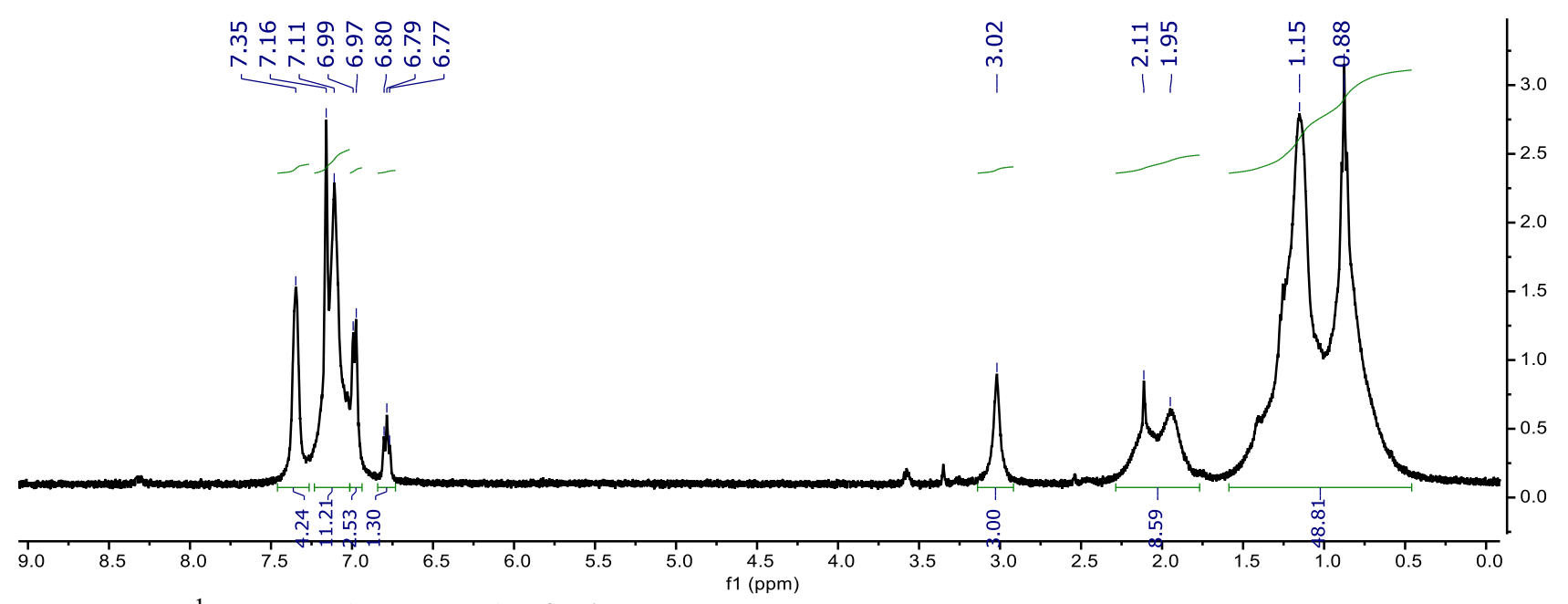

Figure S4. ${ }^{1} \mathrm{H}$ NMR (400 MHz) of 2 in $\mathrm{C}_{6} \mathrm{D}_{6}$

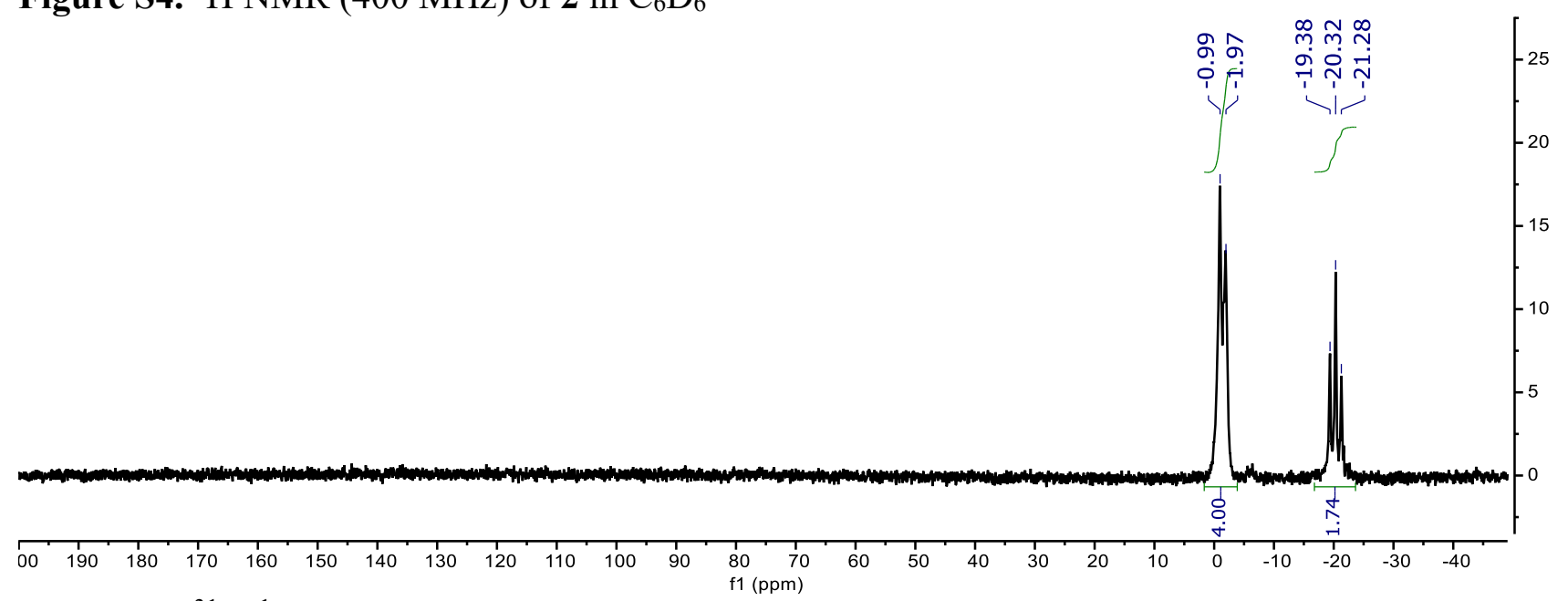

Figure S5. ${ }^{31} \mathrm{P}\left\{{ }^{1} \mathrm{H}\right\}$ NMR $(162 \mathrm{MHz})$ of 2 in $\mathrm{C}_{6} \mathrm{D}_{6}$

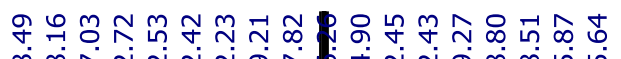

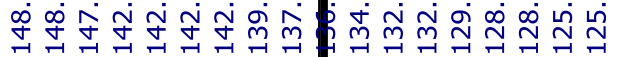

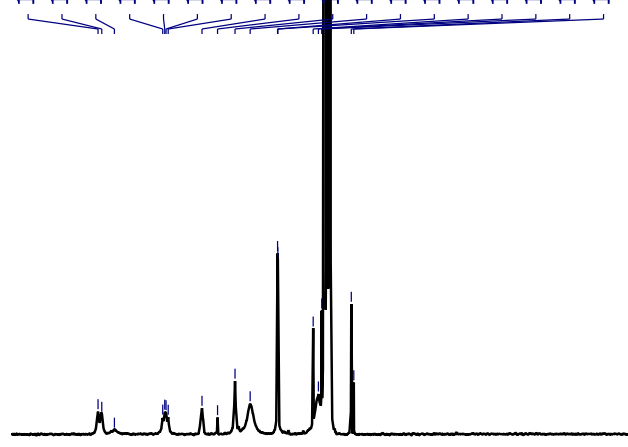

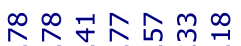

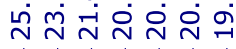

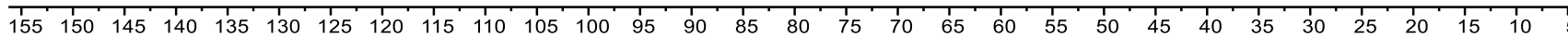

Figure S6. ${ }^{13} \mathrm{C}\left\{{ }^{1} \mathrm{H}\right\}$ NMR $(101 \mathrm{MHz})$ of 2 in $\mathrm{C}_{6} \mathrm{D}_{6}$ 


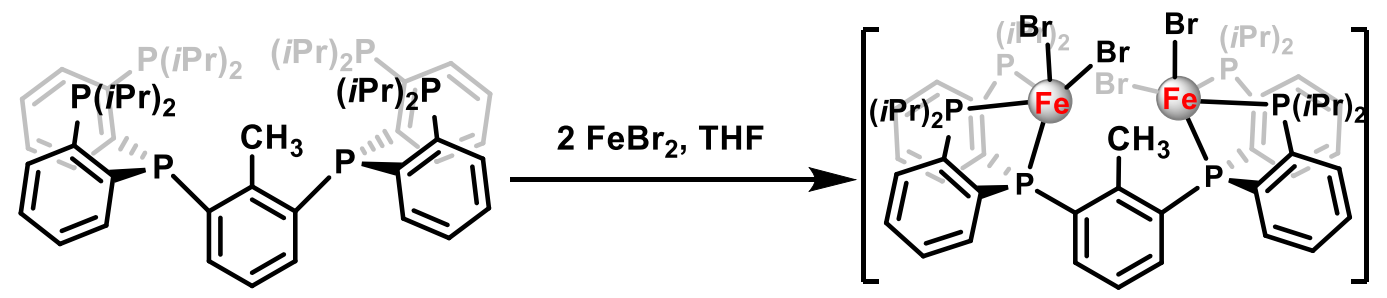

( $\left.\mathbf{P}_{6} \mathrm{ArCH}_{3}\right) \mathrm{Fe}_{2} \mathbf{B r}_{4}$, (3). A solution of $\mathbf{2}$ (5.97 g, $6.46 \mathrm{mmol}, 1.0$ equiv.) in tetrahydrofuran (10 mL) was added to a suspension of $\mathrm{FeBr}_{2}(2.78 \mathrm{~g}, 12.9 \mathrm{mmol}, 2.0$ equiv.) in tetrahydrofuran $(40 \mathrm{~mL})$. After stirring for overnight, the resulting precipitate was collected on a fine frit and washed with additional tetrahydrofuran, affording a species tentatively assigned as $\mathbf{3}$ as a yellow-green powder ( $8.23 \mathrm{~g}, 94 \%$ yield) which was used in subsequent reactions without further purification. ${ }^{1} \mathrm{H}$ NMR (400 MHz, $\mathrm{CD}_{2} \mathrm{Cl}_{2}$ ) $\delta=166.66$ (b), 161.79 (b), 71.19 (b), 44.96 (s), 38.34 (b), 28.86 (b), 16.25 (b), 14.59 (b), 14.08 (b), 12.44 (b), 11.98 (b), 10.76 (b), 8.17 (b), 6.94 (b), 2.85 (b), 0.55 (b), -8.56 (s).

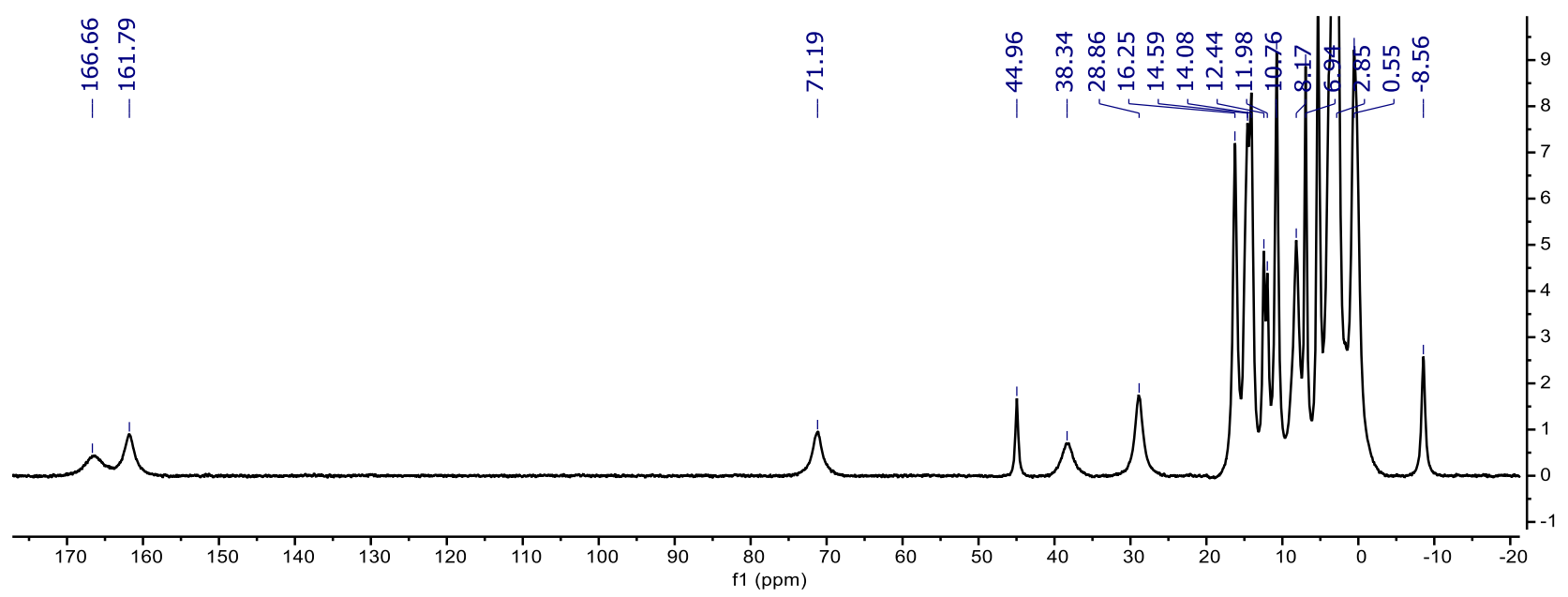

Figure S7. ${ }^{1} \mathrm{H}$ NMR (400 MHz) of $\left(\mathrm{P}_{6} \mathrm{ArCH}_{3}\right) \mathrm{Fe}_{2} \mathrm{Br}_{4}(\mathbf{3})$ in $\mathrm{CD}_{2} \mathrm{Cl}_{2}$ 


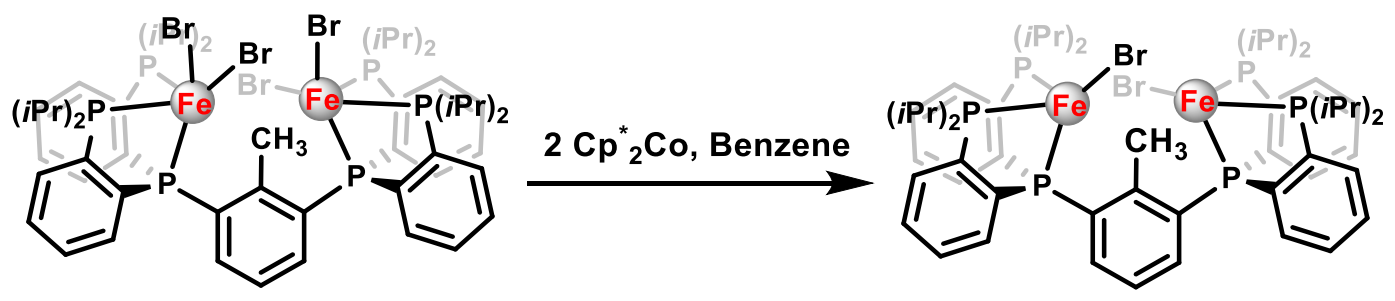

( $\left.\mathrm{P}_{6} \mathrm{ArCH}_{3}\right) \mathrm{Fe}_{2} \mathrm{Br}_{2}$ (4). A suspension of $\mathrm{Cp}_{2}{ }_{2} \mathrm{Co}$ (3.88 g, $11.8 \mathrm{mmol}, 2.0$ equiv.) in minimal tetrahydrofuran $(\sim 10 \mathrm{~mL})$ was added to a suspension of $3(7.99 \mathrm{~g}, 5.9 \mathrm{mmol}, 1.0$ equiv.) in benzene/tetrahydrofuran $(3: 1,130 \mathrm{~mL})$. After stirring for three hours, the resulting brick red suspension was filtered over Celite and concentrated in vacuo. The residue was resuspended in 60 $\mathrm{mL}$ of benzene and stirred vigorously. Pentane $(\sim 100 \mathrm{~mL})$ was added slowly to precipitate a red solid. The precipitate was collected on a fine frit and washed with additional pentane, affording 4 as a brick red powder ( $3.4 \mathrm{~g}, 48 \%$ yield). Crystals suitable for X-ray diffraction were obtained by diffusion of pentane into a concentrated benzene solution of $4 .{ }^{1} \mathrm{H}$ NMR $\left(400 \mathrm{MHz}, \mathrm{C}_{6} \mathrm{D}_{6}\right) \delta=$ 185.84 (b), 111.73 (b), 71.82 (b), 48.77 (s), 24.69 (b), 9.84 (b), 8.40 (b), 5.48 (b), -5.02 (b), -14.10 (b), -32.32 (b). UV-Vis (THF) [ $\left.\varepsilon\left(\mathrm{M}^{-1} \mathrm{~cm}^{-1}\right)\right]: 345 \mathrm{~nm}\left(5.3 \times 10^{3}\right), 404 \mathrm{~nm}\left(5.6 \times 10^{3}\right), 845 \mathrm{~nm}(4.0$ x $10^{2}$ ). Anal. Calcd (\%) for $\mathrm{C}_{55} \mathrm{H}_{78} \mathrm{Br}_{2} \mathrm{Fe}_{2} \mathrm{P}_{6}$ : C, 55.21; H, 6.57; N, 0.00. Found: C, 55.22; H, 5.92; $\mathrm{N},-0.03$.

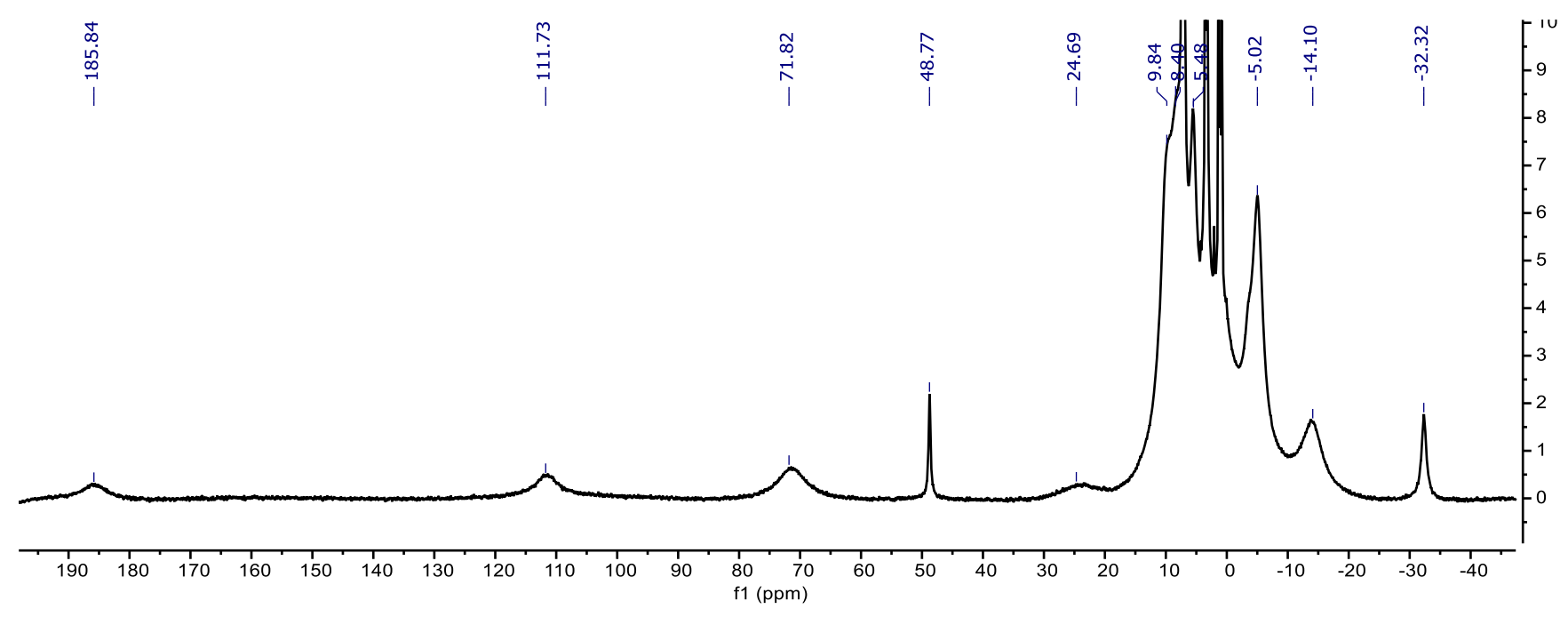

Figure S8. ${ }^{1} \mathrm{H}$ NMR (400 MHz) of $\left(\mathrm{P}_{6} \mathrm{ArCH}_{3}\right) \mathrm{Fe}_{2} \mathrm{Br}_{2}$ (4) in $\mathrm{C}_{6} \mathrm{D}_{6}$ 


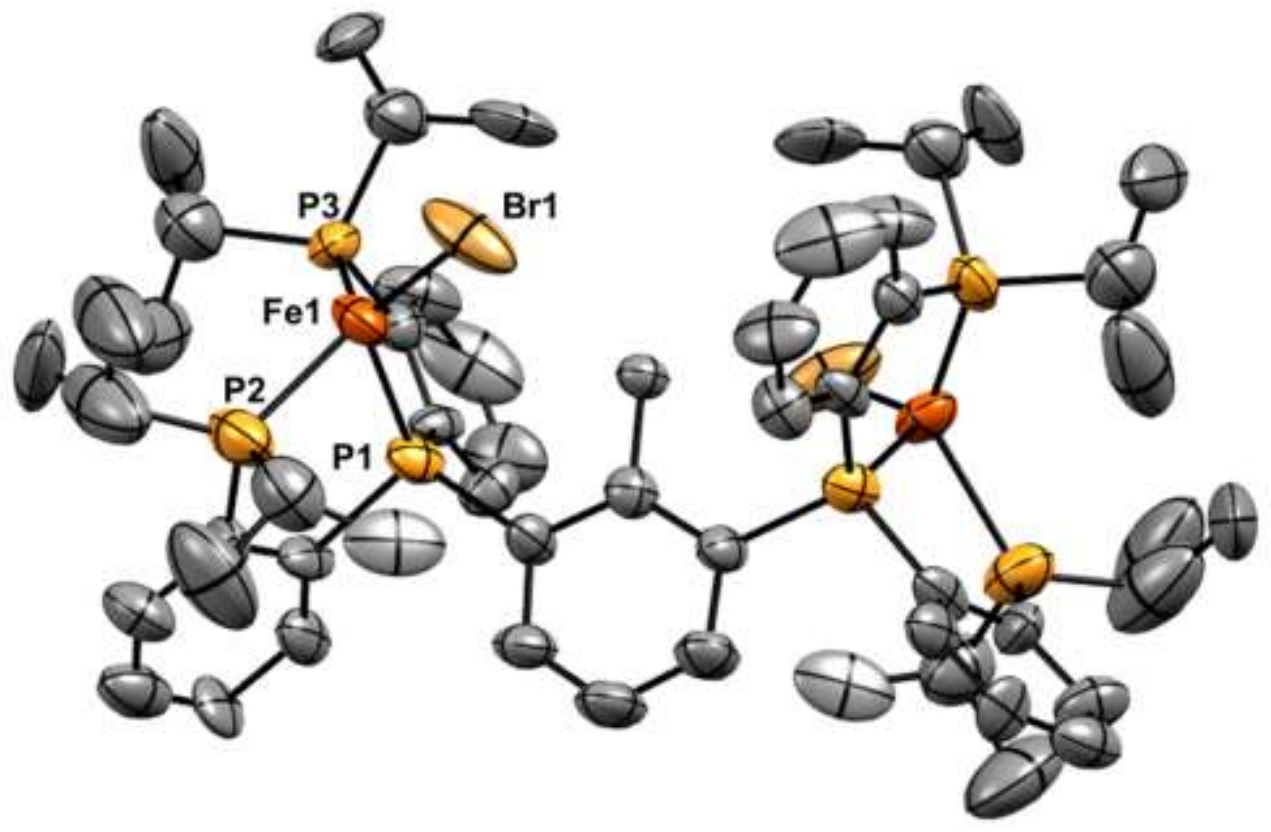

Figure S9. Crystal structure of $\left(\mathrm{P}_{6} \mathrm{ArCH}_{3}\right) \mathrm{Fe}_{2} \mathrm{Br}_{2}$ (4). Ellipsoids are shown at the $50 \%$ probability level. Hydrogen atoms and co-crystallized solvent molecules are not shown for clarity.

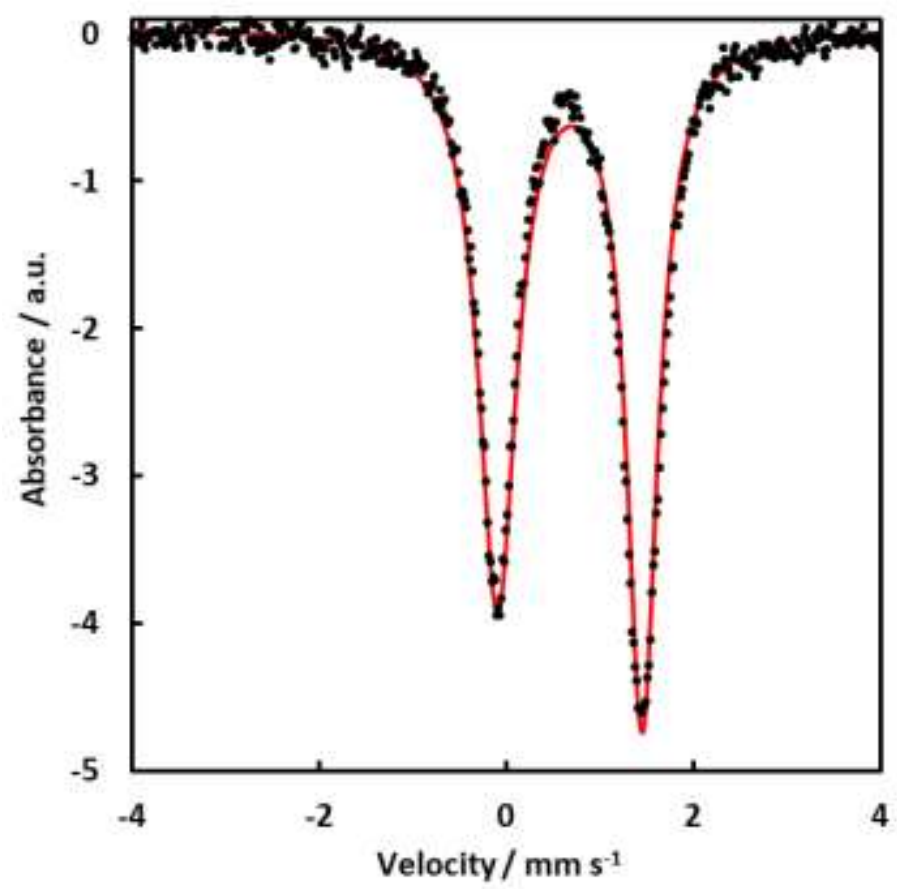

Figure S10. Solid-state ${ }^{57} \mathrm{Fe}$ Mössbauer spectrum of $\left(\mathrm{P}_{6} \mathrm{ArCH}_{3}\right) \mathrm{Fe}_{2} \mathrm{Br}_{2}$ (4) collected at $80 \mathrm{~K}$ with a $50 \mathrm{mT}$ field applied parallel to the $\gamma$ rays. Data presented in black points, simulation represented by solid red line. Fit with $\delta=0.68 \mathrm{~mm} \mathrm{~s}^{-1},\left|\Delta E_{\mathrm{Q}}\right|=1.55 \mathrm{~mm} \mathrm{~s}^{-1}, \Gamma_{\mathrm{L}}=0.50 \mathrm{~mm} \mathrm{~s}^{-1}, \Gamma_{\mathrm{R}}=0.41 \mathrm{~mm}$ $\mathrm{s}^{-1}$. 


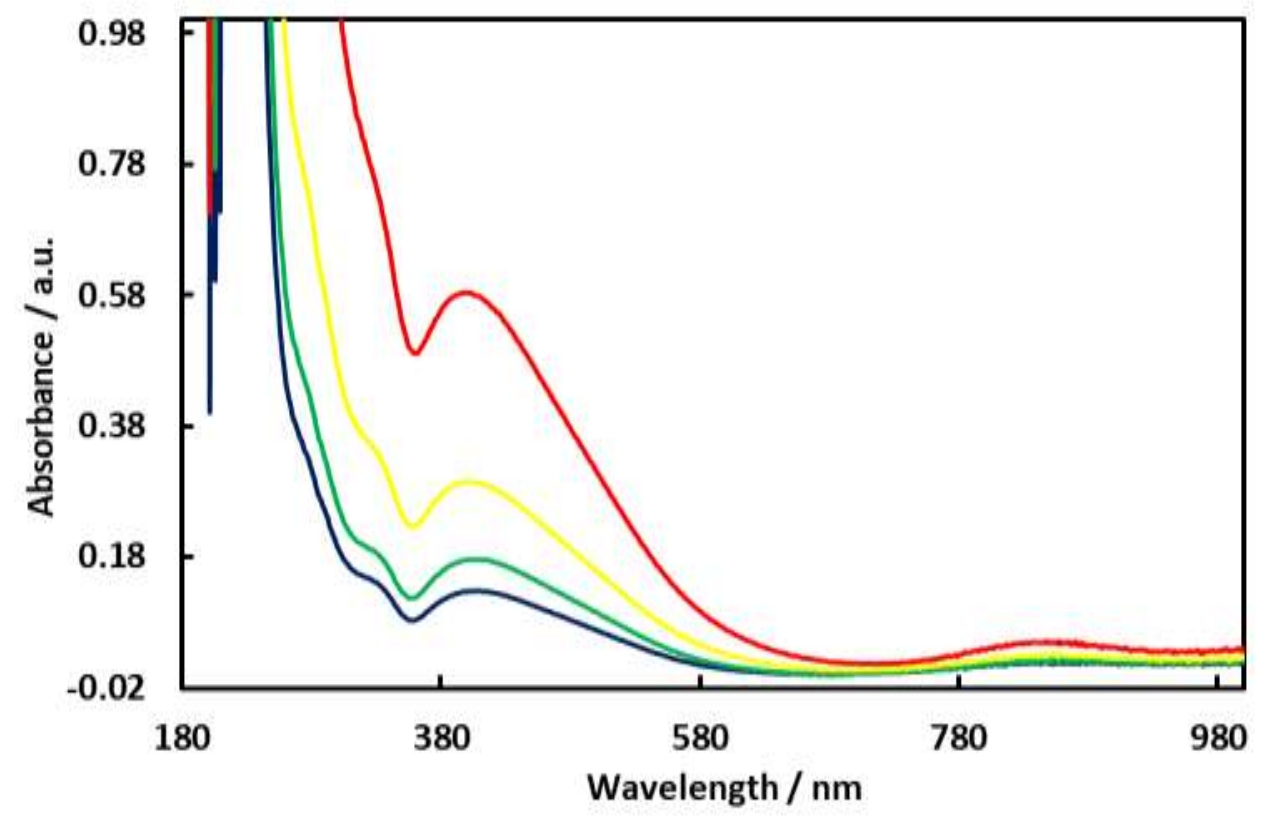

Figure S11. UV-Vis spectra of $\left(\mathrm{P}_{6} \mathrm{ArCH}_{3}\right) \mathrm{Fe}_{2} \mathrm{Br}_{2}(4)$ collected in THF at concentrations of $21 \mu \mathrm{M}$ (blue), $33 \mu \mathrm{M}$ (green), $54 \mu \mathrm{M}$ (yellow) and $109 \mu \mathrm{M}$ (red).

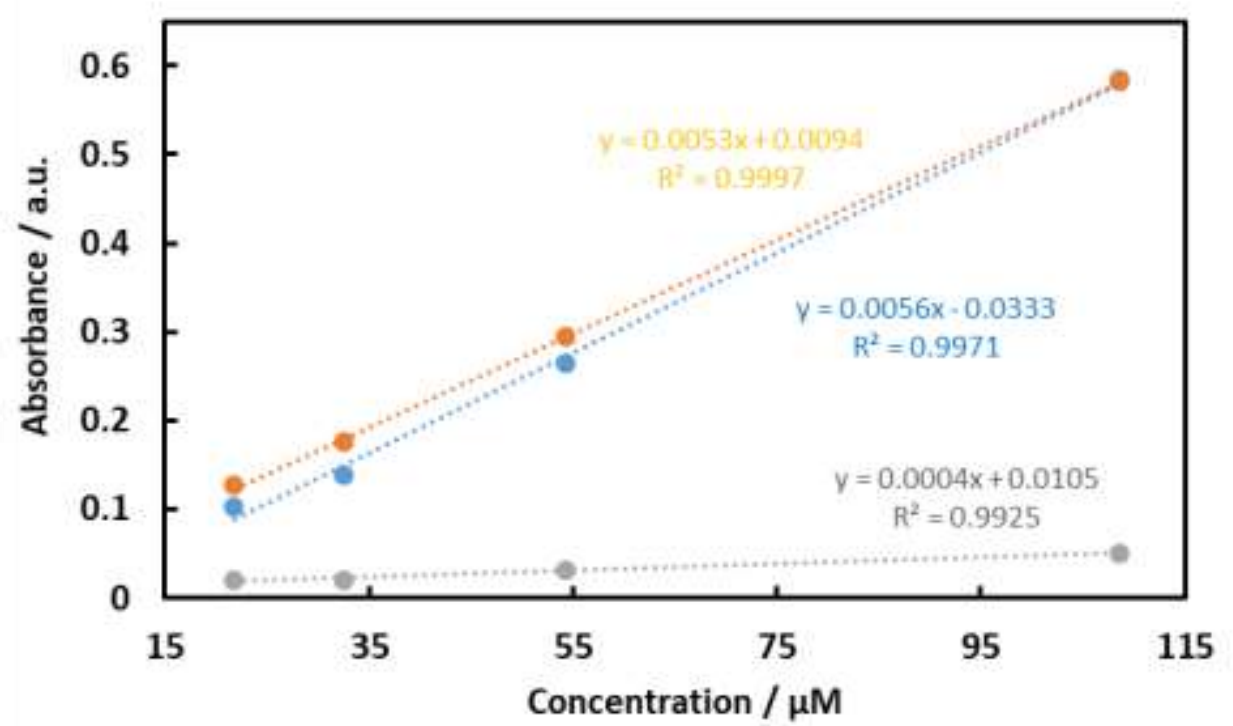

Figure S12. Beer's Law Plot for $\left(\mathrm{P}_{6} \mathrm{ArCH}_{3}\right) \mathrm{Fe}_{2} \mathrm{Br}_{2}$ (4) at $345 \mathrm{~nm}$ (orange), $404 \mathrm{~nm}$ (blue), and 845 nm (grey). 

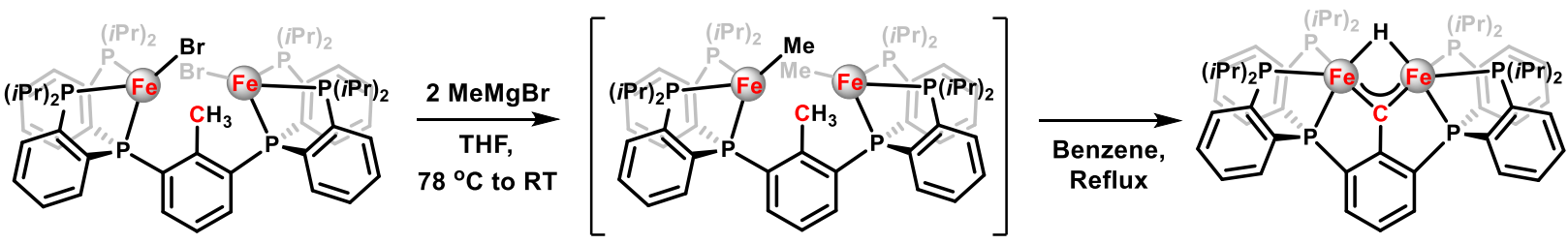

(P6ArC)Fe 2 (H) (5). A solution of 4 (443.3 $\mathrm{mg}, 0.37 \mathrm{mmol}, 1.0$ equiv.) in $12 \mathrm{~mL}$ of tetrahydrofuran was chilled to $-78^{\circ} \mathrm{C}$ in a dry ice/acetone bath. A diluted solution of $\operatorname{MeMgBr}(0.3 \mathrm{M}, 1.9 \mathrm{~mL}, 0.74$ mmol, 2.0 equiv.) was added dropwise and the mixture was stirred for 4 hours at $-78^{\circ} \mathrm{C}$ before the cold bath was removed. After stirring 20 hours at room temperature, the resulting brown solution was concentrated en vacuo. The residue was extracted with benzene and filtered over Celite. The filtrate was then charged into a Schlenk tube and heated at reflux for 24 hours. Excess 1,4-dioxane $(1 \mathrm{~mL})$ was then added. After stirring for 30 minutes, the solution was filtered over Celite and then concentrated to dryness. The residue was extracted with toluene, filtered over Celite and then concentrated under vacuum. The residue was then washed with pentane $(4 \times 8 \mathrm{~mL})$ and diethyl ether $(2 \times 8 \mathrm{~mL})$ and then eluted with copious amounts of benzene/pentane $(1 / 1)$. The filtrate was concentrated en vacuo, affording 5 as a black-brown powder $(220 \mathrm{mg}, 56 \%)$. X-ray quality crystals can be obtained by diffusion of pentane vapors into a concentrated solution of $\mathbf{5}$ in benzene. We note that on occasion $\mathbf{4}$ is not completely consumed in the reaction. In that case, the crude mixture obtained after thermolysis was stirred for 4 hours over excess $\mathrm{Na} / \mathrm{Hg}(2 \%)$ and then worked up as described. ${ }^{1} \mathrm{H}$ NMR (400 MHz, $\mathrm{C}_{6} \mathrm{D}_{6}$ ) $\delta=42.18$ (b), 35.47 (b), 28.38 (b), 12.05 (b), 10.39 (b), 9.20 (b), 5.45 (b), 4.66 (b), 4.25 (b), 0.09 (b), -2.28 (b), -15.28 (b). UV-Vis (THF) [ $\left.\varepsilon\left(\mathrm{M}^{-1} \mathrm{~cm}^{-1}\right)\right]: 313$ $\mathrm{nm}\left(1.51 \times 10^{4}\right), 366 \mathrm{~nm}\left(1.46 \times 10^{4}\right), 479 \mathrm{~nm}\left(8.7 \times 10^{3}\right), 788 \mathrm{~nm}\left(3.9 \times 10^{3}\right)$. Anal. Calcd $(\%)$ for $\mathrm{C}_{55} \mathrm{H}_{76} \mathrm{Fe}_{2} \mathrm{P}_{6}: \mathrm{C}, 63.84 ; \mathrm{H}, 7.40 ; \mathrm{N}, 0.00$. Found: C, 63.20; H, 6.99; N, 0.02 .

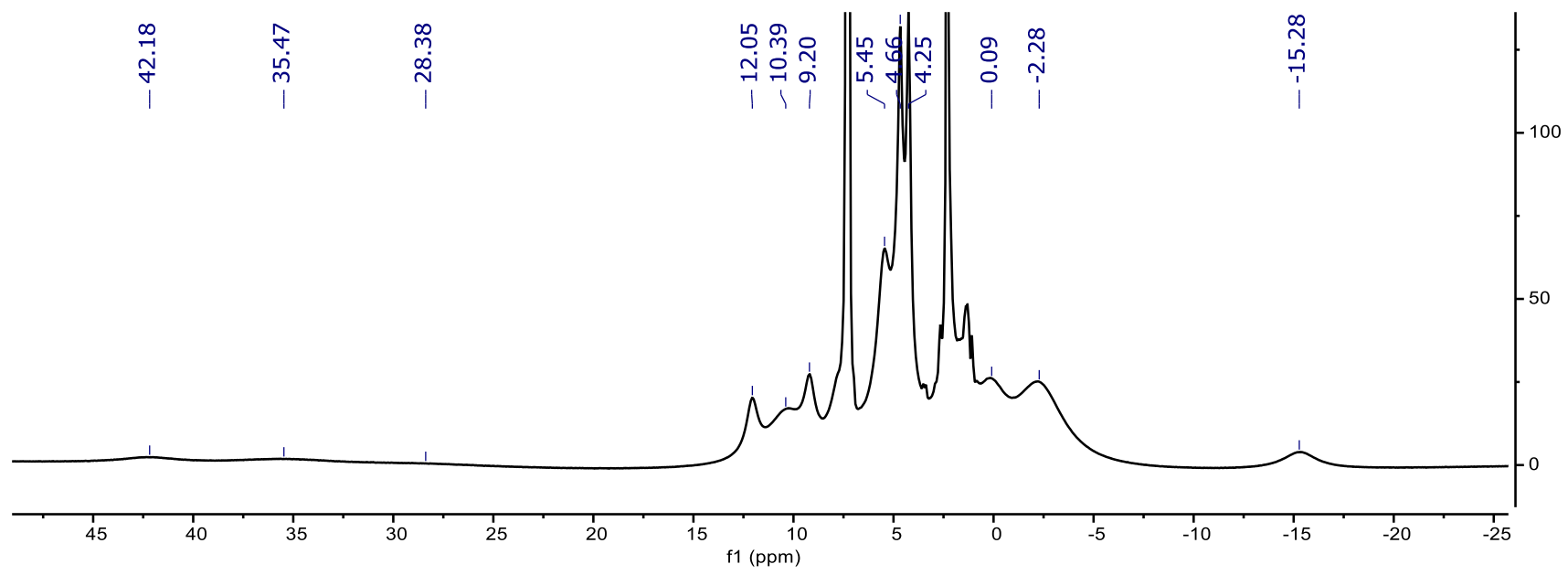

Figure S13. ${ }^{1} \mathrm{H}$ NMR (400 MHz) of $\left(\mathrm{P}_{6} \mathrm{ArC}\right) \mathrm{Fe}_{2} \mathrm{H}(\mathbf{5})$ in $\mathrm{C}_{6} \mathrm{D}_{6}$. 


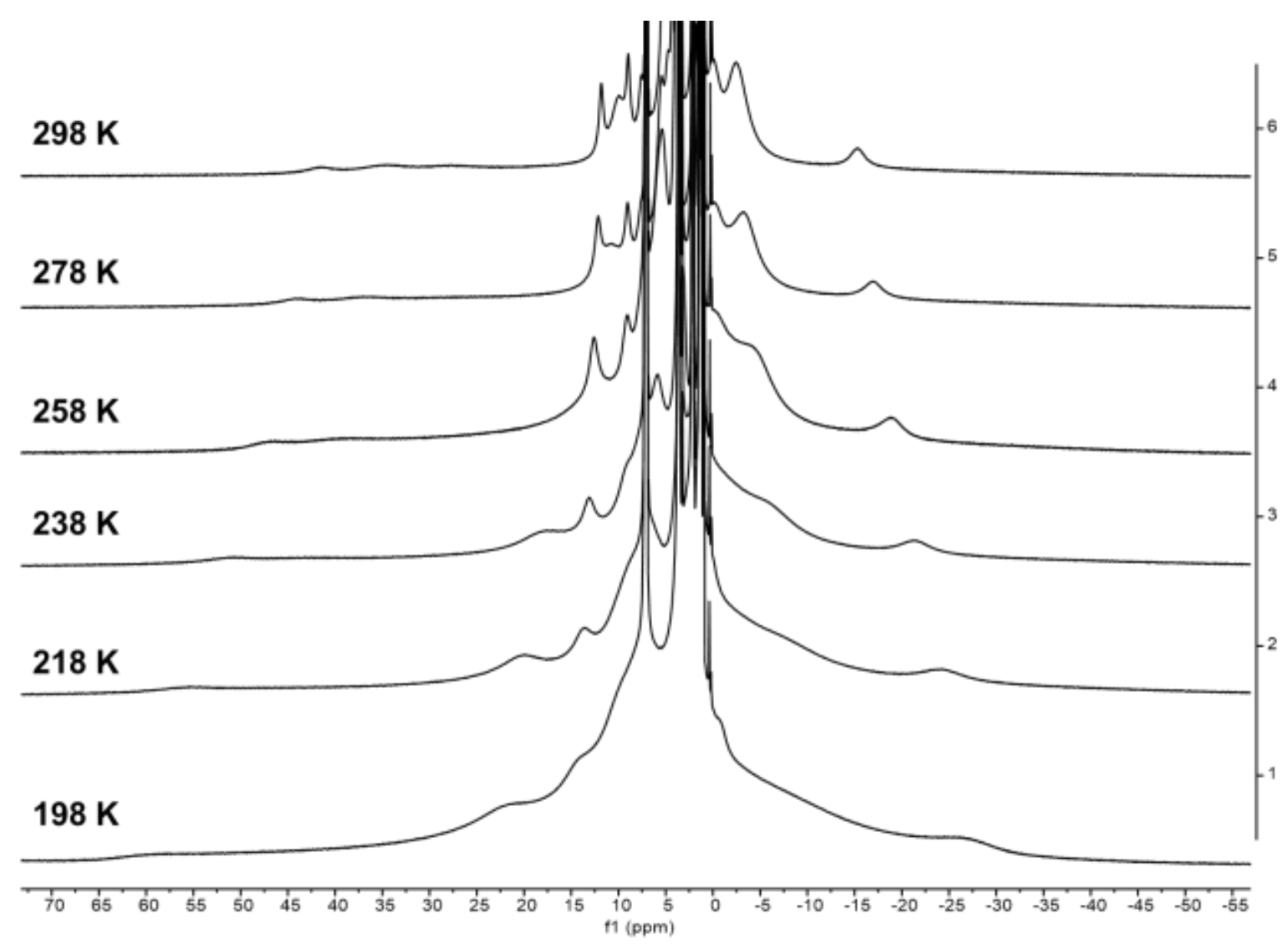

Figure S14. Variable Temperature ${ }^{1} \mathrm{H}$ NMR $(400 \mathrm{MHz})$ of $\left(\mathrm{P}_{6} \mathrm{ArC}\right) \mathrm{Fe}_{2} \mathrm{H}(\mathbf{5})$ in $\mathrm{C}_{7} \mathrm{D}_{8}$.

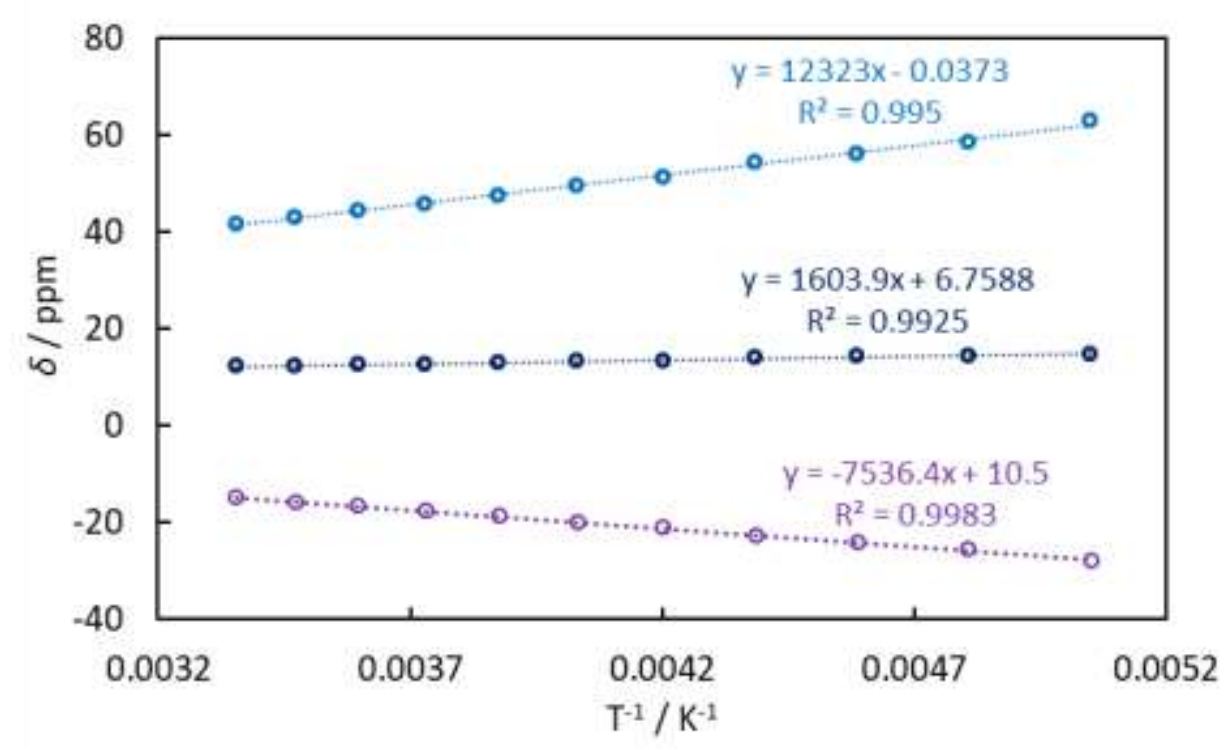

Figure S15. ${ }^{1} \mathrm{H}$ NMR chemical shifts of $\left(\mathrm{P}_{6} \mathrm{ArC}\right) \mathrm{Fe}_{2} \mathrm{H}(5)$ in $\mathrm{C}_{7} \mathrm{D}_{8}$ plotted as a function of $1 / \mathrm{T}$ display ideal Curie-behavior. 


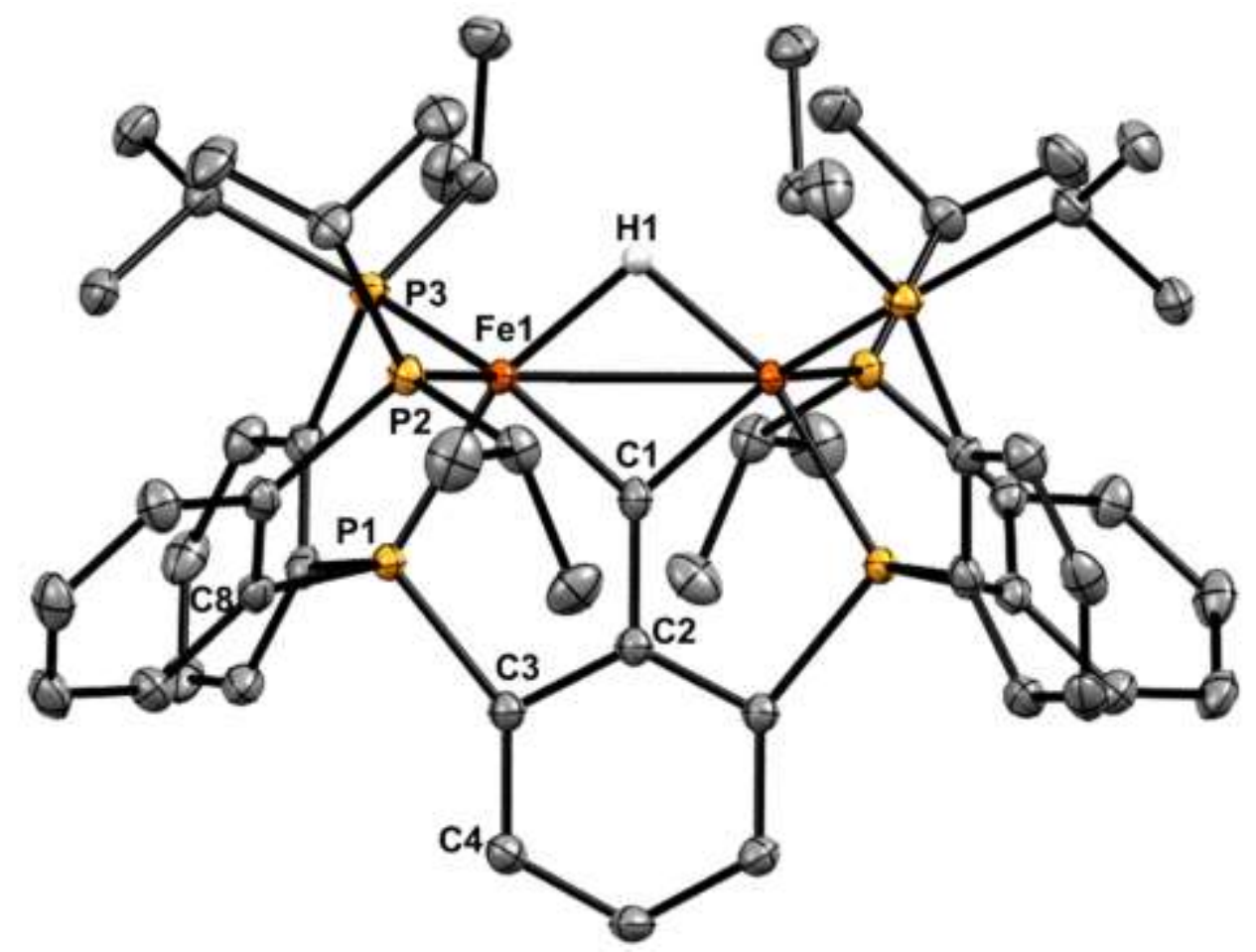

Figure S16. Crystal structure of $\left(\mathrm{P}_{6} \mathrm{ArC}_{2} \mathrm{Fe}_{2} \mathrm{H}\right.$ (5). Ellipsoids are shown at the $50 \%$ probability level. Hydrogen atoms and co-crystallized solvent molecules are not shown for clarity.

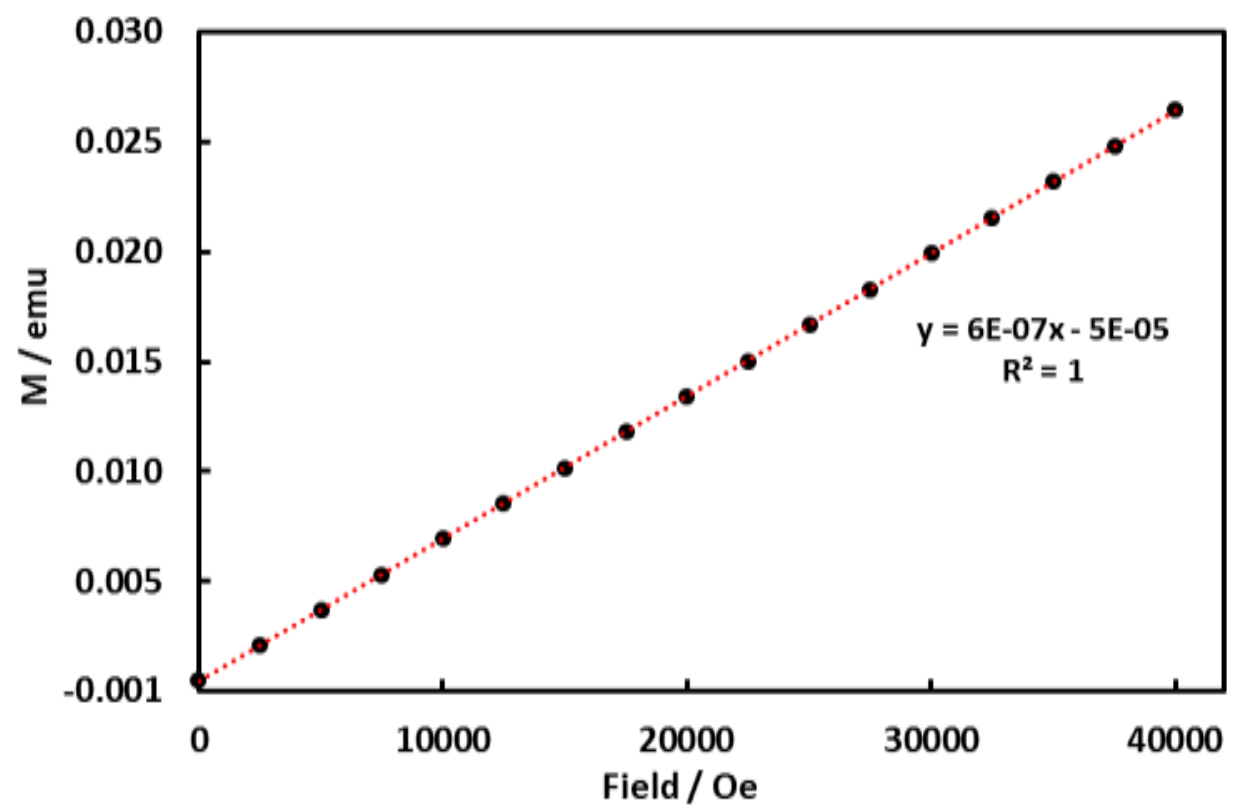

Figure S17. Magnetization data collected at $100 \mathrm{~K}$ from 0 to $4 \mathrm{~T}$ for $\left(\mathrm{P}_{6} \mathrm{ArC}\right) \mathrm{Fe}_{2} \mathrm{H}$ (5) to confirm the absence of ferromagnetic impurities. 


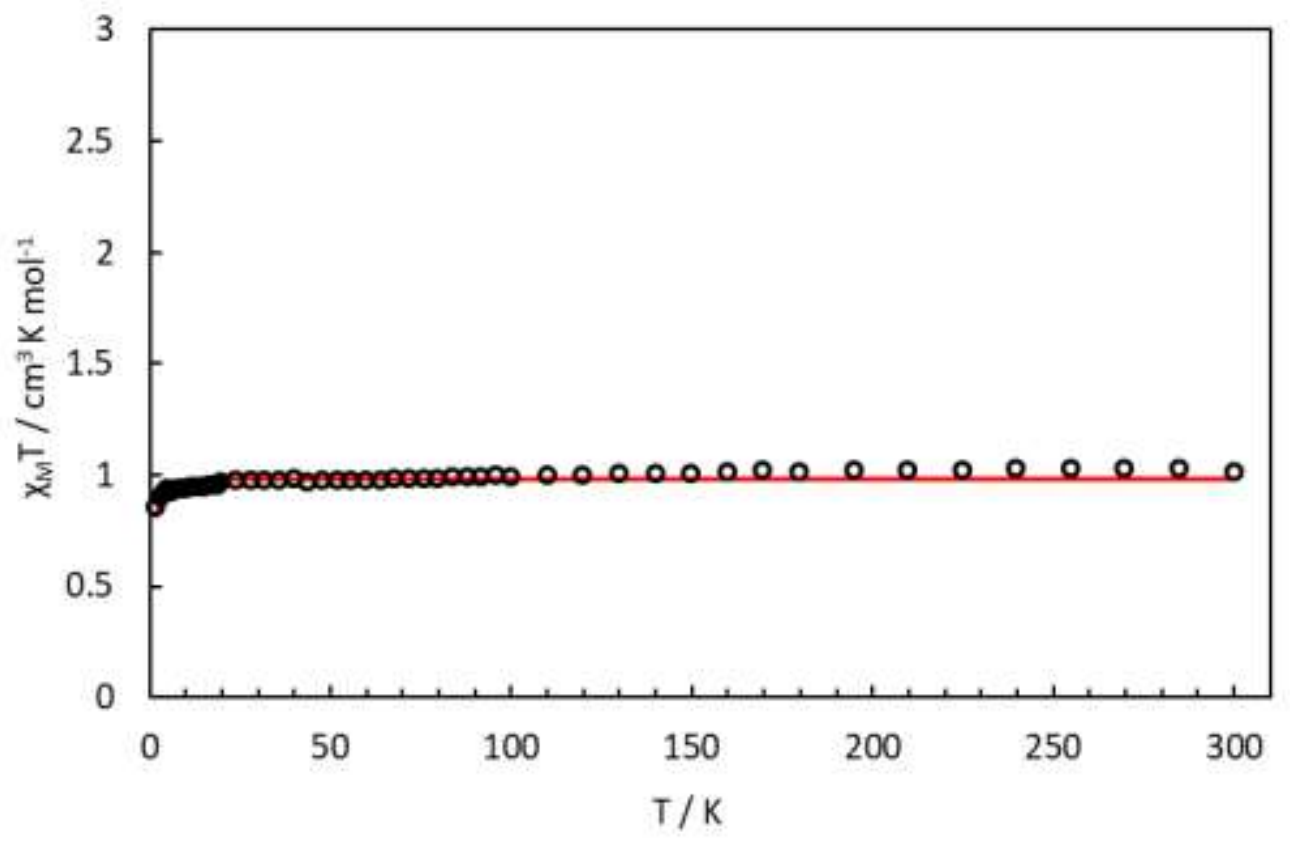

Figure S18. Direct current variable temperature magnetic susceptibility measurements for $\left(\mathrm{P}_{6} \mathrm{ArC}_{2} \mathrm{Fe}_{2} \mathrm{H}(\mathbf{5})\right.$ collected between 1.8 and $300 \mathrm{~K}$ with a $0.4 \mathrm{~T}$ field after diamagnetic correction (black circles). Fit parameters: $S=1, g=1.95, D=-3.78 \mathrm{~cm}^{-1}$ or $2.21 \mathrm{~cm}^{-1}$.

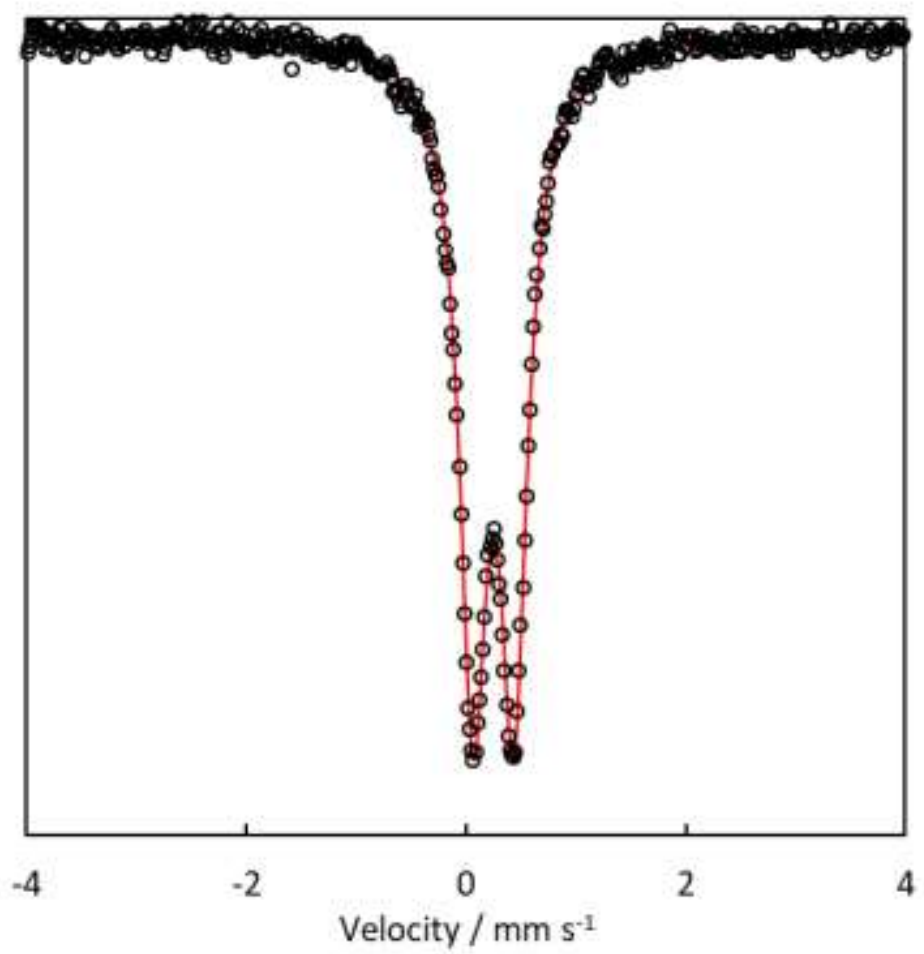

Figure S19. Solid-state ${ }^{57} \mathrm{Fe}$ Mössbauer spectrum of $\left(\mathrm{P}_{6} \mathrm{ArC}\right) \mathrm{Fe}_{2}(\mu-\mathrm{H})(\mathbf{5})$ collected at $80 \mathrm{~K}$ with a $50 \mathrm{mT}$ field applied perpendicular to the $\gamma$ rays. Data presented in black points, simulation represented by solid red line. Fit with $\delta=0.25 \mathrm{~mm} \mathrm{~s}^{-1},\left|\Delta E_{\mathrm{Q}}\right|=0.37 \mathrm{~mm} \mathrm{~s}^{-1}, \Gamma=0.30 \mathrm{~mm} \mathrm{~s}^{-1}$. 


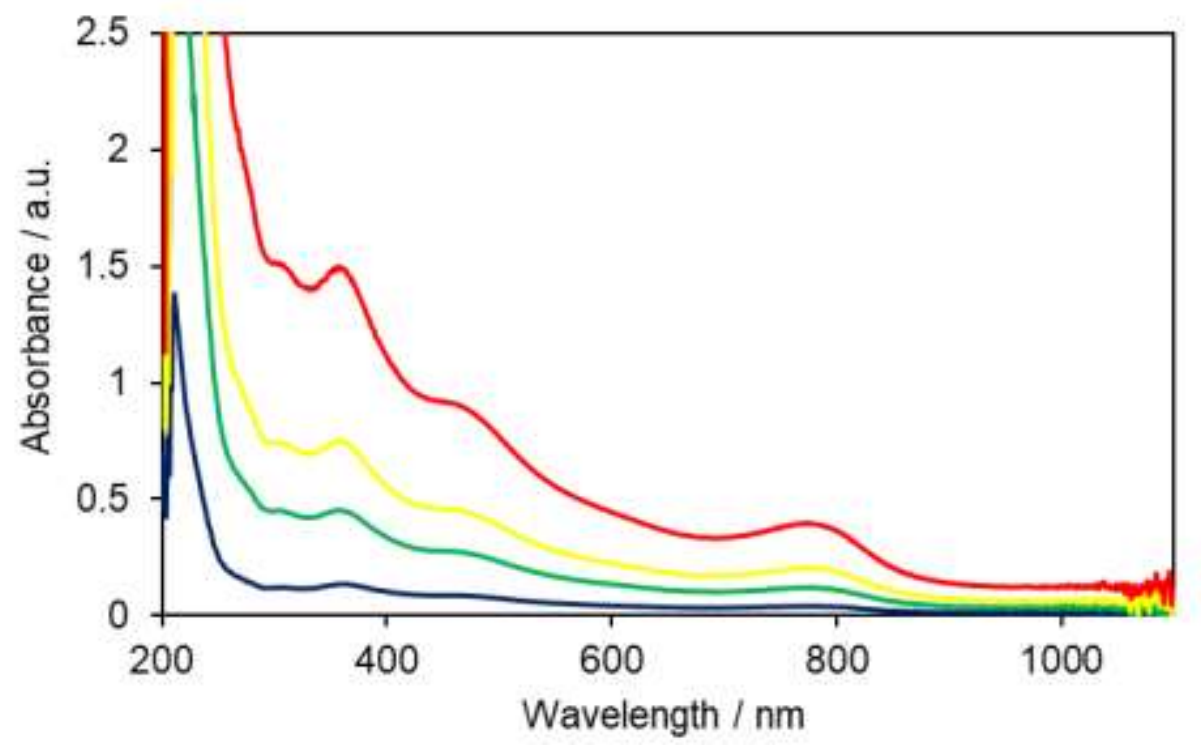

Figure S20. UV-Vis spectra of $\left(\mathrm{P}_{6} \mathrm{ArC}_{2} \mathrm{Fe}_{2}(\mu-\mathrm{H})(\mathbf{5})\right.$ collected in THF at concentrations of $10 \mu \mathrm{M}$ (blue), $30 \mu \mathrm{M}$ (green), $50 \mu \mathrm{M}$ (yellow) and $100 \mu \mathrm{M}$ (red).

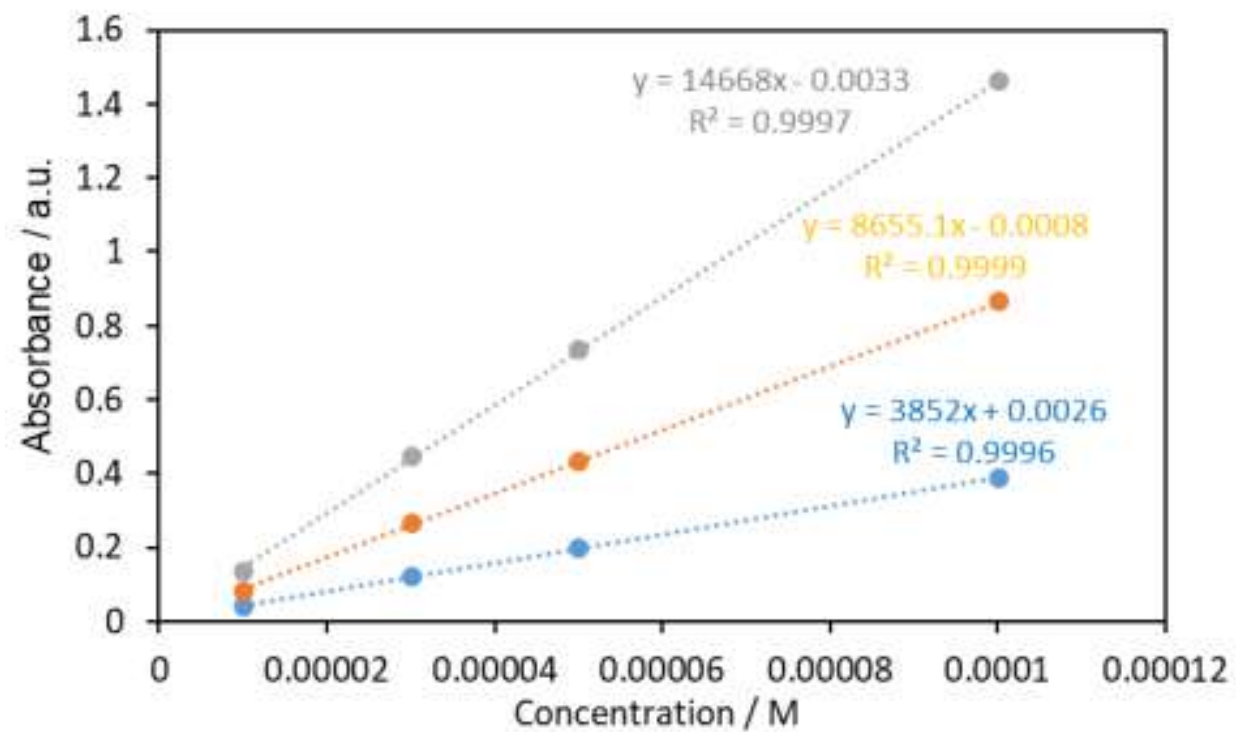

Figure S21. Beer's Law Plot for $\left(\mathrm{P}_{6} \mathrm{ArC}_{2} \mathrm{Fe}_{2}(\mu-\mathrm{H})(5)\right.$ at $366 \mathrm{~nm}$ (grey), $479 \mathrm{~nm}$ (orange), and 788 nm (blue). 

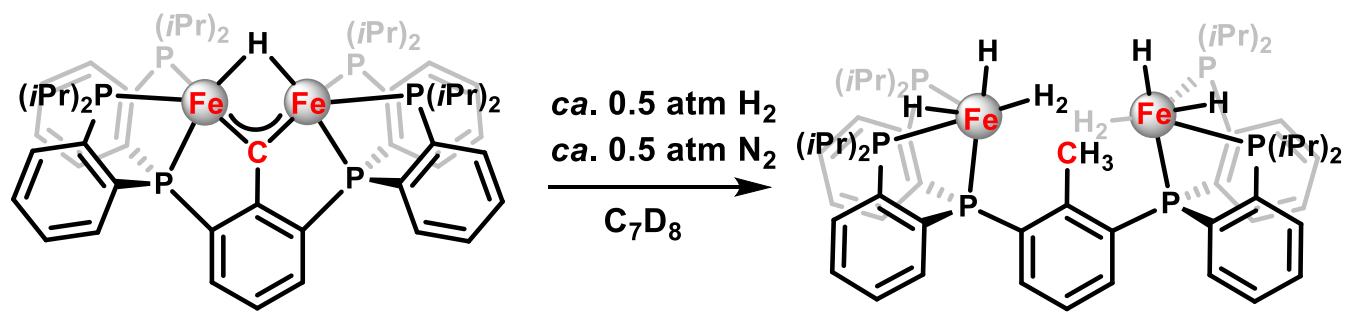

$\left(\mathbf{P}_{6} \mathrm{ArCH}_{3}\right) \mathrm{Fe}_{2}\left(\mathrm{H}_{2}\right)_{2}\left(\mathrm{H}_{4}, \mathbf{( 6 - \mathrm { H } _ { 2 } )}\right.$. A J Young NMR tube was charged with $\mathbf{5}$ (12.5 mg, $\left.0.012 \mathrm{mmol}\right)$ and dissolved in $\mathrm{C}_{7} \mathrm{D}_{8}(0.45 \mathrm{~mL})$. The tube was degassed on the Schlenk line with three freezepump-thaw cycles. A mixture of $\mathrm{H}_{2} / \mathrm{N}_{2}$ (c.a.50:50) was admitted to the tube at room temperature. This was rotated slowly until ${ }^{1} \mathrm{H}$ and ${ }^{31} \mathrm{P}$ NMR measurements indicated full consumption of 5, generally 7-8 days. Characterization of 6-H2 was conducted on samples prepared in situ, as exposure to a $\mathrm{N}_{2}$ atmosphere converts $\mathbf{6 - \mathbf { H } _ { 2 }}$ irreversibly (at room temperature in ambient light) to 6-N2. ${ }^{1} \mathrm{H}$ NMR $\left(400 \mathrm{MHz}, \mathrm{C}_{7} \mathrm{D}_{8}\right) \delta=7.60(\mathrm{t}, J=8 \mathrm{~Hz}, 4 \mathrm{H}), 7.39(\mathrm{~d}, J=8 \mathrm{~Hz}, 4 \mathrm{H}), 7.12(\mathrm{~s}, 2 \mathrm{H})$, 6.95-7.03 (m, 6H), $6.74(\mathrm{t}, J=8 \mathrm{~Hz}, 2 \mathrm{H}), 6.54(\mathrm{t}, J=8 \mathrm{~Hz}, 1 \mathrm{H}), 2.40(\mathrm{~m}, 4 \mathrm{H}), 2.05(\mathrm{~s}, 3 \mathrm{H}), 1.91$ $(\mathrm{m}, 4 \mathrm{H}), 1.32(\mathrm{dd}, J=8 \mathrm{~Hz}, 16 \mathrm{~Hz}, 12 \mathrm{H}), 1.09$ (dd, $J=8 \mathrm{~Hz}, 12 \mathrm{~Hz}, 12 \mathrm{H}), 0.97$ (dd, $J=8 \mathrm{~Hz}, 12$ $\mathrm{Hz}, 12 \mathrm{H}), 0.62(\mathrm{dd}, J=8 \mathrm{~Hz}, 12 \mathrm{~Hz}, 12 \mathrm{H}),-12.92(\mathrm{t}, J=28 \mathrm{~Hz}, 8 \mathrm{H}) .{ }^{31} \mathrm{P}\left\{{ }^{1} \mathrm{H}\right\} \mathrm{NMR}(162 \mathrm{MHz}$, $\left.\mathrm{C}_{6} \mathrm{D}_{6}\right) \delta=124.91$ (broad d, $\left.J=15 \mathrm{~Hz}, 4 \mathrm{P}\right), 110.00$ (t, $\left.J=19 \mathrm{~Hz}, 2 \mathrm{P}\right)$.

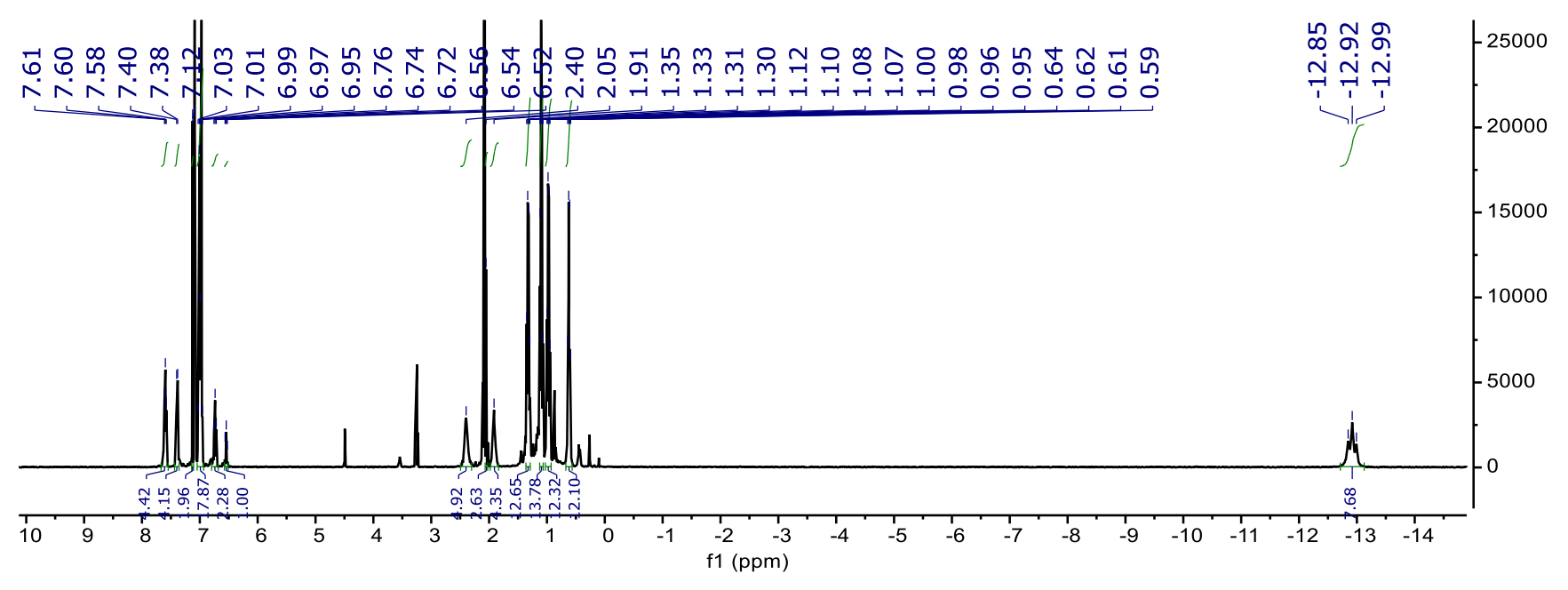

Figure S22. ${ }^{1} \mathrm{H}$ NMR $(400 \mathrm{MHz})$ of $\left(\mathrm{P}_{6} \mathrm{ArCH}_{3}\right) \mathrm{Fe}_{2}\left(\mathrm{H}_{2}\right)_{2}(\mathrm{H})_{4} \mathbf{( 6 - \mathbf { H } _ { 2 } )}$ in $\mathrm{C}_{7} \mathrm{D}_{8}$ 

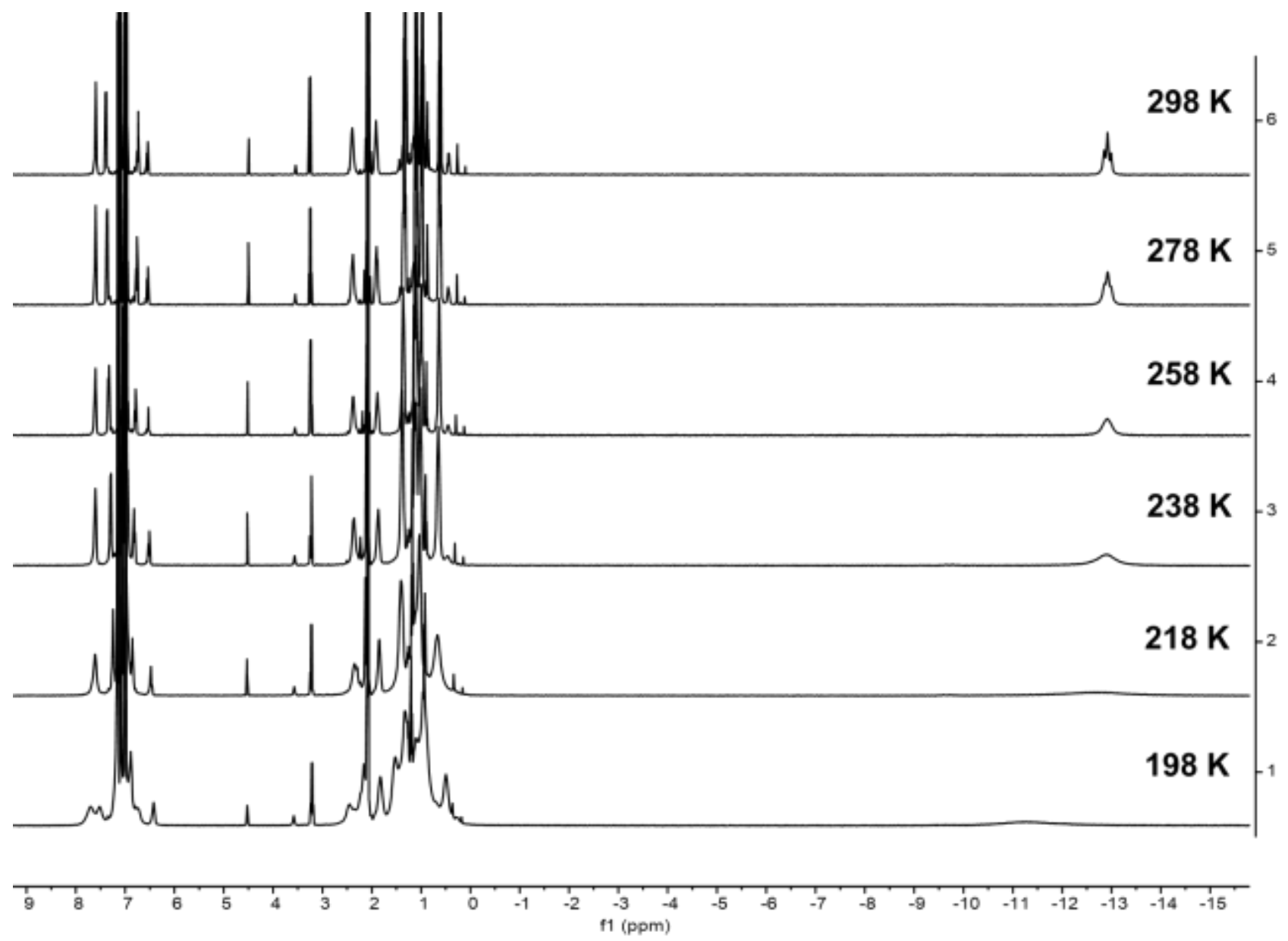

Figure S23. Variable Temperature ${ }^{1} \mathrm{H}$ NMR (400 MHz) of $\left(\mathrm{P}_{6} \mathrm{ArCH}_{3}\right) \mathrm{Fe}_{2}\left(\mathrm{H}_{2}\right)_{2}(\mathrm{H})_{4}\left(\mathbf{6}-\mathrm{H}_{2}\right)$ in $\mathrm{C}_{7} \mathrm{D}_{8}$ 

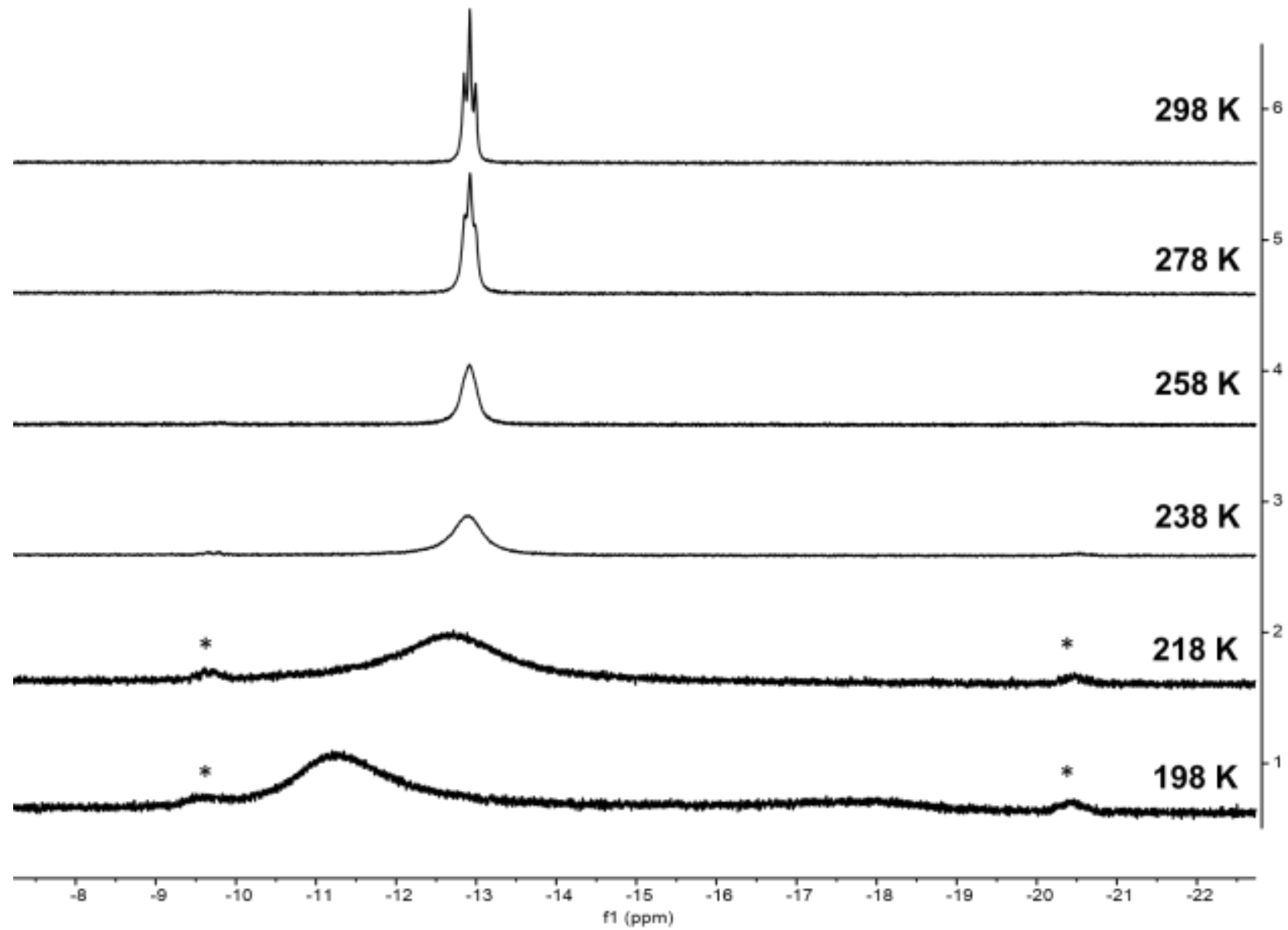

Figure S24. Variable Temperature ${ }^{1} \mathrm{H}$ NMR $(400 \mathrm{MHz})$ of $\left(\mathrm{P}_{6} \mathrm{ArCH}_{3}\right) \mathrm{Fe}_{2}\left(\mathrm{H}_{2}\right)_{2}(\mathrm{H})_{4}\left(\mathbf{6}-\mathrm{H}_{2}\right)$ in $\mathrm{C}_{7} \mathrm{D}_{8}$ focusing on hydridic region. Asterisks denote resonances associated with the hydride ligands of 6$\mathbf{H}_{2}$ which are partially resolved from exchange with the $\mathrm{H}_{2}$ ligands upon cooling.

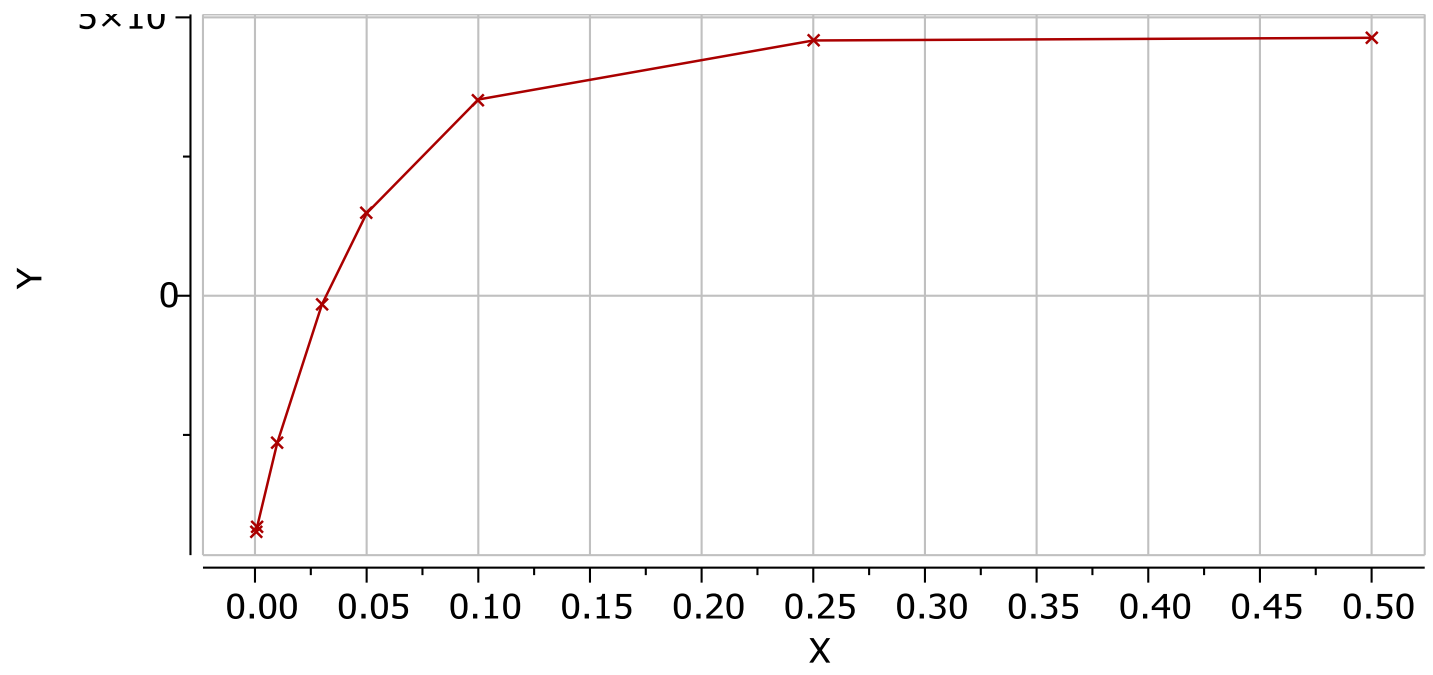

Figure S25. $\mathrm{T}_{1}$ Measurement for hydridic/dihydrogen resonance at $-12.92 \mathrm{ppm}$ in ${ }^{1} \mathrm{H}$ NMR spectrum of $\left(\mathrm{P}_{6} \mathrm{ArCH}_{3}\right) \mathrm{Fe}_{2}\left(\mathrm{H}_{2}\right)_{2}(\mathrm{H})_{4}\left(\mathbf{6}-\mathrm{H}_{2}\right)$ in $\mathrm{C}_{7} \mathrm{D}_{8}$ at $298 \mathrm{~K}$. Experimental data was fit to a three parameter exponential model $\left(\mathrm{A}+\mathrm{Be}^{-\mathrm{Cx}}\right)$ with $\mathrm{A}=463360, \mathrm{~B}=-896279$ and $\mathrm{C}=20.8969$. This yields a $\mathrm{T}_{1}$ of $48 \mathrm{~ms}$. 


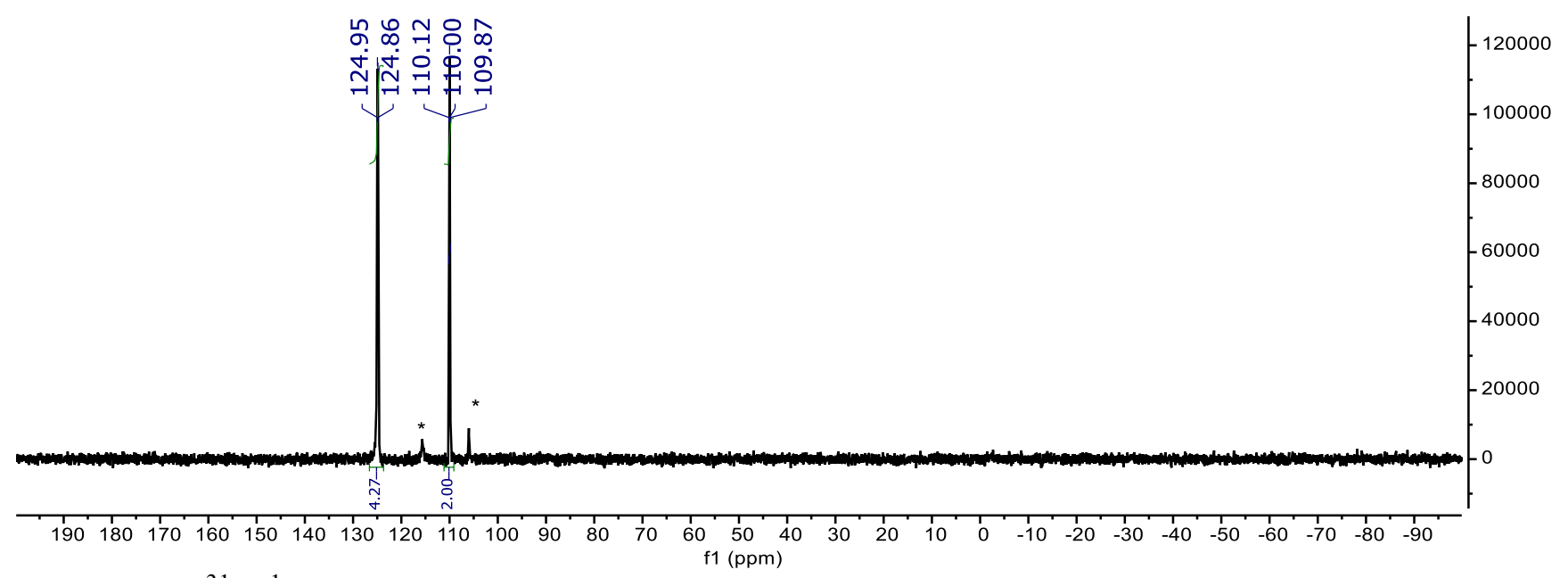

Figure S26. ${ }^{31} \mathrm{P}\left\{{ }^{1} \mathrm{H}\right\} \mathrm{NMR}(162 \mathrm{MHz})$ of $\left(\mathrm{P}_{6} \mathrm{ArCH}_{3}\right) \mathrm{Fe}_{2}\left(\mathrm{H}_{2}\right)_{2}(\mathrm{H})_{4}\left(\mathbf{6}-\mathrm{H}_{2}\right)$ in $\mathrm{C}_{7} \mathrm{D}_{8}$. Asterisks denote ${ }^{31} \mathrm{P}$ resonances associated with $\left(\mathrm{P}_{6} \mathrm{ArCH}_{3}\right) \mathrm{Fe}_{2}\left(\mathrm{~N}_{2}\right)_{2}(\mathrm{H})_{4}\left(\mathbf{6}-\mathrm{N}_{2}\right)$.
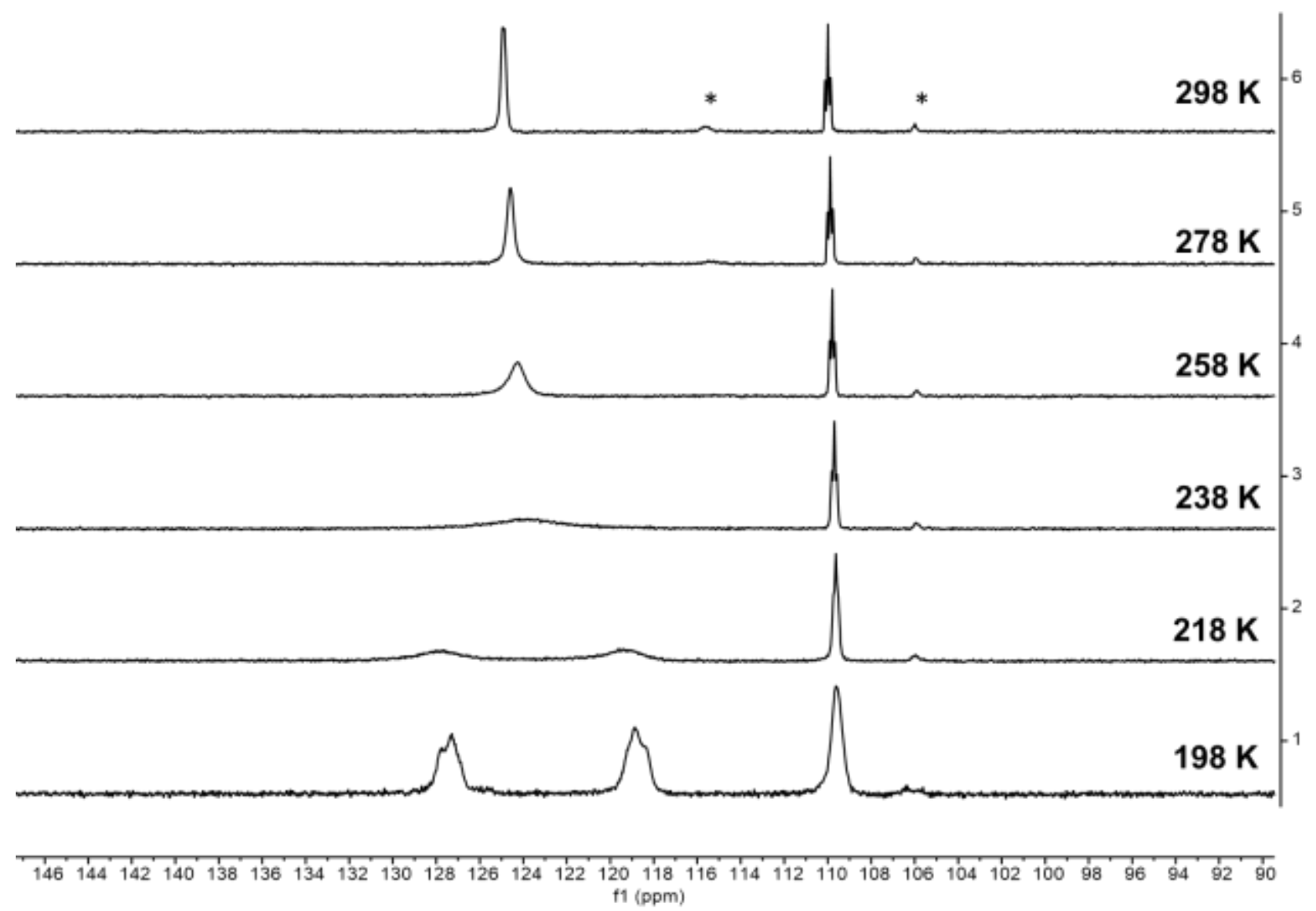

Figure S27. Variable Temperature ${ }^{31} \mathrm{P} \mathrm{NMR}(400 \mathrm{MHz})$ of $\left(\mathrm{P}_{6} \mathrm{ArCH}_{3}\right) \mathrm{Fe}_{2}\left(\mathrm{H}_{2}\right)_{2}(\mathrm{H})_{4}\left(\mathbf{6}-\mathrm{H}_{2}\right)$ in $\mathrm{C}_{7} \mathrm{D}_{8}$. 


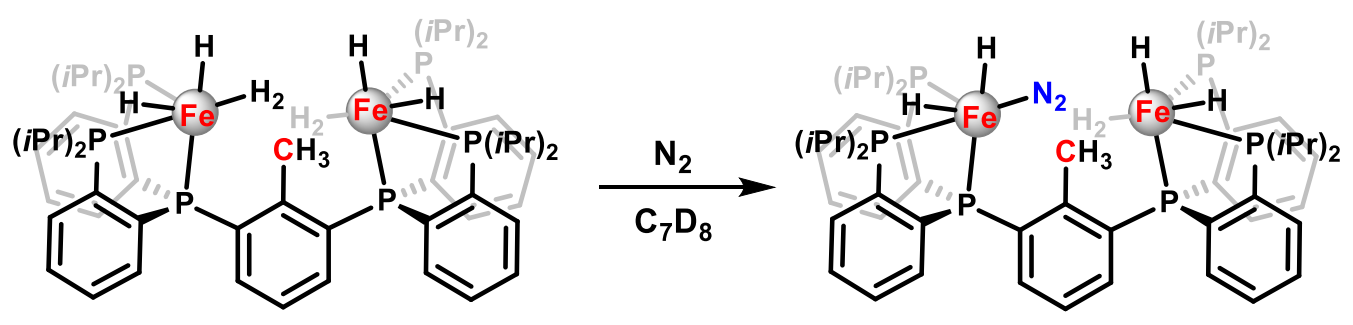

$\left(\mathbf{P}_{6} \mathbf{A r C H}_{3}\right) \mathbf{F e}_{2}\left(\mathbf{N}_{2}\right)_{2}(\mathbf{H})_{4},\left(6-\mathbf{N}_{2}\right)$. A J Young NMR tube was charged with 5 (12.5 mg, $\left.0.012 \mathrm{mmol}\right)$ and dissolved in $\mathrm{C}_{6} \mathrm{D}_{6}(0.45 \mathrm{~mL})$. The tube was degassed on the Schlenk line with three freezepump-thaw cycles. A mixture of $\mathrm{H}_{2} / \mathrm{N}_{2}$ (c.a.50:50) was admitted to the tube at room temperature. This was rotated slowly until ${ }^{1} \mathrm{H}$ NMR measurements indicated full consumption of 5, generally 7-8 days. At this point, the tube was once again degassed via three freeze-pump-thaw cycles and an atmosphere of $\mathrm{N}_{2}$ was admitted. After mixing for 24 hours, this process was repeated, resulting in complete conversion to 6 as indicated by ${ }^{1} \mathrm{H}$ and ${ }^{31} \mathrm{P}$ NMR spectroscopy. Yellow crystals suitable for X-ray diffraction were obtained by slow evaporation of a solution of $\mathbf{6}$ in tetrahydrofuran/hexamethyldisiloxane (1:1). ${ }^{1} \mathrm{H}$ NMR $\left(400 \mathrm{MHz}, \mathrm{C}_{7} \mathrm{D}_{8}\right) \delta=7.77(\mathrm{dd}, J=4 \mathrm{~Hz}, 12$ $\mathrm{Hz}, 4 \mathrm{H}), 7.51$ (broad d, $J=4 \mathrm{~Hz}, 4 \mathrm{H}), 7.27(\mathrm{~s}, 2 \mathrm{H}), 7.24(\mathrm{~m}, 6 \mathrm{H}), 6.97$ (t, $J=8 \mathrm{~Hz}, 2 \mathrm{H}), 6.78(\mathrm{t}, J$ $=8 \mathrm{~Hz}, 1 \mathrm{H}), 2.56(\mathrm{~m}, 8 \mathrm{H}), 2.30(\mathrm{~s}, 3 \mathrm{H}), 1.51(\mathrm{dd}, J=8 \mathrm{~Hz}, 16 \mathrm{~Hz}, 12 \mathrm{H}), 1.36(\mathrm{dd}, J=8 \mathrm{~Hz}, 12$ $\mathrm{Hz}, 12 \mathrm{H}), 1.31$ (dd, $J=8 \mathrm{~Hz}, 12 \mathrm{~Hz}, 12 \mathrm{H}), 0.59$ (dd, $J=8 \mathrm{~Hz}, 12 \mathrm{~Hz}, 12 \mathrm{H}),-9.75(\mathrm{~m}, 2 \mathrm{H}),-20.47$ $(\mathrm{m}, 2 \mathrm{H}) .{ }^{31} \mathrm{P}\left\{{ }^{1} \mathrm{H}\right\} \mathrm{NMR}\left(162 \mathrm{MHz}, \mathrm{C}_{6} \mathrm{D}_{6}\right) \delta=115.60($ broad d, 4P), 105.30 (broad t, $J=13 \mathrm{~Hz}$, 2P). IR (thin film from benzene): $2073 \mathrm{~cm}^{-1}\left(v_{\mathrm{N}-\mathrm{N}}\right), 1790 \mathrm{~cm}^{-1}\left(v_{\mathrm{Fe}-\mathrm{H}}\right)$.

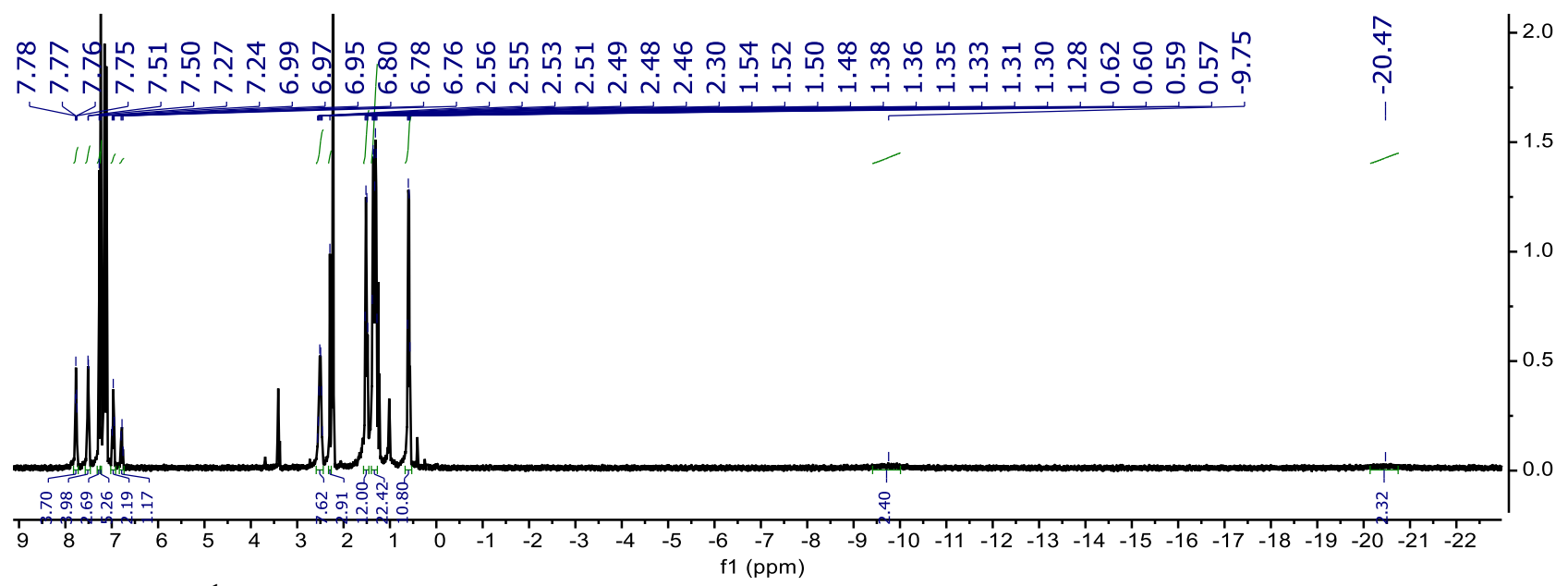

Figure S28. ${ }^{1} \mathrm{H}$ NMR (400 MHz) of $\left(\mathrm{P}_{6} \mathrm{ArCH}_{3}\right) \mathrm{Fe}_{2}\left(\mathrm{~N}_{2}\right)_{2}(\mathrm{H})_{4}\left(\mathbf{6}-\mathrm{N}_{2}\right)$ in $\mathrm{C}_{7} \mathrm{D}_{8}$ 

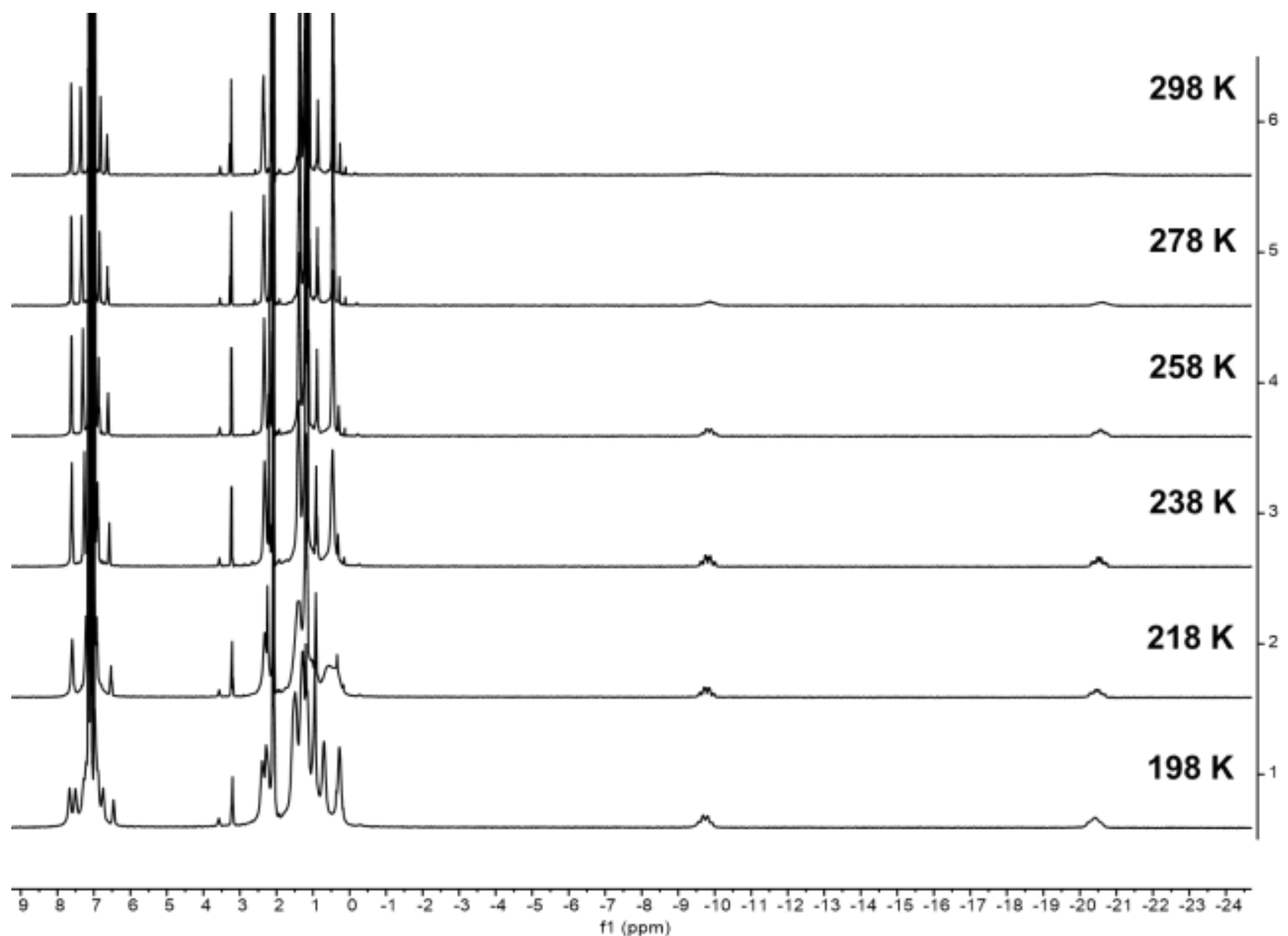

Figure S29. Variable Temperature ${ }^{1} \mathrm{H}$ NMR $(400 \mathrm{MHz})$ of $\left(\mathrm{P}_{6} \mathrm{ArCH}_{3}\right) \mathrm{Fe}_{2}\left(\mathrm{~N}_{2}\right)_{2}(\mathrm{H})_{4}\left(\mathbf{6}-\mathrm{N}_{2}\right)$ in $\mathrm{C}_{7} \mathrm{D}_{8}$ 


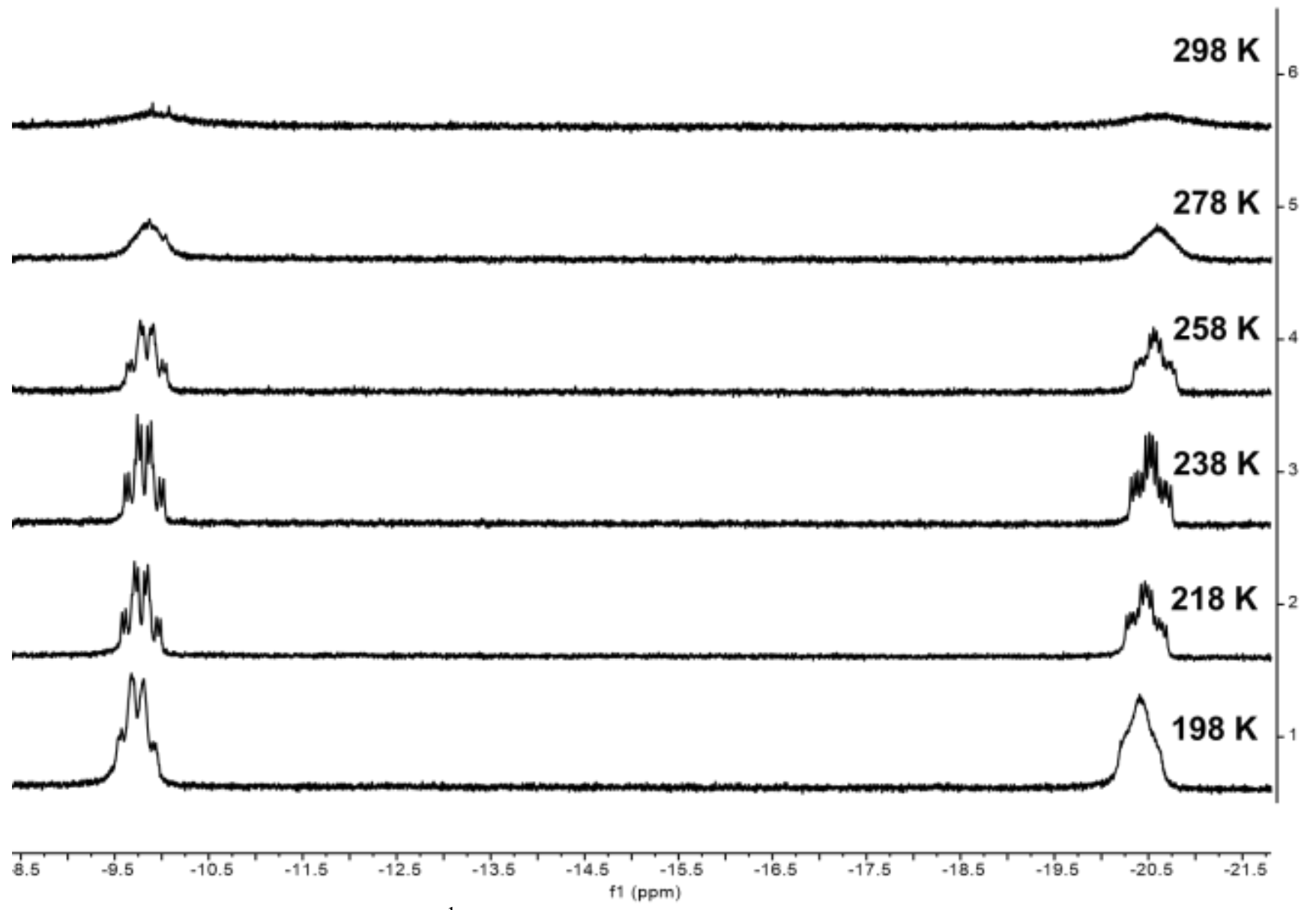

Figure S30. Variable Temperature ${ }^{1} \mathrm{H}$ NMR $(400 \mathrm{MHz})$ of $\left(\mathrm{P}_{6} \mathrm{ArCH}_{3}\right) \mathrm{Fe}_{2}\left(\mathrm{~N}_{2}\right)_{2}(\mathrm{H})_{4}\left(\mathbf{6}-\mathrm{N}_{2}\right)$ in $\mathrm{C}_{7} \mathrm{D}_{8}$ focusing on hydridic region. 


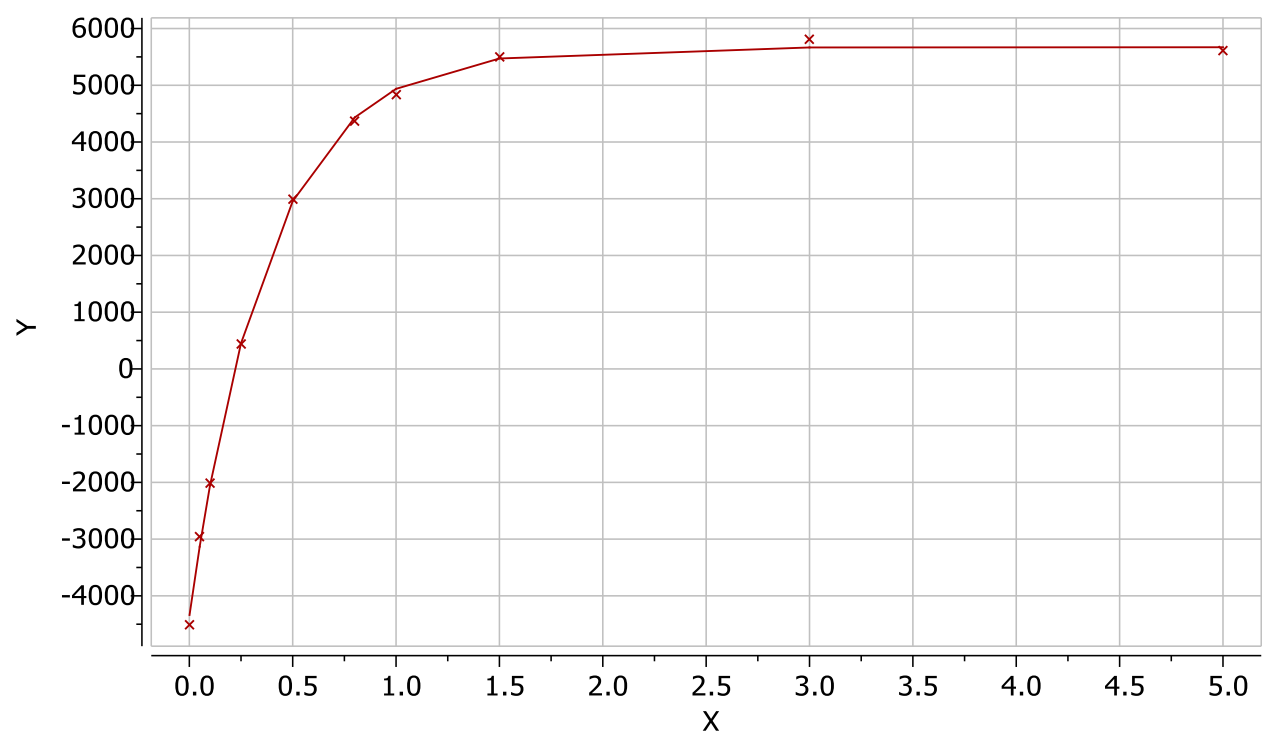

Figure S31. $\mathrm{T}_{1}$ Measurement for hydridic resonance at $-9.75 \mathrm{ppm}$ in ${ }^{1} \mathrm{H}$ NMR spectrum of $\left(\mathrm{P}_{6} \mathrm{ArCH}_{3}\right) \mathrm{Fe}_{2}\left(\mathrm{~N}_{2}\right)_{2}(\mathrm{H})_{4}\left(\mathbf{6}-\mathrm{N}_{2}\right)$ in $\mathrm{C}_{7} \mathrm{D}_{8}$ at $298 \mathrm{~K}$. Experimental data was fit to a three parameter exponential model $\left(\mathrm{A}+\mathrm{Be}^{-\mathrm{Cx}}\right)$ with $\mathrm{A}=5670.45, \mathrm{~B}=-10034$ and $\mathrm{C}=2.61569$. This yields a $\mathrm{T}_{1}$ of $382 \mathrm{~ms}$.

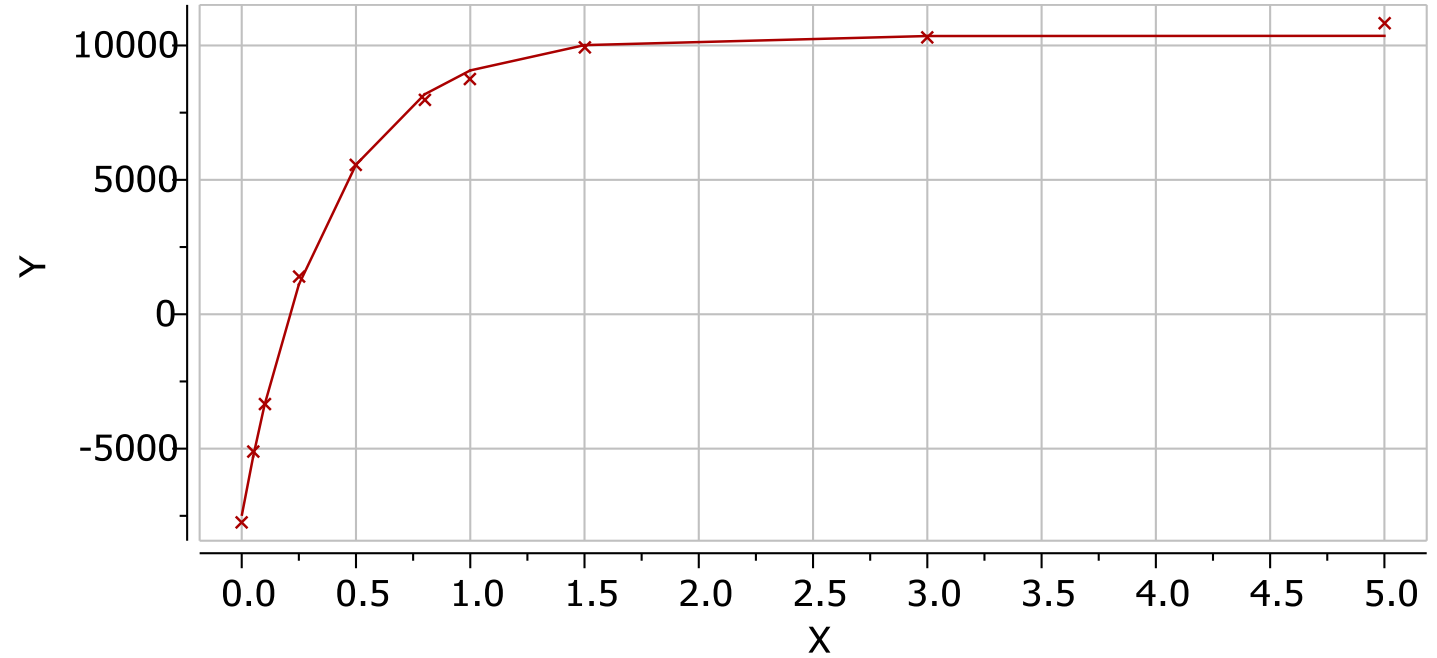

Figure S32. $\mathrm{T}_{1}$ Measurement for hydridic resonance at $-20.47 \mathrm{ppm}$ in ${ }^{1} \mathrm{H}$ NMR spectrum of $\left(\mathrm{P}_{6} \mathrm{ArCH}_{3}\right) \mathrm{Fe}_{2}\left(\mathrm{~N}_{2}\right)_{2}(\mathrm{H})_{4}\left(\mathbf{6}-\mathrm{N}_{2}\right)$ in $\mathrm{C}_{7} \mathrm{D}_{8}$ at $298 \mathrm{~K}$. Experimental data was fit to a three parameter exponential model $\left(\mathrm{A}+\mathrm{Be}^{-\mathrm{Cx}}\right)$ with $\mathrm{A}=10357.6, \mathrm{~B}=-17858.9$ and $\mathrm{C}=2.63072$. This yields a $\mathrm{T}_{1}$ of $380 \mathrm{~ms}$. 


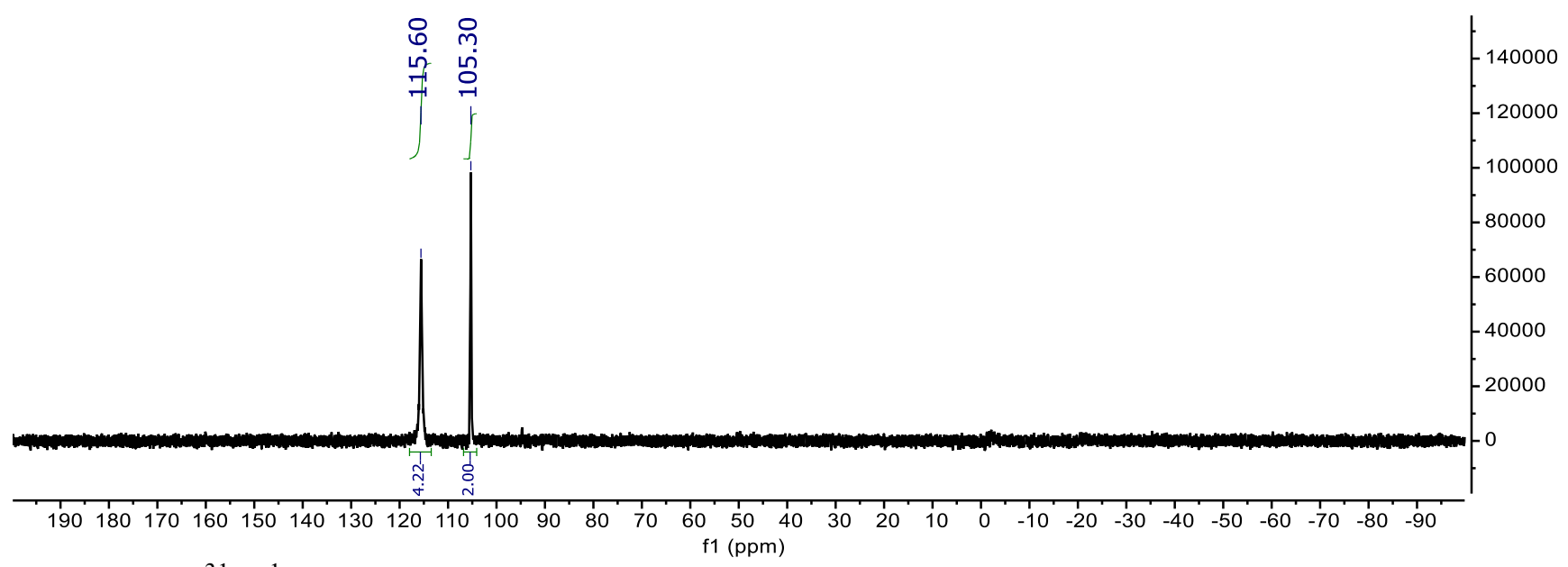

Figure S33. ${ }^{31} \mathrm{P}\left\{{ }^{1} \mathrm{H}\right\} \mathrm{NMR}(162 \mathrm{MHz})$ of $\left(\mathrm{P}_{6} \mathrm{ArCH}_{3}\right) \mathrm{Fe}_{2}\left(\mathrm{~N}_{2}\right)_{2}(\mathrm{H})_{4}\left(\mathbf{6}-\mathrm{N}_{2}\right)$ in $\mathrm{C}_{7} \mathrm{D}_{8}$.

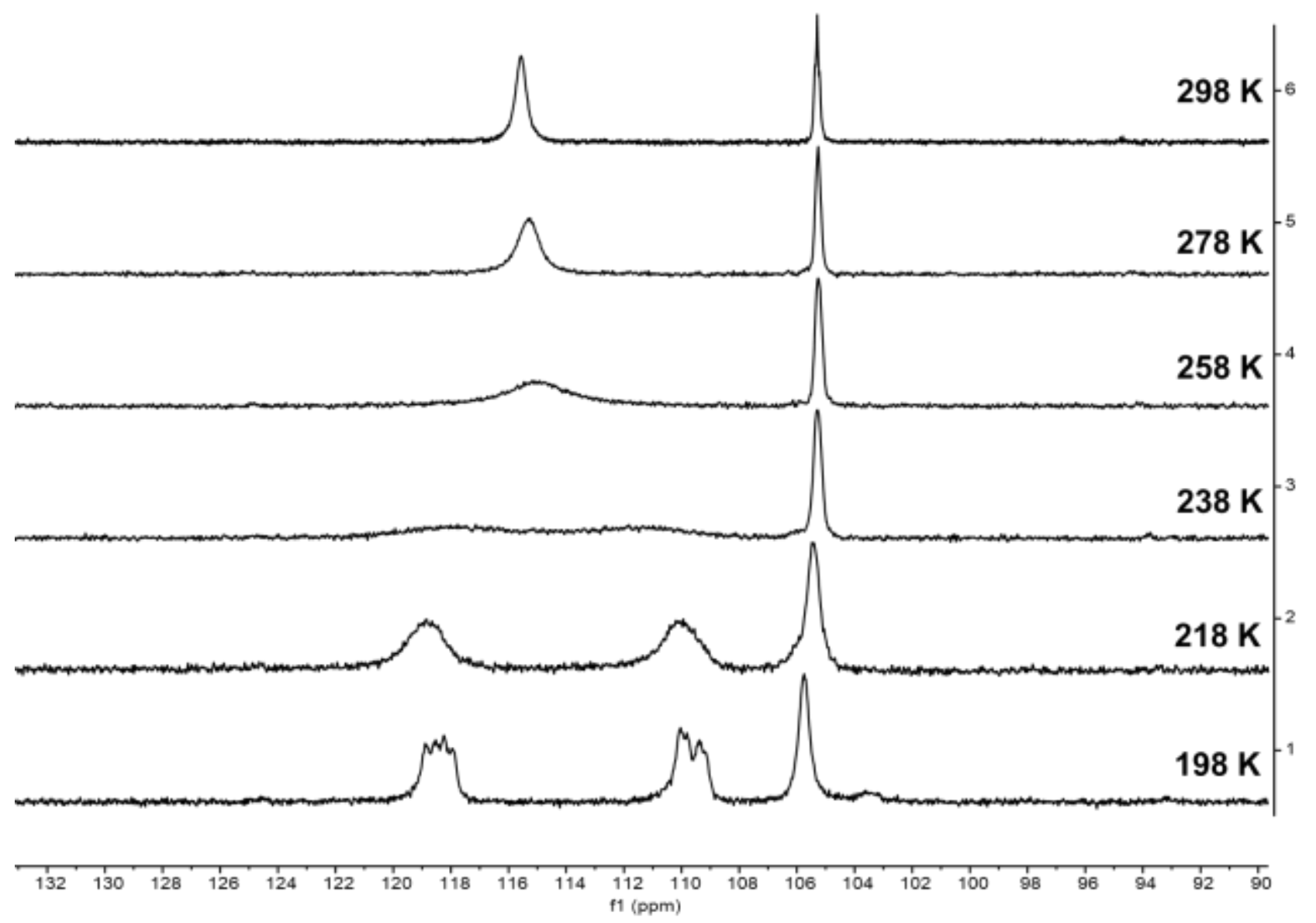

Figure S34. Variable Temperature ${ }^{31} \mathrm{P}\left\{{ }^{1} \mathrm{H}\right\}$ NMR $(162 \mathrm{MHz})$ of $\left(\mathrm{P}_{6} \mathrm{ArCH}_{3}\right) \mathrm{Fe}_{2}\left(\mathrm{~N}_{2}\right)_{2}(\mathrm{H})_{4}\left(\mathbf{6}-\mathrm{N}_{2}\right)$ in $\mathrm{C}_{7} \mathrm{D}_{8}$. 


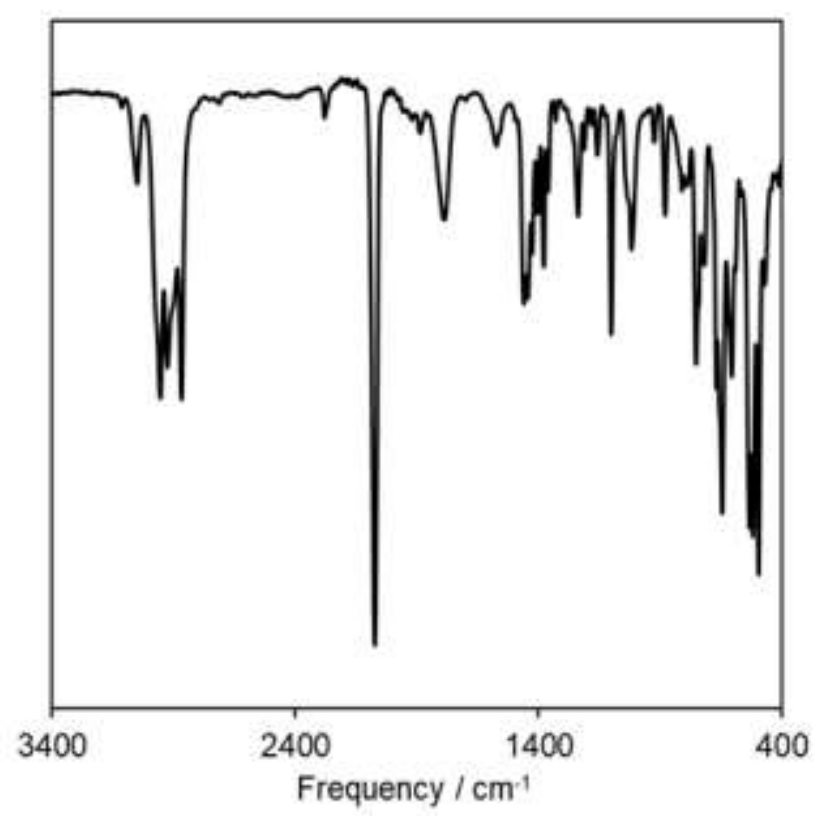

Figure S35. ATR-IR spectrum of $\left(\mathrm{P}_{6} \mathrm{ArCH}_{3}\right) \mathrm{Fe}_{2}\left(\mathrm{~N}_{2}\right)_{2}(\mathrm{H})_{4}\left(\mathbf{6}-\mathrm{N}_{2}\right)$. Characteristic $v_{\mathrm{NN}}$ feature is observed at $2073 \mathrm{~cm}^{-1}$. Characteristic $v_{\mathrm{FeH}}$ feature is observed at $1790 \mathrm{~cm}^{-1}$.

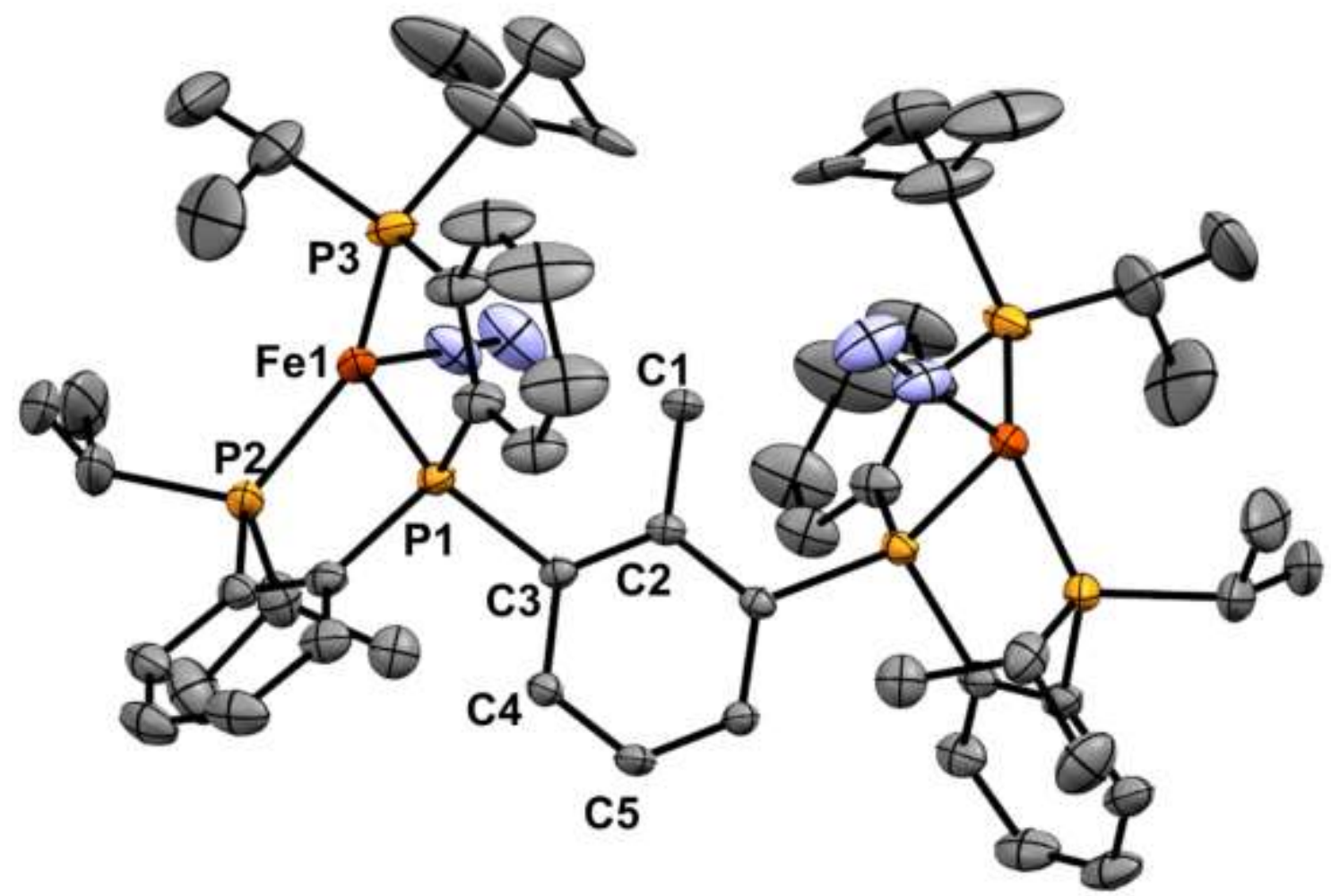

Figure S36. Crystal structure of $\left(\mathrm{P}_{6} \mathrm{ArCH}_{3}\right) \mathrm{Fe}_{2}\left(\mathrm{~N}_{2}\right)_{2}(\mathrm{H})_{4}\left(\mathbf{6}-\mathbf{N}_{2}\right)$. Ellipsoids are shown at the 50\% probability level. Hydrogen atoms and co-crystallized solvent molecules are not shown for clarity. 


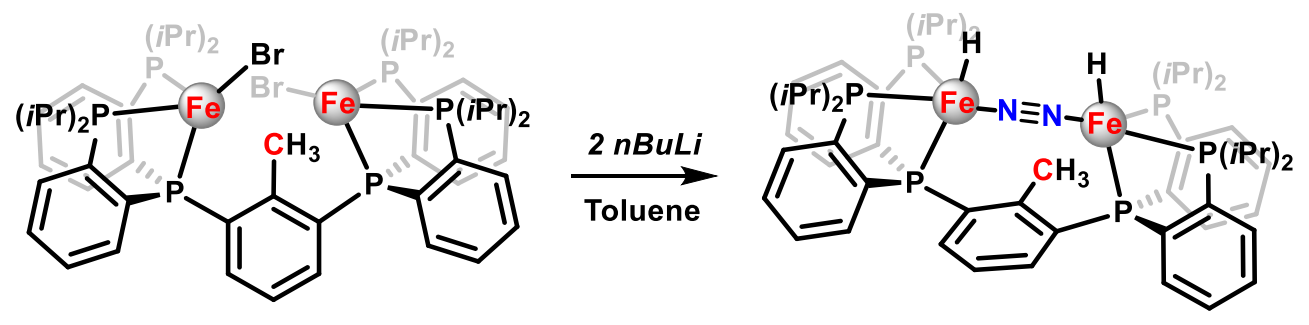

$\left[\mathrm{P}_{6} \mathbf{A r C H}_{3}\right](\mathbf{F e H})_{2}\left(\boldsymbol{\mu}-\mathbf{N}_{2}\right),(7)$. A suspension of $4(41.8 \mathrm{mg}, 0.035 \mathrm{mmol}, 1$ equiv.) in toluene (6 $\mathrm{mL}$ ) was chilled to $-78{ }^{\circ} \mathrm{C}$ in a glovebox cold well. A solution of $n \mathrm{BuLi}(43 \mu \mathrm{L}, 1.61 \mathrm{M}, 2$ equiv.) was added, resulting in a gradual darkening of the solution. After stirring in the cold well for four hours, the vial was warmed to room temperature, leading to an immediate color change to green. The mixture was then filtered over Celite and concentrated to dryness. The green residue was washed with pentane $(3 \times 1 \mathrm{~mL})$ and diethyl ether $(3 \times 1 \mathrm{~mL})$, affording 7 in spectroscopically pure form. Complex 7 is thermally sensitive, decomposing fully in solution over the course of 6-8 hours at room temperature. Crystals suitable for X-ray diffraction were obtained by cooling a dilute toluene/hexamethyldisiloxane solution of 7 to $-35{ }^{\circ} \mathrm{C} .{ }^{1} \mathrm{H}$ NMR $\left(400 \mathrm{MHz}, \mathrm{C}_{6} \mathrm{D}_{6}\right) \delta=8.31$ (broad m, 2H), 8.17 (broad m, 2H), 7.51 (dd, $J=8 \mathrm{~Hz}, 16 \mathrm{~Hz}, 4 \mathrm{H}), 6.89-7.07$ (m, 11H), 2.19 (septet, $J=$ $4 \mathrm{~Hz}, 4 \mathrm{H}), 1.86$ (m, 6H), 1.75 (septet, $J=4 \mathrm{~Hz}, 4 \mathrm{H}), 1.67$ (dd, $J=8 \mathrm{~Hz}, 16 \mathrm{~Hz}, 6 \mathrm{H}), 1.62$ (s, 3H), $1.51(\mathrm{~m}, 6 \mathrm{H}), 1.02$ (dd, $J=4 \mathrm{~Hz}, 16 \mathrm{~Hz}, 6 \mathrm{H}), 0.88(\mathrm{t}, J=8 \mathrm{~Hz}, 6 \mathrm{H}), 0.38$ (dd, $J=4 \mathrm{~Hz}, 16 \mathrm{~Hz}$, $6 \mathrm{H}),-0.08(\mathrm{dd}, J=4 \mathrm{~Hz}, 16 \mathrm{~Hz}, 6 \mathrm{H}),-0.36(\mathrm{dd}, 4 \mathrm{~Hz}, 12 \mathrm{~Hz}, 6 \mathrm{H}),-3.96(\mathrm{~b}, 2 \mathrm{H}) .{ }^{31} \mathrm{P}\left\{{ }^{1} \mathrm{H}\right\} \mathrm{NMR}$ $\left(162 \mathrm{MHz}, \mathrm{C}_{6} \mathrm{D}_{6}\right) \delta=126.37$ (b, 2P), $121.33(\mathrm{~d}, J=42 \mathrm{~Hz}, 2 \mathrm{P}), 110.83$ (broad dd, $J=19 \mathrm{~Hz}, 44$ $\mathrm{Hz}, 2 \mathrm{P})$.

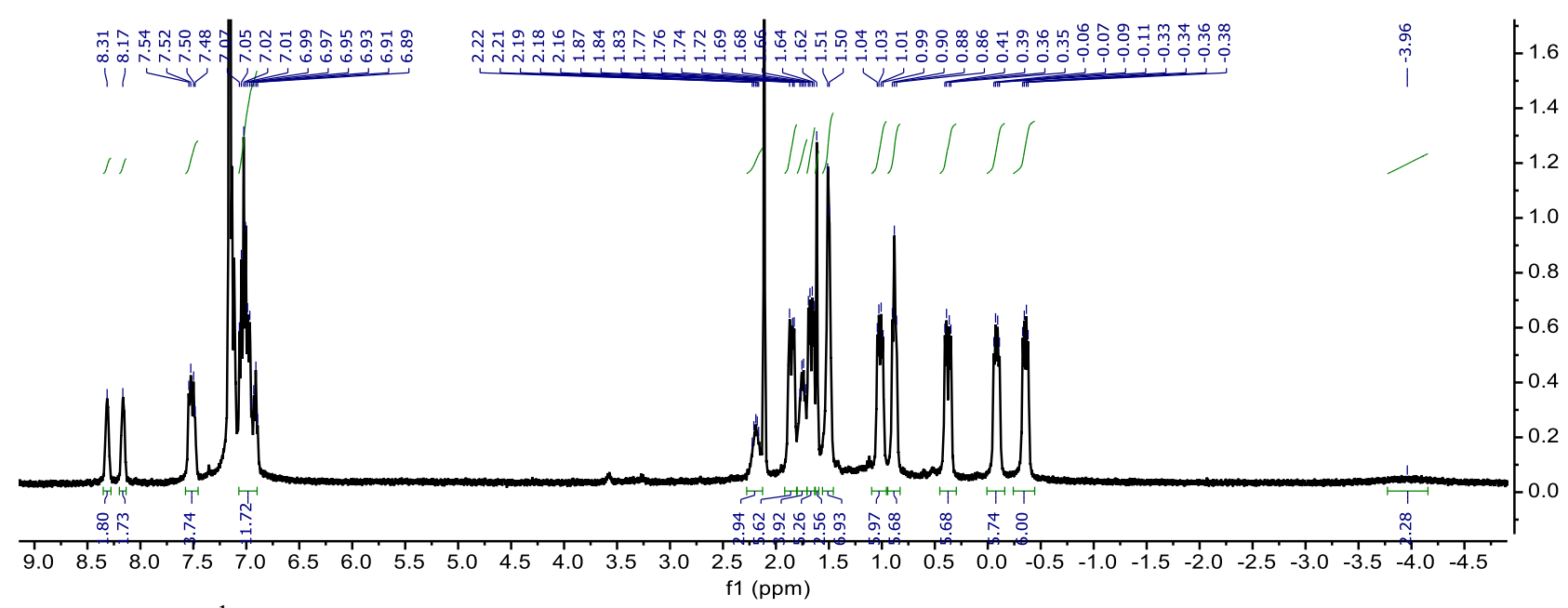

Figure S37. ${ }^{1} \mathrm{H}$ NMR $(400 \mathrm{MHz})$ of $\left[\mathrm{P}_{6} \mathrm{ArCH}_{3}\right](\mathrm{FeH})_{2}\left(\mu-\mathrm{N}_{2}\right)(7)$ in $\mathrm{C}_{6} \mathrm{D}_{6}$. 


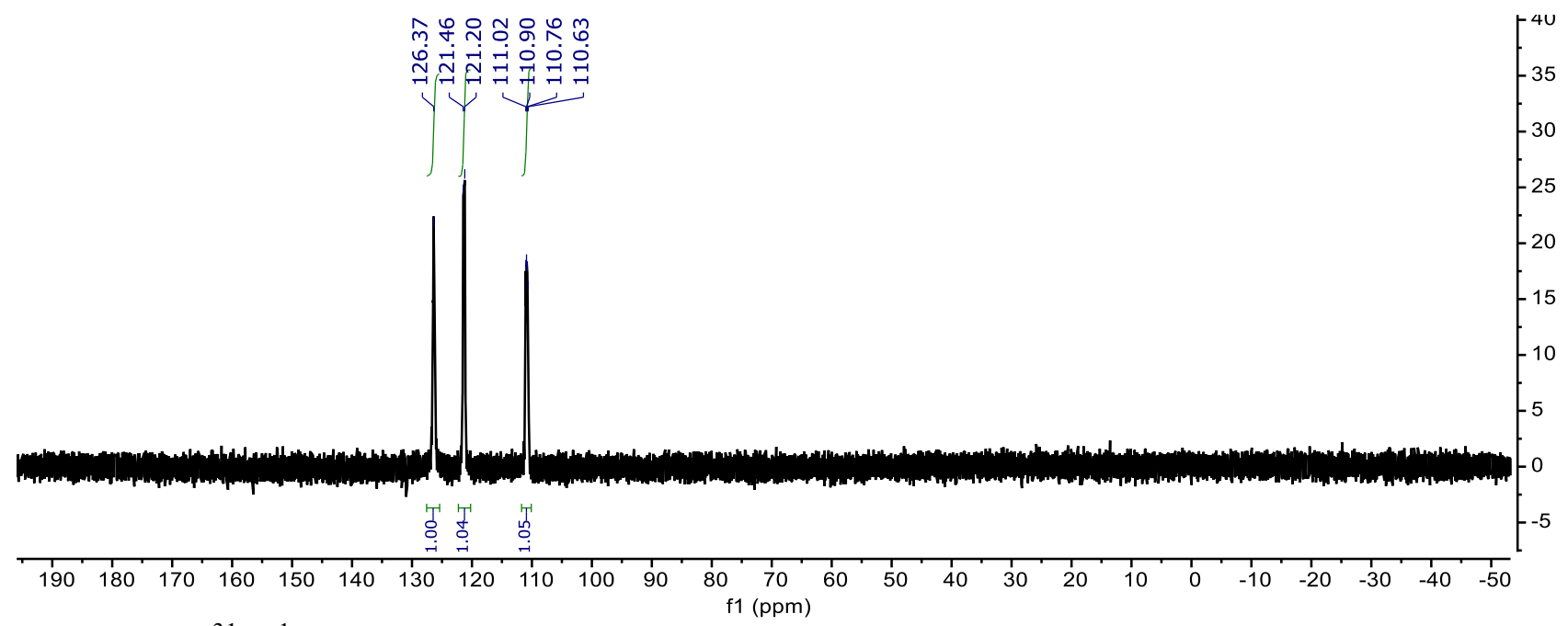

Figure S38. ${ }^{31} \mathrm{P}\left\{{ }^{1} \mathrm{H}\right\} \mathrm{NMR}(162 \mathrm{MHz})$ of $\left[\mathrm{P}_{6} \mathrm{ArCH}_{3}\right](\mathrm{FeH})_{2}\left(\mu-\mathrm{N}_{2}\right)(7)$ in $\mathrm{C}_{6} \mathrm{D}_{6}$.

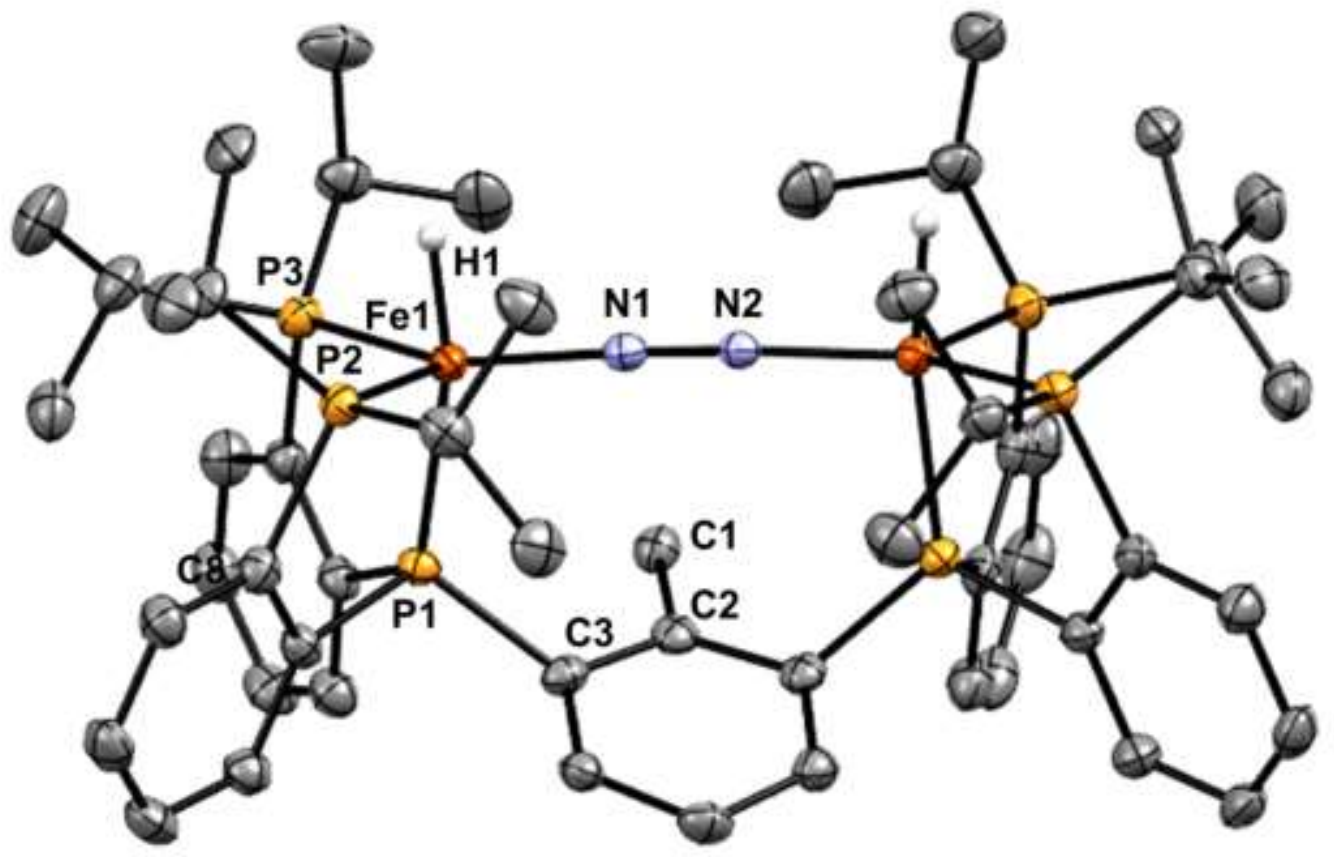

Figure S39. Crystal structure of $\left[\mathrm{P}_{6} \mathrm{ArCH}_{3}\right](\mathrm{FeH})_{2}\left(\mu-\mathrm{N}_{2}\right)(7)$. Ellipsoids are shown at the $50 \%$ probability level. Hydrogen atoms and co-crystallized solvent molecules are not shown for clarity. 


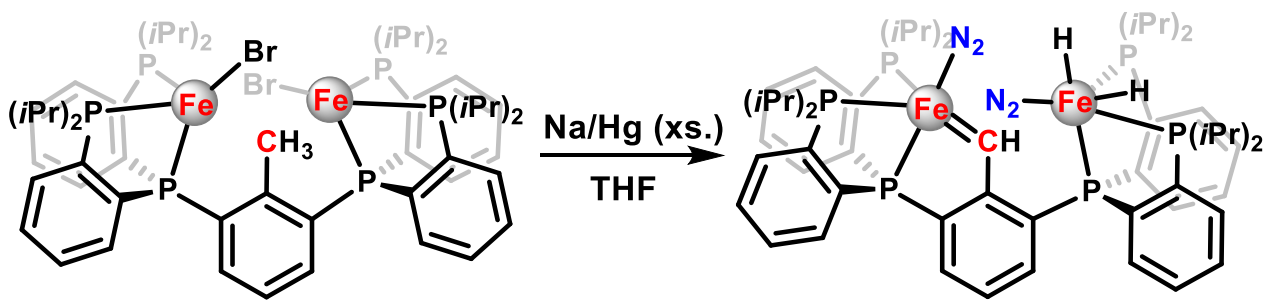

$\left(\mathrm{P}_{6} \mathrm{ArCH}_{\mathbf{C}} \mathrm{Fe}_{2}\left(\mathrm{~N}_{2}\right)(\mathbf{H})_{2},(\mathbf{8})\right.$. A $2 \%$ mixture of sodium amalgam was prepared by adding $\mathrm{Hg}$ via syringe to $\mathrm{Na}(25.9 \mathrm{mg}, 10$ equiv.) smeared along the side of a one dram vial. The sodium amalgam was poured into a thawing solution of 4 (134.6 $\mathrm{mg}, 0.112 \mathrm{mmol}, 1$ equiv.) in tetrahydrofuran (14 $\mathrm{mL}$ ). After stirring for an additional three hours under nitrogen atmosphere, the orange-brown solution was filtered over Celite and concentrated in vacuo. The residue was triturated twice with hexanes and then extracted with toluene. The filtrate was concentrated in vacuo and washed with pentane ( $3 \times 2 \mathrm{~mL}$ ), affording $8(100 \mathrm{mg}, 84 \%)$ in $>90 \%$ purity as a brown powder. Crystals suitable for X-ray diffraction were obtained by slow evaporation of a concentrated solution of $\mathbf{8}$ in pentane/diethyl ether $(1: 1) .{ }^{1} \mathrm{H}$ NMR $\left(400 \mathrm{MHz}, \mathrm{C}_{6} \mathrm{D}_{6}\right) \delta=11.03(\mathrm{~s}, 1 \mathrm{H}), 7.97(\mathrm{t}, J=8 \mathrm{~Hz}, 2 \mathrm{H})$, $7.58(\mathrm{t}, J=8 \mathrm{~Hz}, 2 \mathrm{H}), 7.50(\mathrm{~d}, J=8 \mathrm{~Hz}, 2 \mathrm{H}), 7.32(\mathrm{~m}, 2 \mathrm{H}), 6.92-7.02(\mathrm{~m}, 6 \mathrm{H}), 6.84(\mathrm{t}, J=8 \mathrm{~Hz}$, $4 \mathrm{H}), 6.70(\mathrm{t}, J=8 \mathrm{~Hz}, 1 \mathrm{H}), 2.83(\mathrm{~m}, 2 \mathrm{H}), 2.63(\mathrm{~m}, 2 \mathrm{H}), 2.12(\mathrm{~m}, 2 \mathrm{H}), 2.00(\mathrm{~m}, 2 \mathrm{H}), 1.55(\mathrm{~m}, 12 \mathrm{H})$, $1.41(\mathrm{~m}, 6 \mathrm{H}), 1.15(\mathrm{~m}, 6 \mathrm{H}), 0.97(\mathrm{~m}, 6 \mathrm{H}), 0.89(\mathrm{~m}, 6 \mathrm{H}), 0.51(\mathrm{~m}, 6 \mathrm{H}), 0.32(\mathrm{~m}, 6 \mathrm{H}),-9.74(\mathrm{~m}$, $1 \mathrm{H}),-20.65(\mathrm{~m}, 1 \mathrm{H}) .{ }^{31} \mathrm{P}\left\{{ }^{1} \mathrm{H}\right\} \mathrm{NMR}\left(162 \mathrm{MHz}, \mathrm{C}_{6} \mathrm{D}_{6}\right) \delta=126.1(\mathrm{t}, J=21 \mathrm{~Hz}, 1 \mathrm{P}), 114.7$ (broad $\mathrm{d}, J=45 \mathrm{~Hz}, 2 \mathrm{P}), 103.6(\mathrm{~b}, 1 \mathrm{P}), 93.8(\mathrm{~d}, J=21 \mathrm{~Hz}, 2 \mathrm{P}) .{ }^{13} \mathrm{C}\left\{{ }^{1} \mathrm{H}\right\} \mathrm{NMR}\left(101 \mathrm{MHz}, \mathrm{C}_{6} \mathrm{D}_{6}\right) \delta 224.62$ $-223.56(\mathrm{~m}), 167.10$ (d, $J=10.5 \mathrm{~Hz}), 166.57$ (d, $J=10.6 \mathrm{~Hz}), 150.85$ (dt, $J=38.6,19.3 \mathrm{~Hz}$ ), $149.67(\mathrm{dt}, J=45.0,16.4 \mathrm{~Hz}), 147.91-146.72(\mathrm{~m}), 145.78-143.86(\mathrm{~m}), 138.87(\mathrm{~d}, J=8.6 \mathrm{~Hz})$, $130.81-130.21(\mathrm{~m}), 129.73(\mathrm{~d}, J=16.9 \mathrm{~Hz}), 129.17-128.49(\mathrm{~m}), 123.41,65.56,34.09,31.32$, 30.49 (d, $J=3.1 \mathrm{~Hz}), 30.27-29.28(\mathrm{~m}), 24.46$ (td, $J=16.8,7.6 \mathrm{~Hz}), 22.37,20.43,20.01,19.54$ (dd, $J=8.5,4.3 \mathrm{~Hz}), 18.92(\mathrm{q}, J=3.6,3.0 \mathrm{~Hz}), 15.26,13.95$. IR (thin film from benzene): 2070 $\mathrm{cm}^{-1}\left(v_{\mathrm{N}-\mathrm{N}}\right), 2006 \mathrm{~cm}^{-1}\left(v_{\mathrm{N}-\mathrm{N}}\right), 1789 \mathrm{~cm}^{-1}\left(v_{\mathrm{Fe}-\mathrm{H}}\right)$

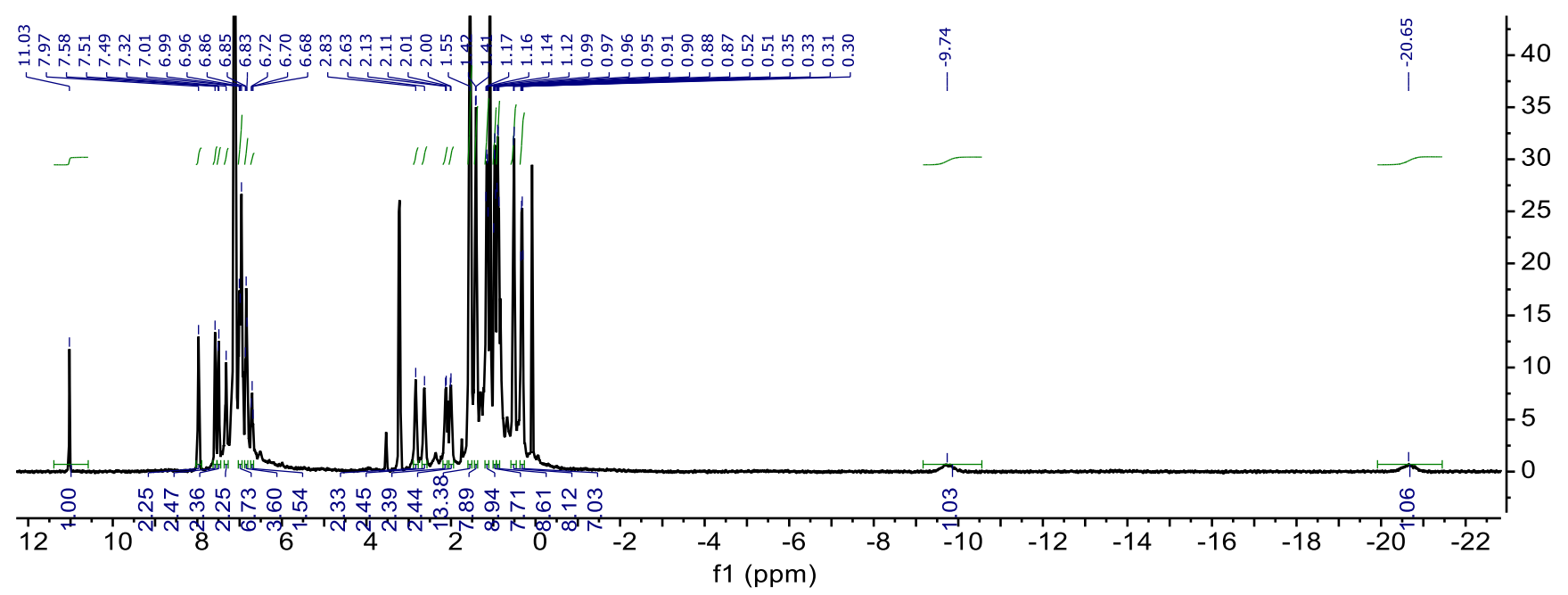

Figure S40. ${ }^{1} \mathrm{H}$ NMR $(400 \mathrm{MHz})$ of $\left(\mathrm{P}_{6} \mathrm{ArCH}\right) \mathrm{Fe}_{2}\left(\mathrm{~N}_{2}\right)_{2}(\mathrm{H})_{2}(\mathbf{8})$ in $\mathrm{C}_{6} \mathrm{D}_{6}$ 

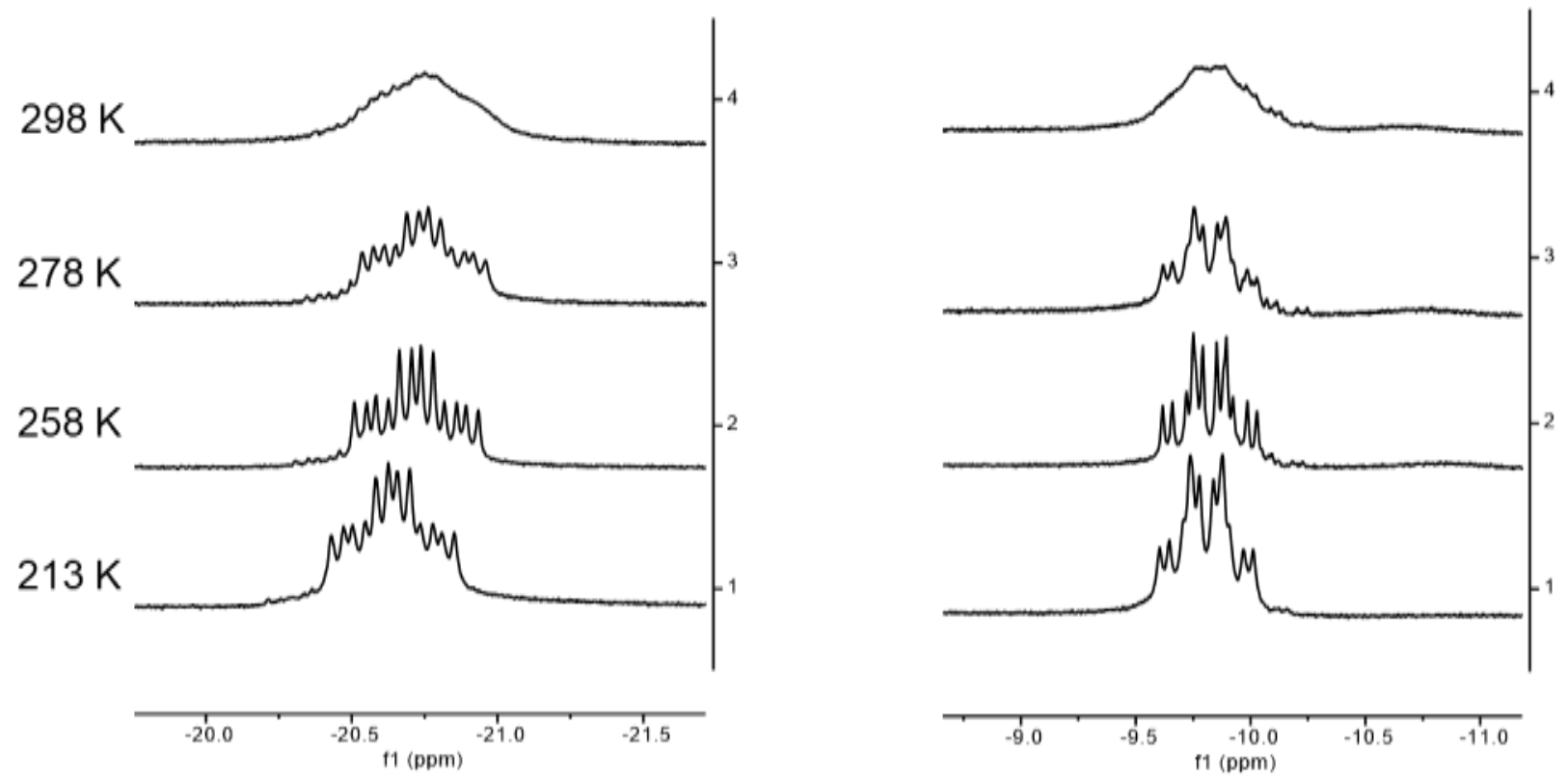

Figure S41. ${ }^{1} \mathrm{H}$ NMR $(400 \mathrm{MHz})$ of $\left(\mathrm{P}_{6} \mathrm{ArCH} \mathrm{Fe}_{2}\left(\mathrm{~N}_{2}\right)_{2}(\mathrm{H})_{2}(\mathbf{8})\right.$ in $\mathrm{C}_{7} \mathrm{D}_{8}$ at variable temperatures to resolve ${ }^{2} J_{\mathrm{HP}}$.

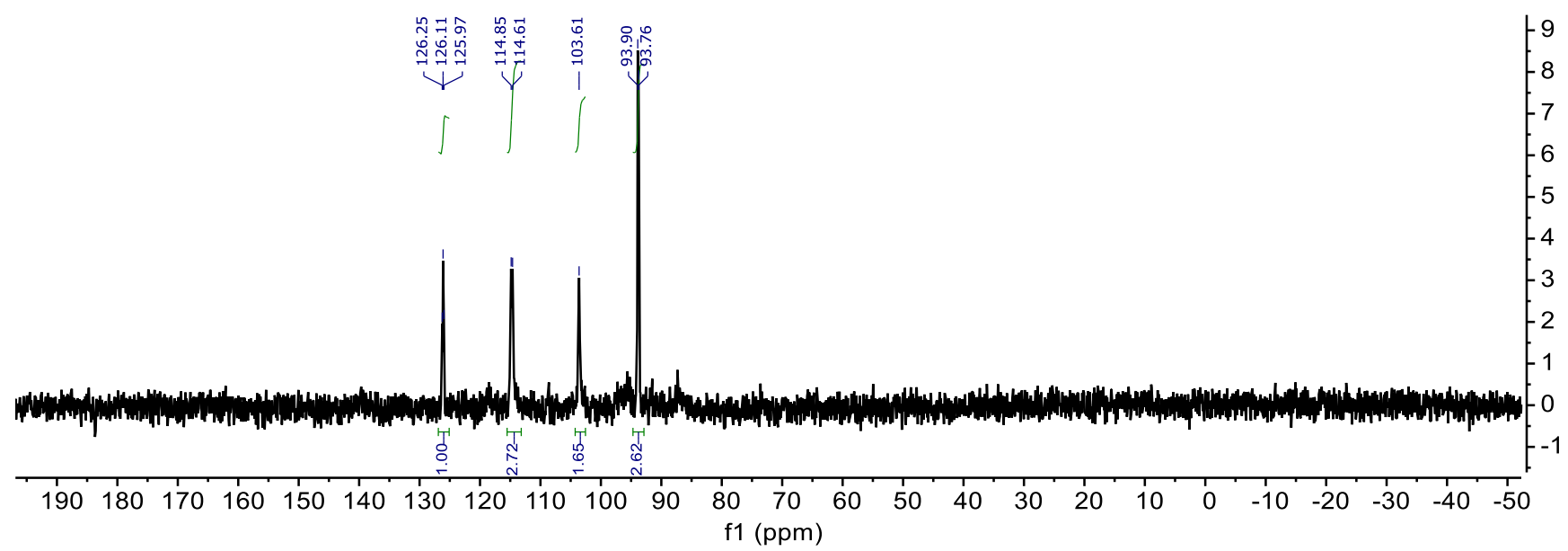

Figure S42. ${ }^{31} \mathrm{P}\left\{{ }^{1} \mathrm{H}\right\} \mathrm{NMR}(162 \mathrm{MHz})$ of $\left(\mathrm{P}_{6} \mathrm{ArCH}\right) \mathrm{Fe}_{2}\left(\mathrm{~N}_{2}\right)_{2}(\mathrm{H})_{2}(\mathbf{8})$ in $\mathrm{C}_{6} \mathrm{D}_{6}$ 


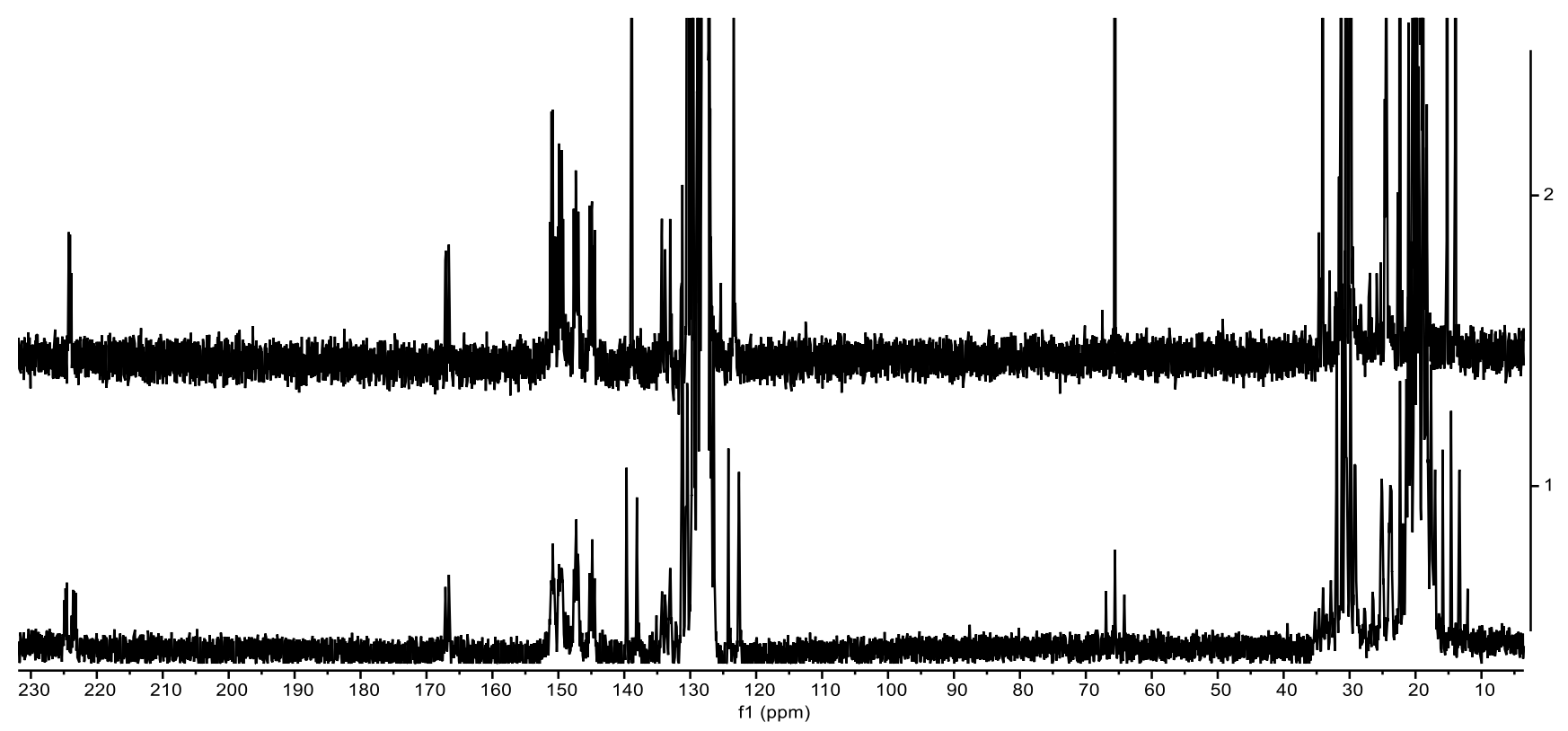

Figure S43. ${ }^{13} \mathrm{C}\left\{{ }^{1} \mathrm{H}\right\}$ NMR $(101 \mathrm{MHz})$ of $\left(\mathrm{P}_{6} \mathrm{ArCH} \mathrm{Fe}_{2}\left(\mathrm{~N}_{2}\right)_{2}(\mathrm{H})_{2}(\mathbf{8})\right.$ in $\mathrm{C}_{6} \mathrm{D}_{6}$ (top). Gate decoupled ${ }^{13} \mathrm{C}$ NMR (101 MHz) of $\left(\mathrm{P}_{6} \mathrm{ArCH} \mathrm{Fe}_{2}\left(\mathrm{~N}_{2}\right)_{2}(\mathrm{H})_{2}(\mathbf{8})\right.$ in $\mathrm{C}_{6} \mathrm{D}_{6}$ (Bottom).

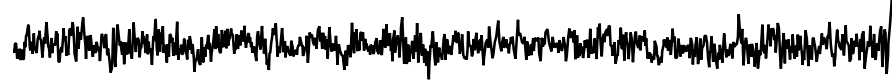
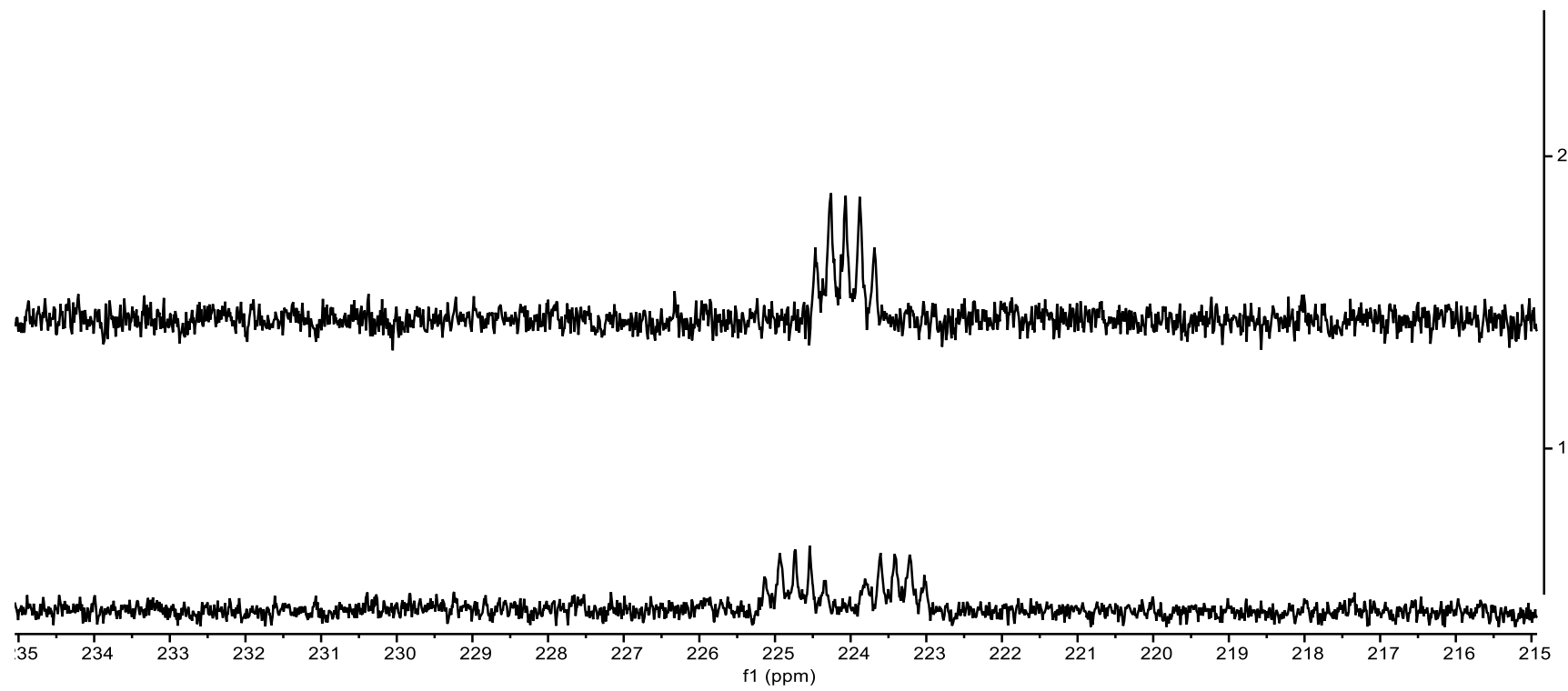

Figure S44. ${ }^{13} \mathrm{C}\left\{{ }^{1} \mathrm{H}\right\}$ NMR $(101 \mathrm{MHz})$ of $\left(\mathrm{P}_{6} \mathrm{ArCH}_{2} \mathrm{Fe}_{2}\left(\mathrm{~N}_{2}\right)_{2}(\mathrm{H})_{2}(\mathbf{8})\right.$ in $\mathrm{C}_{6} \mathrm{D}_{6}$ (top) focusing on the carbene resonance. Gate decoupled ${ }^{13} \mathrm{C}$ NMR $(101 \mathrm{MHz})$ of $\left(\mathrm{P}_{6} \mathrm{ArCH}\right) \mathrm{Fe}_{2}\left(\mathrm{~N}_{2}\right)_{2}(\mathrm{H})_{2}(\mathbf{8})$ in $\mathrm{C}_{6} \mathrm{D}_{6}$ (Bottom). From this data: $\delta=224 \mathrm{ppm},{ }^{1} J_{\mathrm{CH}}=134 \mathrm{~Hz},{ }^{2} J_{\mathrm{CP}}=20$ and $39 \mathrm{~Hz}$. 


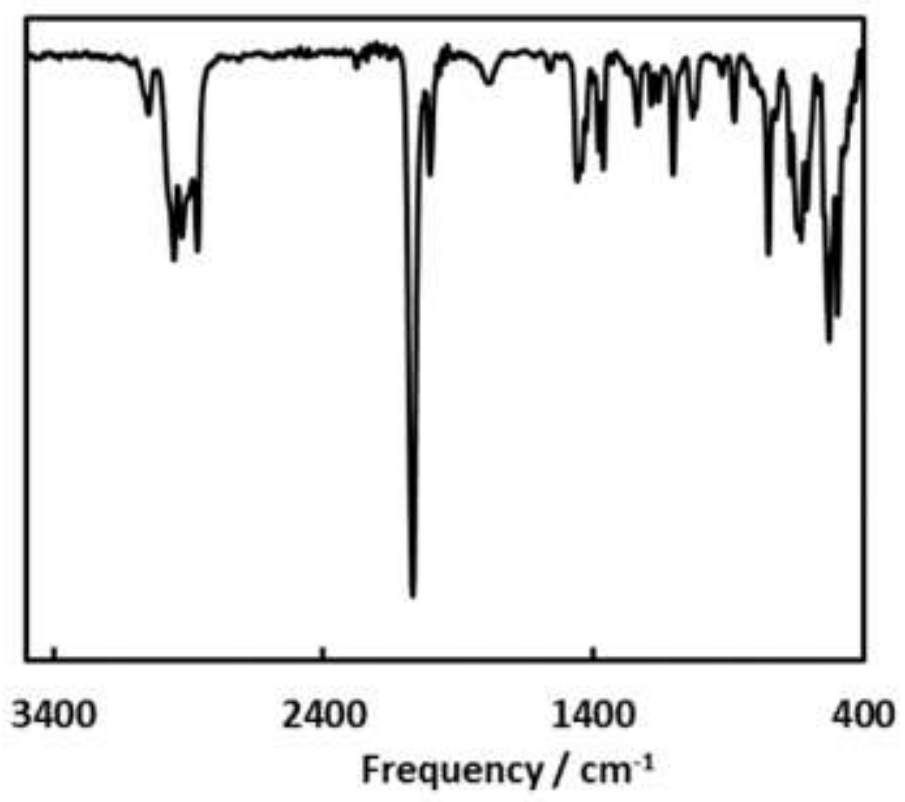

Figure S45. ATR-IR spectrum of $\left(\mathrm{P}_{6} \mathrm{ArCH}\right) \mathrm{Fe}_{2}\left(\mathrm{~N}_{2}\right)_{2}(\mathrm{H})_{2}(8)$. Characteristic $v_{\mathrm{NN}}$ features are observed at 2006 and $2070 \mathrm{~cm}^{-1}$. Characteristic $v_{\mathrm{FeH}}$ feature is observed at $1789 \mathrm{~cm}^{-1}$.

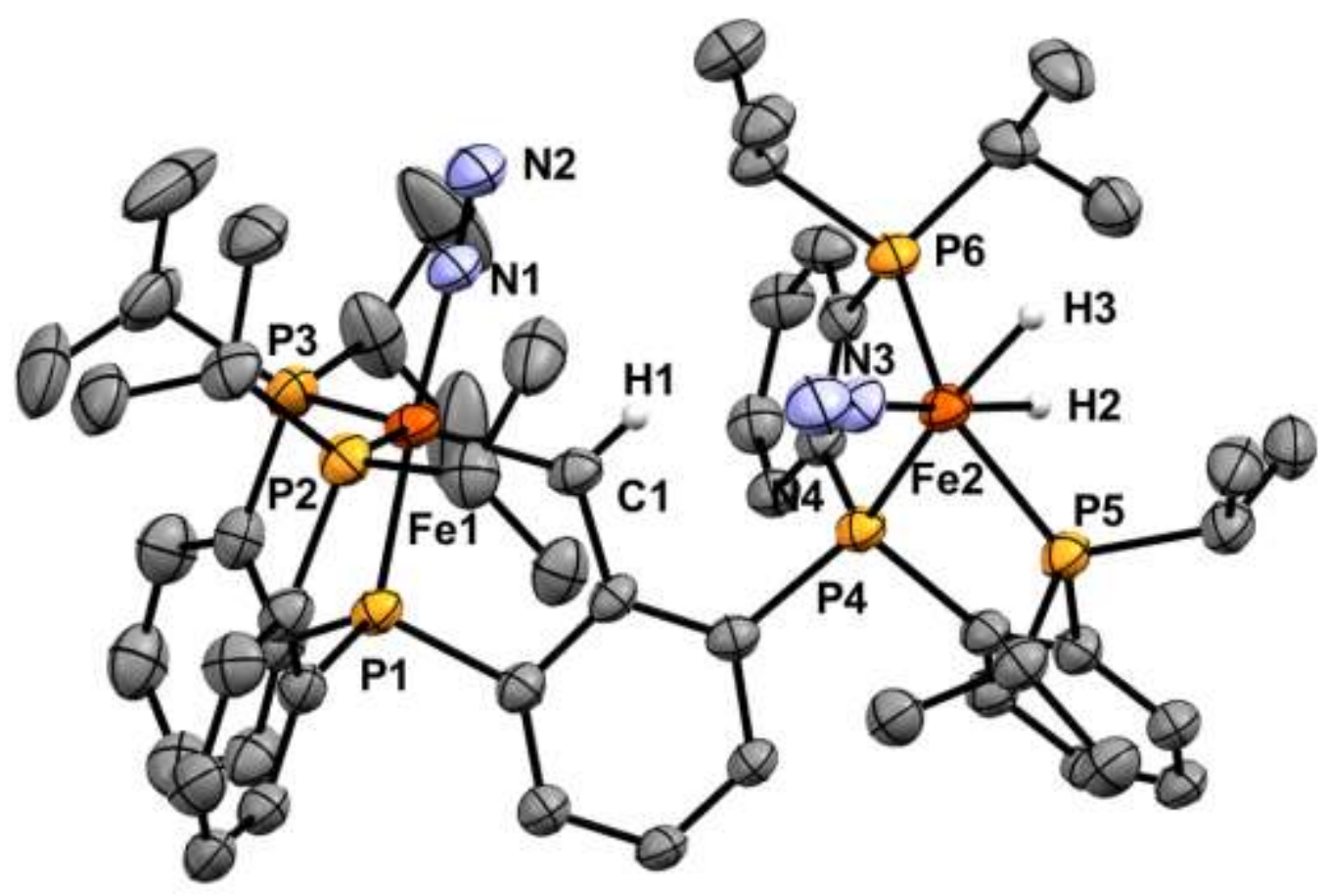

Figure S46. Crystal structure of $\left(\mathrm{P}_{6} \mathrm{ArCH}\right) \mathrm{Fe}_{2}\left(\mathrm{~N}_{2}\right)_{2}(\mathrm{H})_{2}(8)$. Ellipsoids are shown at the 50\% probability level. Hydrogen atoms and co-crystallized solvent molecules are not shown for clarity. 


\section{$\underline{\text { Reactivity with } \mathrm{H}_{2} \text { and } \mathrm{N}_{2}}$}

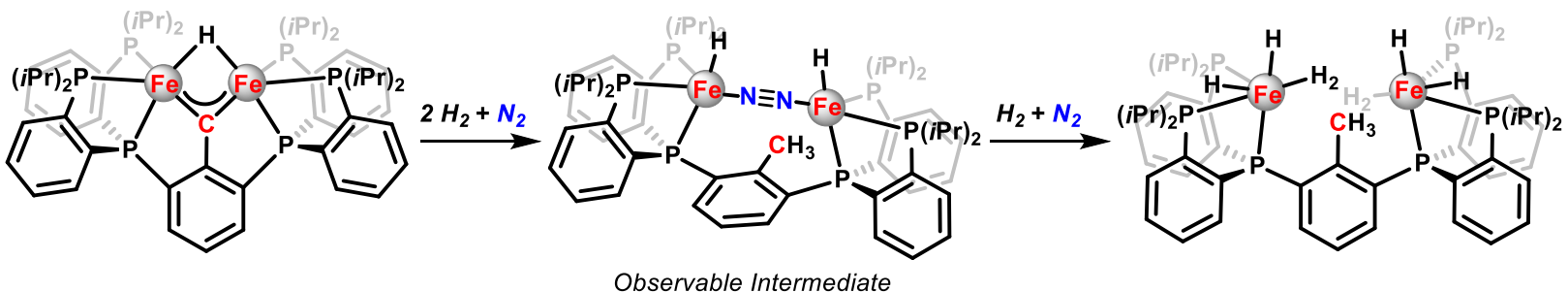

Reaction of 5 with $\mathbf{H}_{2} / \mathbf{N}_{2}$ in Sealed J Young tube. A J Young NMR tube was charged with 5 (12.5 mg, $0.012 \mathrm{mmol})$ and dissolved in $\mathrm{C}_{6} \mathrm{D}_{6}(0.45 \mathrm{~mL})$. The tube was degassed on the Schlenk line with three freeze-pump-thaw cycles. A mixture of $\mathrm{H}_{2} / \mathrm{N}_{2}$ (c.a.50:50) was admitted to the tube at room temperature. ${ }^{1} \mathrm{H}$ and ${ }^{31} \mathrm{P}$ NMR spectra were collected over the course of the reaction. Complete conversion of 5 to the final product $\left(\mathrm{P}_{6} \mathrm{ArCH}_{3}\right) \mathrm{Fe}_{2}\left(\mathrm{H}_{2}\right)_{2}(\mathrm{H})_{4}\left(\mathbf{6}-\mathbf{H}_{2}\right)$ is observed after 78 days. At early time points (75 min-24 hrs), $\left[\mathrm{P}_{6} \mathrm{ArCH}_{3}\right](\mathrm{FeH})_{2}\left(\mu-\mathrm{N}_{2}\right)(7)$ can be observed as an intermediate in the reaction.
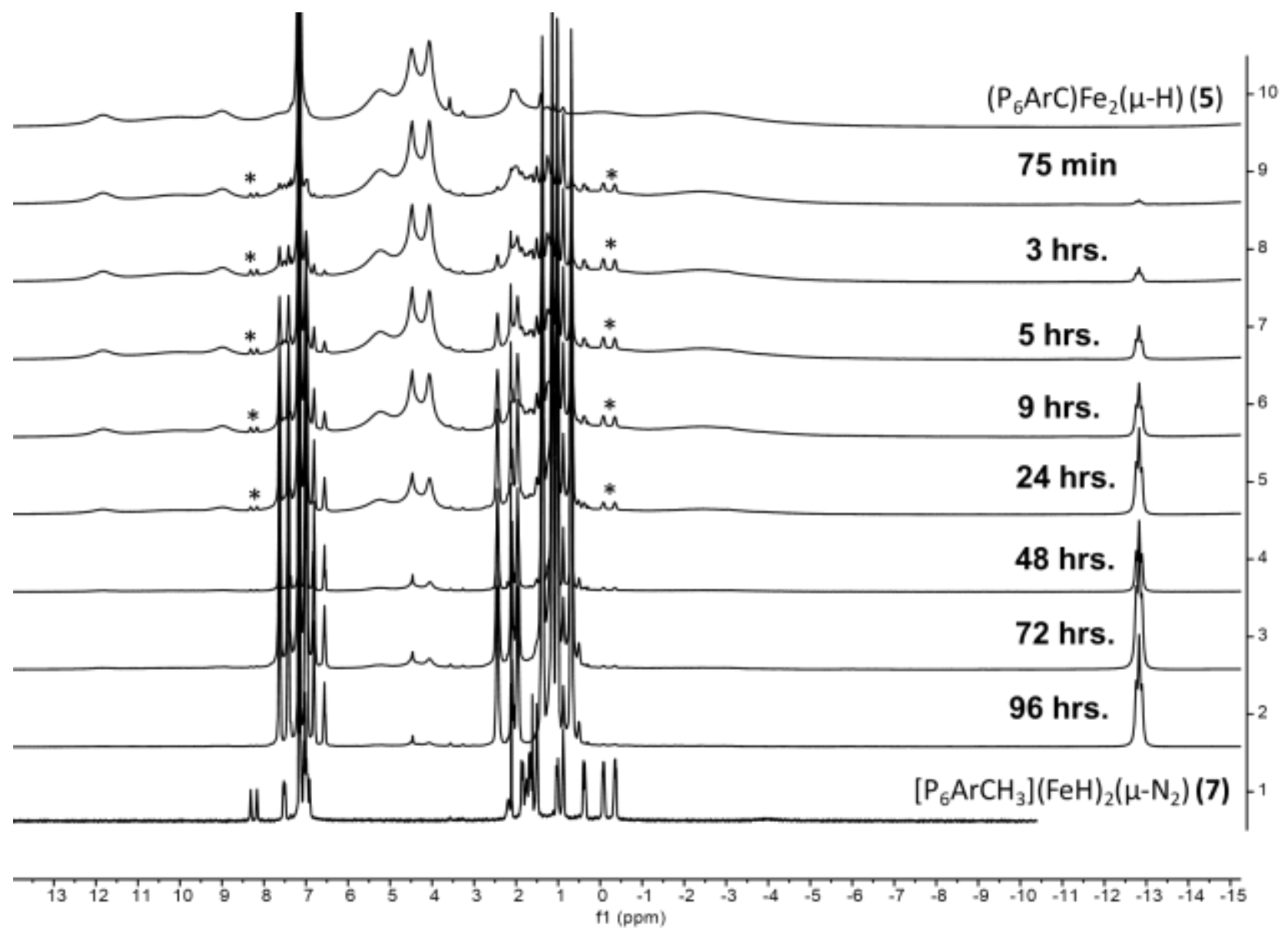

Figure S47. ${ }^{1} \mathrm{H}$ NMR spectra collected during the course of the reaction of $\left(\mathrm{P}_{6} \mathrm{ArC}\right) \mathrm{Fe}{ }_{2} \mathrm{H}(5)$ with a mixture of $\mathrm{H}_{2}$ and $\mathrm{N}_{2}$ in a sealed $\mathrm{J}$ Young tube. Asterisks denote characteristic resonances of $\left[\mathrm{P}_{6} \mathrm{ArCH}_{3}\right](\mathrm{FeH})_{2}\left(\mu-\mathrm{N}_{2}\right)(7)$, observed as an intermediate in the reaction. 

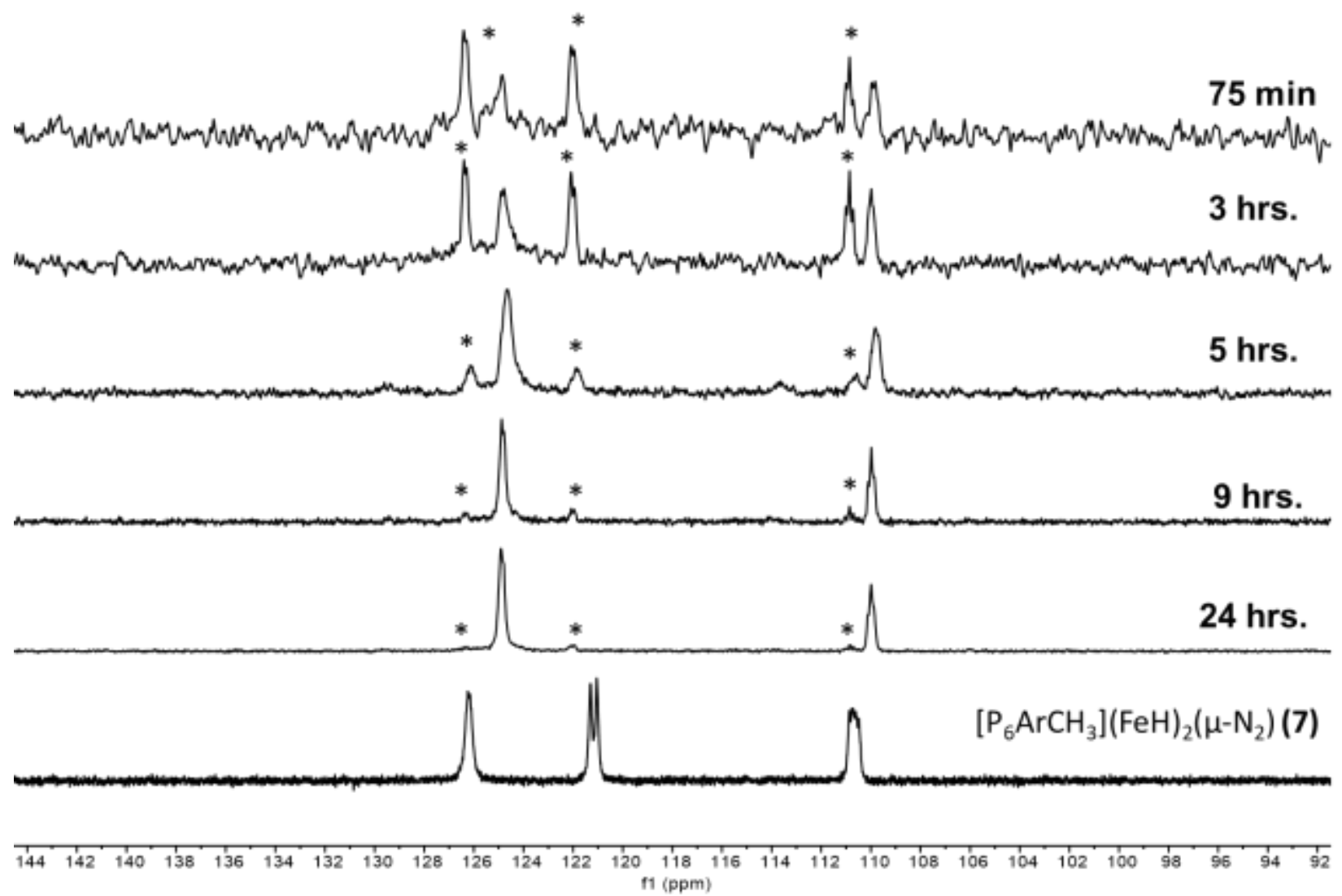

Figure S48. ${ }^{31} \mathrm{P}$ NMR spectra collected during the course of the reaction of $\left(\mathrm{P}_{6} \mathrm{ArC}\right) \mathrm{Fe} 2 \mathrm{H}(5)$ with a mixture of $\mathrm{H}_{2}$ and $\mathrm{N}_{2}$ in a sealed $\mathrm{J}$ Young tube. Asterisks denote characteristic resonances of $\left[\mathrm{P}_{6} \mathrm{ArCH}_{3}\right](\mathrm{FeH})_{2}\left(\mu-\mathrm{N}_{2}\right)(7)$, observed as an intermediate in the reaction. 


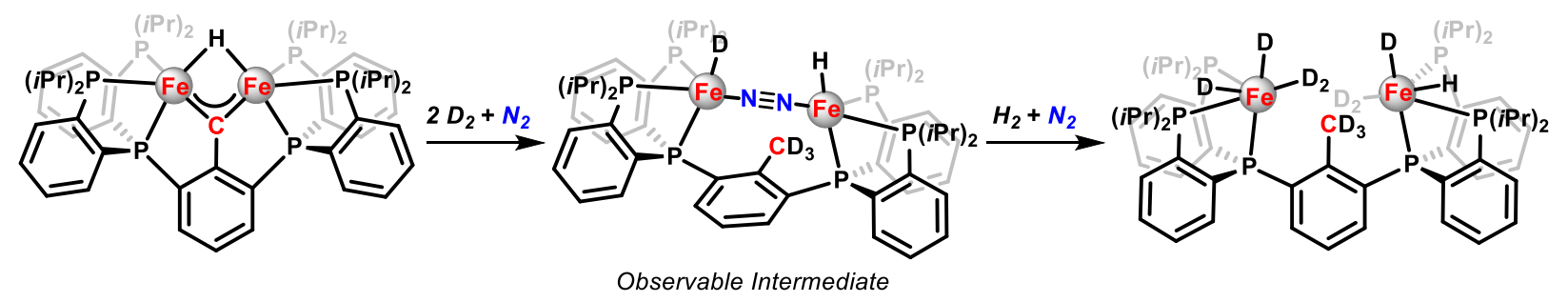

Reaction of 5 with $\mathbf{D}_{2} / \mathbf{N}_{2}$ in Sealed $\mathbf{J}$ Young tube. A J Young NMR tube was charged with $\mathbf{5}$ $(12.3 \mathrm{mg}, 0.012 \mathrm{mmol})$ and dissolved in $\mathrm{C}_{6} \mathrm{D}_{6}(0.45 \mathrm{~mL})$. The tube was degassed on the Schlenk line with three freeze-pump-thaw cycles. A mixture of $\mathrm{D}_{2} / \mathrm{N}_{2}$ (c.a.50:50) was admitted to the tube at room temperature. ${ }^{1} \mathrm{H}$ and ${ }^{31} \mathrm{P}$ NMR spectra were collected over the course of the reaction. Complete conversion of 5 to the final product $\left.\left(\mathrm{P}_{6} \mathrm{ArCH}_{3}\right) \mathrm{Fe}_{2}\left(\mathrm{D}_{2}\right)_{2}(\mathrm{D})_{3}(\mathrm{H}) \mathbf{( 6 - \mathbf { H }} / \mathbf{D}_{2}\right)$ is observed after 7-8 days. There is a significant isotope effect on the timecourse of the reaction. In the case of $\mathrm{H}_{2}$, the intermediate $\left[\mathrm{P}_{6} \mathrm{ArCH}_{3}\right](\mathrm{FeH})_{2}\left(\mu-\mathrm{N}_{2}\right)(7)$ is mostly consumed within 48 hours. However, for the reaction with $\mathrm{D}_{2},\left[\mathrm{P}_{6} \mathrm{ArCD}_{3}\right](\mathrm{FeX})_{2}\left(\mu-\mathrm{N}_{2}\right)(\mathbf{7}-\mathbf{H} / \mathbf{D}, \mathrm{X}=\mathrm{H}$ or $\mathrm{D})$ accumulates to a much great degree and is not fully consumed even after 120 hours. Notably, a hydridic/HD resonance is still observed at -12.9 which integrates to $1 \mathrm{H}$ in the final spectrum. Correspondingly, there is a lack of a $-\mathrm{CHD}_{2}$ resonance from the central methyl group. These observations indicate that the first equivalent of $\mathrm{D}_{2}$ adds regioselectively across the Fe-C bond such that the original hydride ligand remains bound to Fe and is not delivered to the carbyne carbon. Under thermal conditions, this reaction is not reversible and the hydride ligand does not exchange with $\mathrm{D}_{2}$ in the headspace. This rules out $\mathrm{C}-\mathrm{H}$ reductive elimination/oxidative addition of $\mathrm{H}_{2}$ as a mechanism for $\mathrm{H}_{2}$ activation by 5 . 

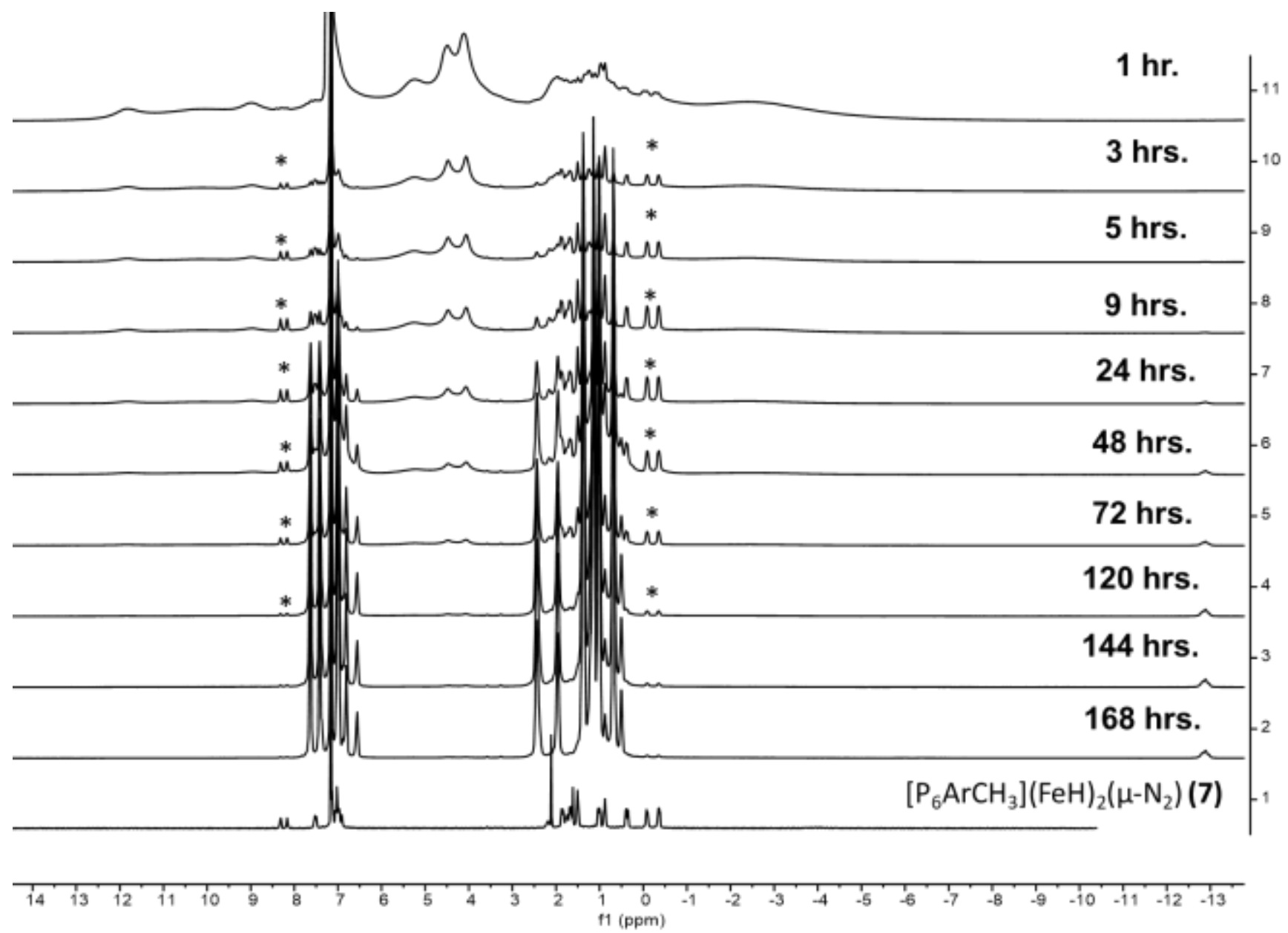

Figure S49. ${ }^{1} \mathrm{H}$ NMR spectra collected during the course of the reaction of $\left(\mathrm{P}_{6} \mathrm{ArC}\right) \mathrm{Fe} 2 \mathrm{H}(5)$ with a mixture of $\mathrm{D}_{2}$ and $\mathrm{N}_{2}$ in a sealed $\mathrm{J}$ Young tube. Asterisks denote characteristic resonances of $\left[\mathrm{P}_{6} \mathrm{ArCH}_{3}\right](\mathrm{FeX})_{2}\left(\mu-\mathrm{N}_{2}\right)(\mathbf{7 - H} / \mathbf{D}, \mathrm{X}=\mathrm{H}$ or D), observed as an intermediate in the reaction. 


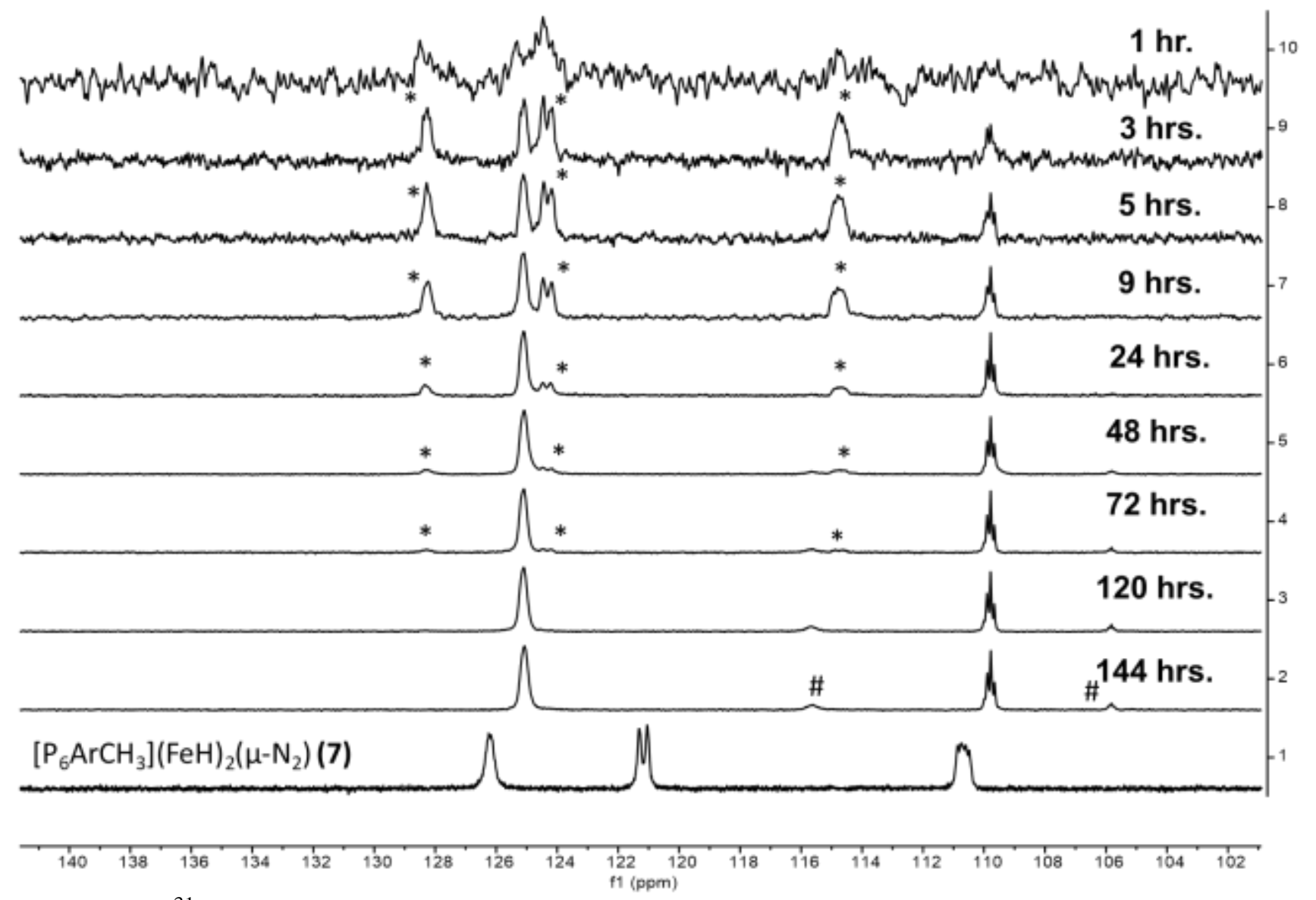

Figure S50. ${ }^{31} \mathrm{P}$ NMR spectra collected during the course of the reaction of $\left(\mathrm{P}_{6} \mathrm{ArC}\right) \mathrm{Fe}_{2} \mathrm{H}(5)$ with a mixture of $\mathrm{D}_{2}$ and $\mathrm{N}_{2}$ in a sealed $\mathrm{J}$ Young tube. Asterisks denote characteristic resonances of $\left[\mathrm{P}_{6} \mathrm{ArCH}_{3}\right](\mathrm{FeX})_{2}\left(\mu-\mathrm{N}_{2}\right)(\mathbf{7 - H} / \mathbf{D}, \mathrm{X}=\mathrm{H}$ or D), observed as an intermediate in the reaction. Note these resonances are shifted with respect to $\left[\mathrm{P}_{6} \mathrm{ArCH}_{3}\right](\mathrm{FeH})_{2}\left(\mu-\mathrm{N}_{2}\right)(7)$, which is not observed in the case of $\mathrm{H}_{2}$. The \# marked resonances correspond to $\left(\mathrm{P}_{6} \mathrm{ArCH}_{3}\right) \mathrm{Fe}_{2}\left(\mathrm{~N}_{2}\right)_{2}(\mathrm{D})_{3}(\mathrm{H})\left(\mathbf{6}-\mathrm{N}_{2}\right)$. 


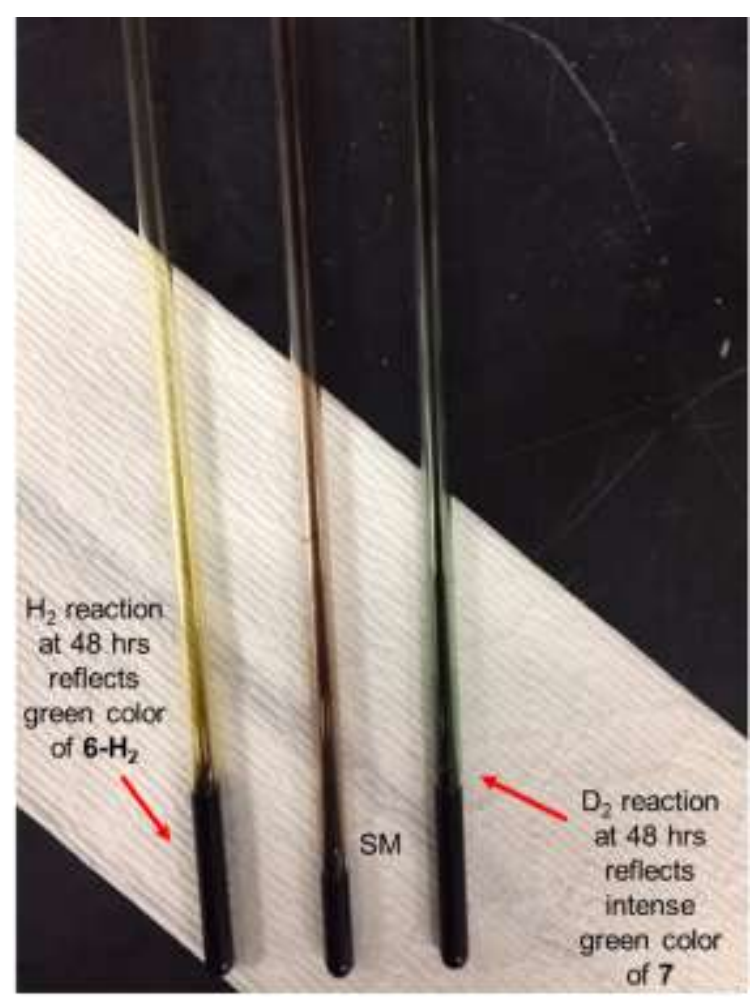

Figure S51. Comparison of colors of the reaction of $\mathrm{H}_{2}$ vs. $\mathrm{D}_{2}$ with $\left(\mathrm{P}_{6} \mathrm{ArC}\right) \mathrm{Fe}_{2} \mathrm{H}(\mathbf{5})$.

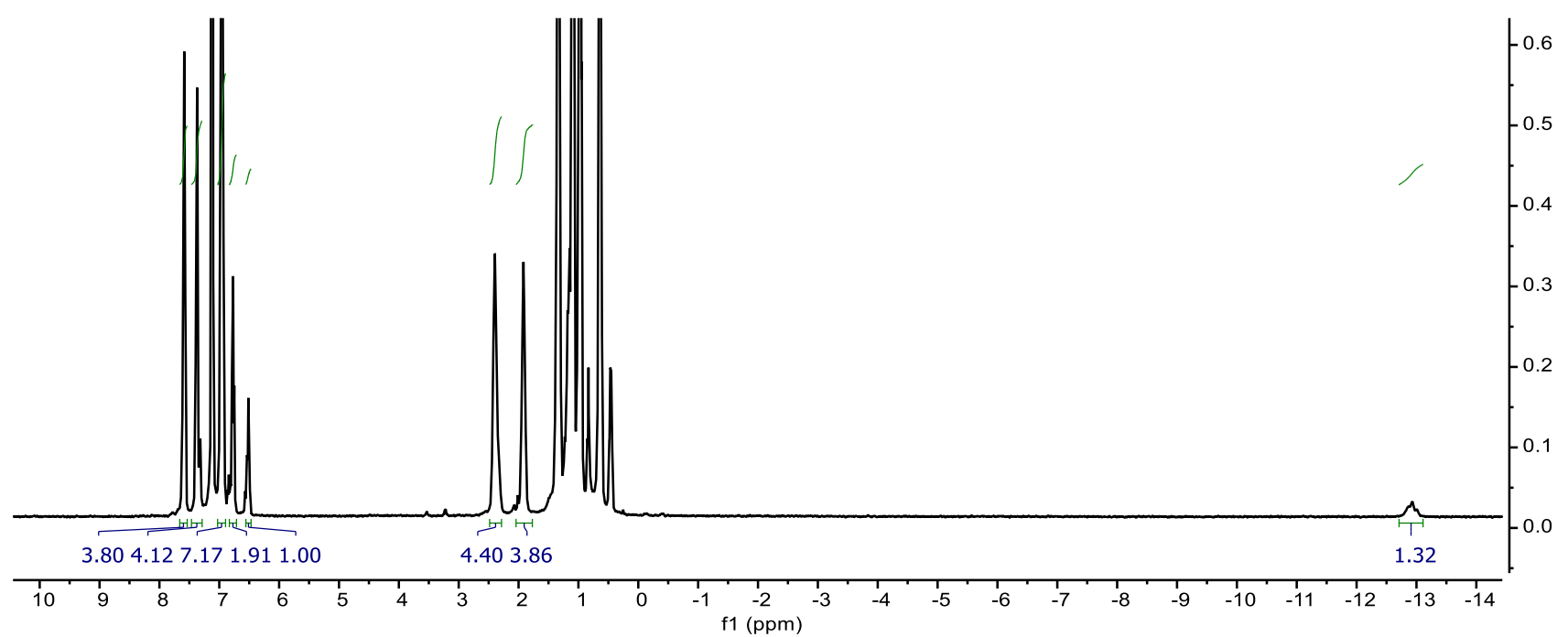

Figure S52. ${ }^{1} \mathrm{H}$ NMR spectra collected at the end of the reaction of $\left(\mathrm{P}_{6} \mathrm{ArC}\right) \mathrm{Fe}_{2} \mathrm{H}(5)$ with a mixture of $\mathrm{D}_{2}$ and $\mathrm{N}_{2}$ in a sealed $\mathrm{J}$ Young tube. Note especially the observation of the hydridic/HD resonance at -12.9 and the lack of a $-\mathrm{CHD}_{2}$ resonance from the central methyl group. Integration indicates that the first equivalent of $\mathrm{D}_{2}$ adds regioselectively across the $\mathrm{Fe}-\mathrm{C}$ bond such that the original hydride ligand remains bound to $\mathrm{Fe}$ and is not delivered to the carbyne carbon. Under thermal conditions, this reaction is not reversible and the hydride ligand does not exchange with $\mathrm{D}_{2}$ in the headspace. 


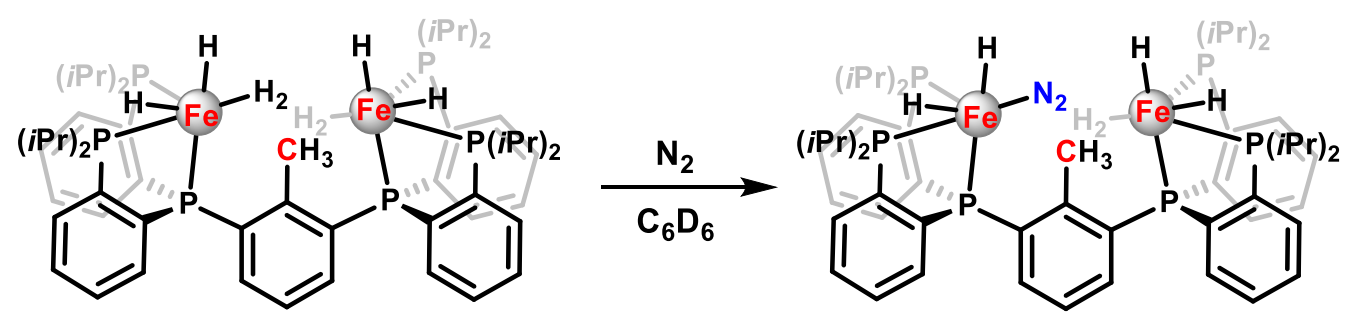

Conversion of 6- $\mathbf{H}_{2}$ to 6- $\mathbf{N}_{2}$. After conversion to $\left(\mathrm{P}_{6} \mathrm{ArCH}_{3}\right) \mathrm{Fe}_{2}\left(\mathrm{H}_{2}\right)_{2}(\mathrm{H})_{4}\left(\mathbf{6}-\mathbf{H}_{2}\right)$ is complete, a $\mathbf{J}$ Young tube containing $\left(\mathrm{P}_{6} \mathrm{ArCH}_{3}\right) \mathrm{Fe}_{2}\left(\mathrm{H}_{2}\right)_{2}(\mathrm{H})_{4}\left(\mathbf{6}-\mathrm{H}_{2}\right)$ was degassed on the Schlenk line with three freeze-pump-thaw cycles and backfilled with $\mathrm{N}_{2}$. Over time, $\left(\mathrm{P}_{6} \mathrm{ArCH}_{3}\right) \mathrm{Fe}_{2}\left(\mathrm{H}_{2}\right)_{2}(\mathrm{H})_{4}\left(\mathbf{6}-\mathbf{H}_{2}\right)$ then converts to $\left(\mathrm{P}_{6} \mathrm{ArCH}_{3}\right) \mathrm{Fe}_{2}\left(\mathrm{~N}_{2}\right)_{2}(\mathrm{H})_{4}\left(\mathbf{6}-\mathrm{N}_{2}\right)$, which can be followed by ${ }^{1} \mathrm{H}$ and ${ }^{31} \mathrm{P}$ NMR.

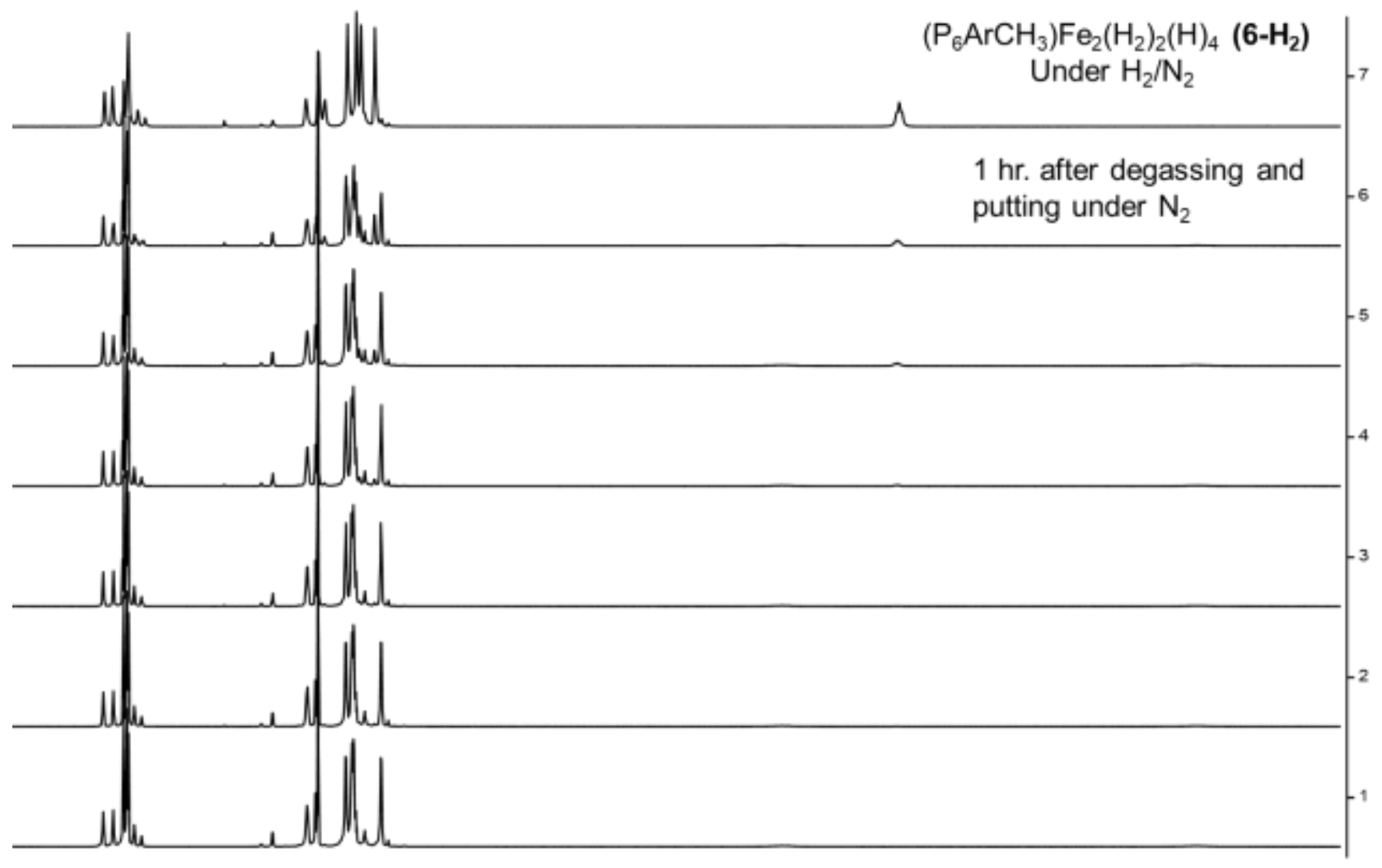

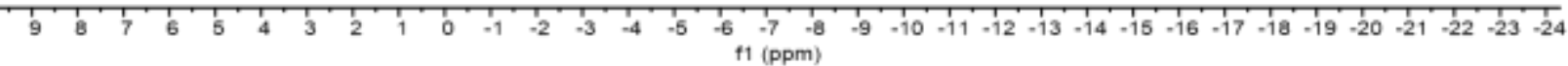

Figure S53. ${ }^{1} \mathrm{H}$ NMR spectra collected during the course of converting of $\left(\mathrm{P}_{6} \mathrm{ArCH}_{3}\right) \mathrm{Fe}_{2}\left(\mathrm{H}_{2}\right)_{2}(\mathrm{H})_{4}$ (6- $\left.\mathbf{H}_{2}\right)$ to $\left(\mathrm{P}_{6} \mathrm{ArCH}_{3}\right) \mathrm{Fe}_{2}\left(\mathrm{~N}_{2}\right)_{2}(\mathrm{H})_{4}\left(\mathbf{6}-\mathrm{N}_{2}\right)$ under $\mathrm{N}_{2}$ in a sealed $\mathbf{J}$ Young tube. 


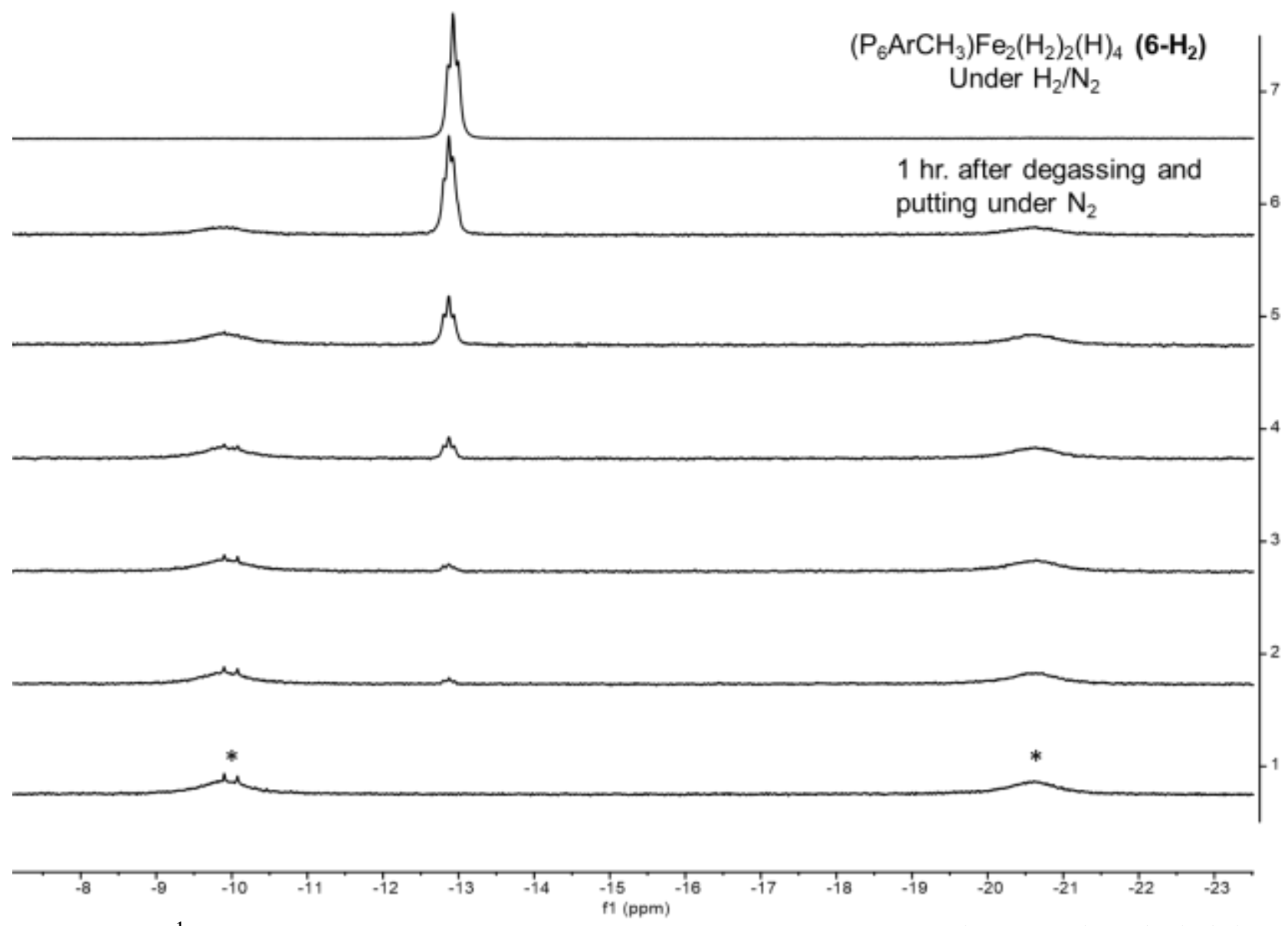

Figure S54. ${ }^{1} \mathrm{H}$ NMR spectra collected during the course of converting of $\left(\mathrm{P}_{6} \mathrm{ArCH}_{3}\right) \mathrm{Fe}_{2}\left(\mathrm{H}_{2}\right)_{2}(\mathrm{H})_{4}$ (6-- 2$)$ to $\left(\mathrm{P}_{6} \mathrm{ArCH}_{3}\right) \mathrm{Fe}_{2}\left(\mathrm{~N}_{2}\right)_{2}(\mathrm{H})_{4}\left(\mathbf{6}-\mathrm{N}_{2}\right)$ under $\mathrm{N}_{2}$ in a sealed $\mathrm{J}$ Young tube, focusing on the hydridic region. Asterisks denote the position of the resonances for $\mathbf{6}-\mathbf{N}_{2}$. 

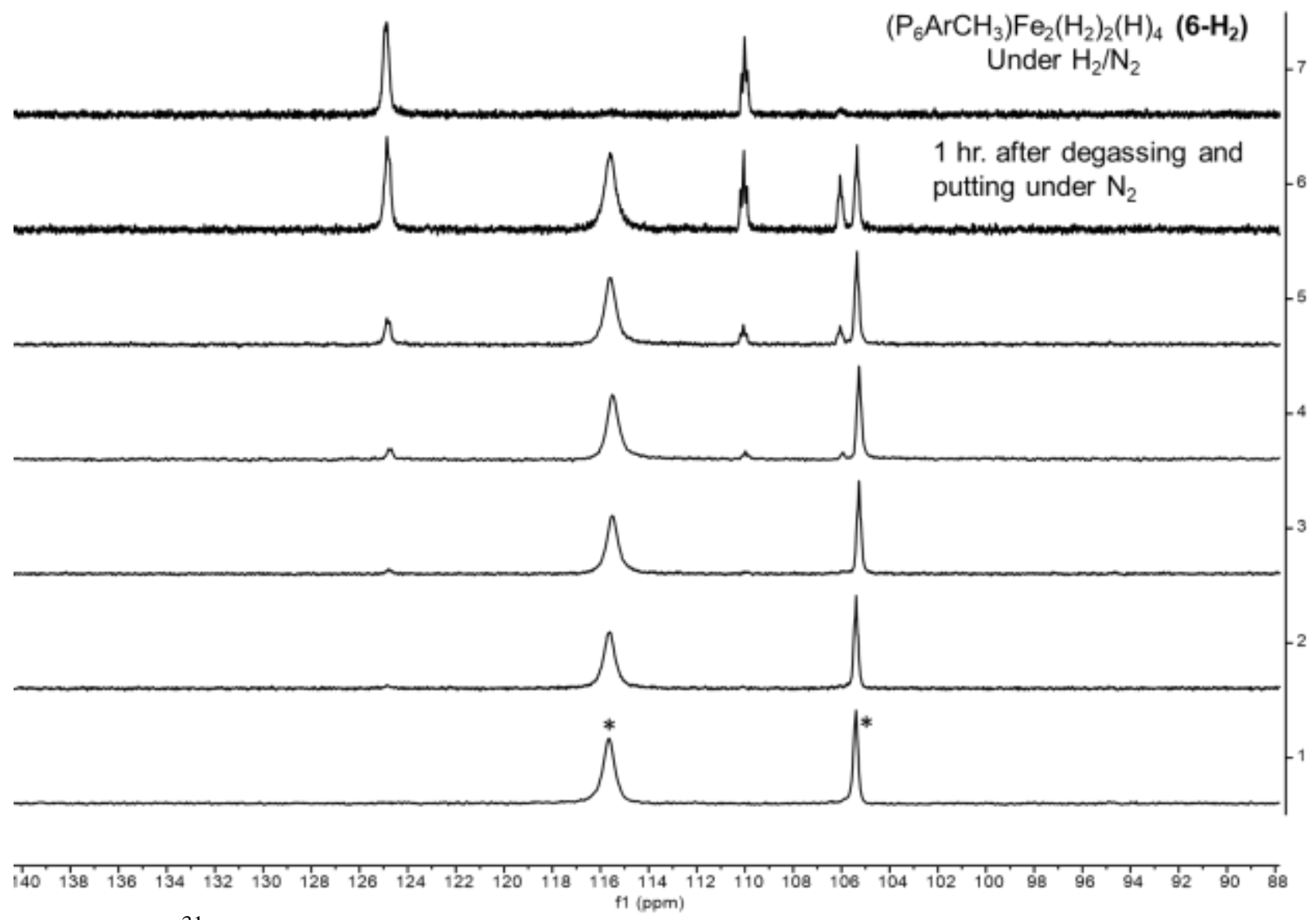

Figure S55. ${ }^{31} \mathrm{P}$ NMR spectra collected during the course of converting of $\left(\mathrm{P}_{6} \mathrm{ArCH}_{3}\right) \mathrm{Fe}_{2}\left(\mathrm{H}_{2}\right)_{2}(\mathrm{H})_{4}$ (6-- 2$)$ to $\left(\mathrm{P}_{6} \mathrm{ArCH}_{3}\right) \mathrm{Fe}_{2}\left(\mathrm{~N}_{2}\right)_{2}(\mathrm{H})_{4}\left(6-\mathrm{N}_{2}\right)$ under $\mathrm{N}_{2}$ in a sealed $\mathrm{J}$ Young tube. Asterisks denote the position of the resonances for $\mathbf{6}-\mathbf{N}_{2}$. 


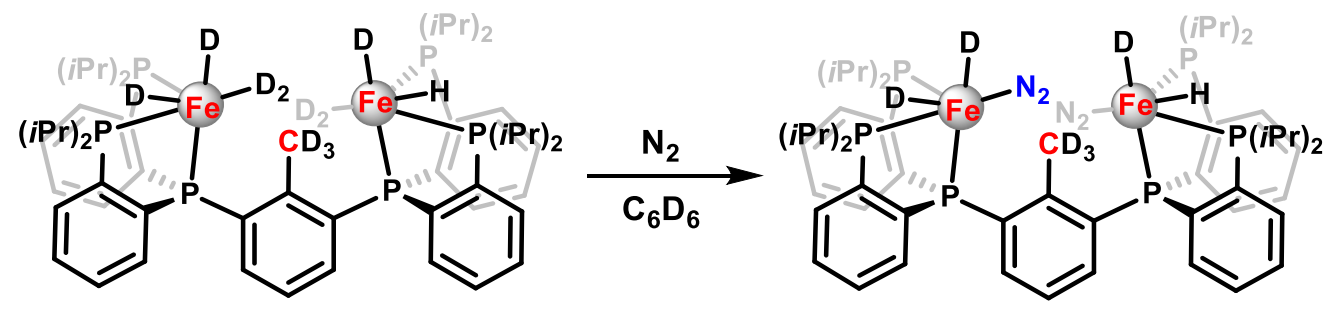

Conversion of 6- $\mathbf{D}_{2}$ to 6- $\mathbf{N}_{2}$. After conversion to $\left(\mathrm{P}_{6} \mathrm{ArCD}_{3}\right) \mathrm{Fe}_{2}\left(\mathrm{D}_{2}\right)_{2}(\mathrm{D})_{3}(\mathrm{H})\left(\mathbf{6}-\mathrm{D}_{2}\right)$ is complete, a J Young tube containing $\left(\mathrm{P}_{6} \mathrm{ArCD}_{3}\right) \mathrm{Fe}_{2}\left(\mathrm{D}_{2}\right)_{2}(\mathrm{D})_{3}(\mathrm{H})\left(\mathbf{6}-\mathrm{D}_{2}\right)$ was degassed on the Schlenk line with three freeze-pump-thaw cycles and backfilled with $\mathrm{N}_{2}$. Over time, $\left(\mathrm{P}_{6} \mathrm{ArCD}_{3}\right) \mathrm{Fe}_{2}\left(\mathrm{D}_{2}\right)_{2}(\mathrm{D})_{3}(\mathrm{H})$ $\left(\mathbf{6}-\mathrm{D}_{2}\right)$ then converts to $\left(\mathrm{P}_{6} \mathrm{ArCD}_{3}\right) \mathrm{Fe}_{2}\left(\mathrm{~N}_{2}\right)_{2}(\mathrm{D})_{3}(\mathrm{H})\left(\mathbf{6}-\mathrm{N}_{2}\right)$, which can be followed by ${ }^{1} \mathrm{H}$ and ${ }^{31} \mathrm{P}$ NMR.
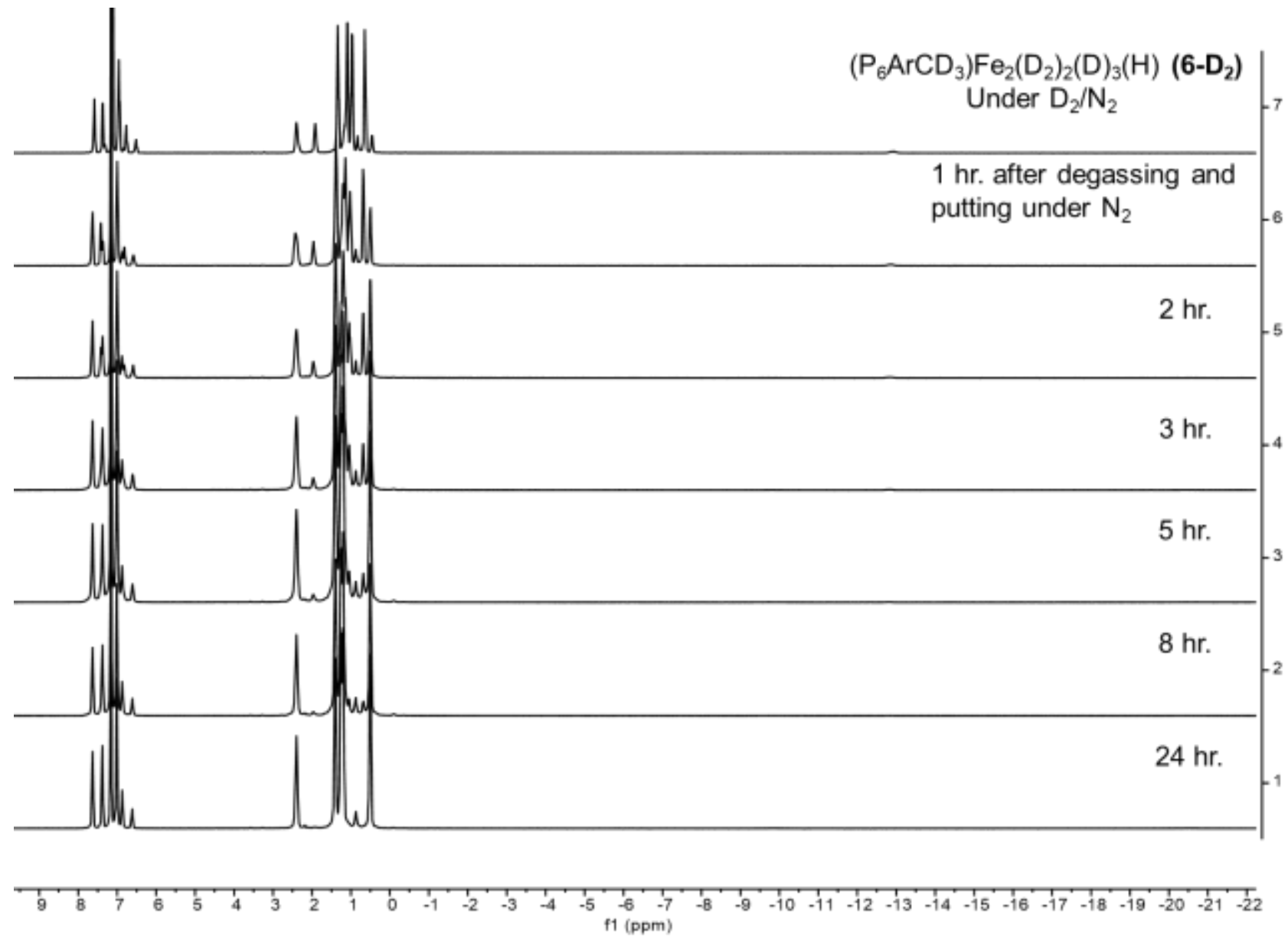

Figure S56. ${ }^{1} \mathrm{H}$ NMR spectra collected during the course of converting of $\left(\mathrm{P}_{6} \mathrm{ArCD}_{3}\right) \mathrm{Fe}_{2}\left(\mathrm{D}_{2}\right)_{2}(\mathrm{D})_{3}(\mathrm{H})\left(\mathbf{6}-\mathrm{D}_{2}\right)$ to $\left(\mathrm{P}_{6} \mathrm{ArCD}_{3}\right) \mathrm{Fe}_{2}\left(\mathrm{~N}_{2}\right)_{2}(\mathrm{D})_{3}(\mathrm{H})\left(\mathbf{6}-\mathbf{N}_{2}\right)$, under $\mathrm{N}_{2}$ in a sealed $\mathbf{J}$ Young tube. 


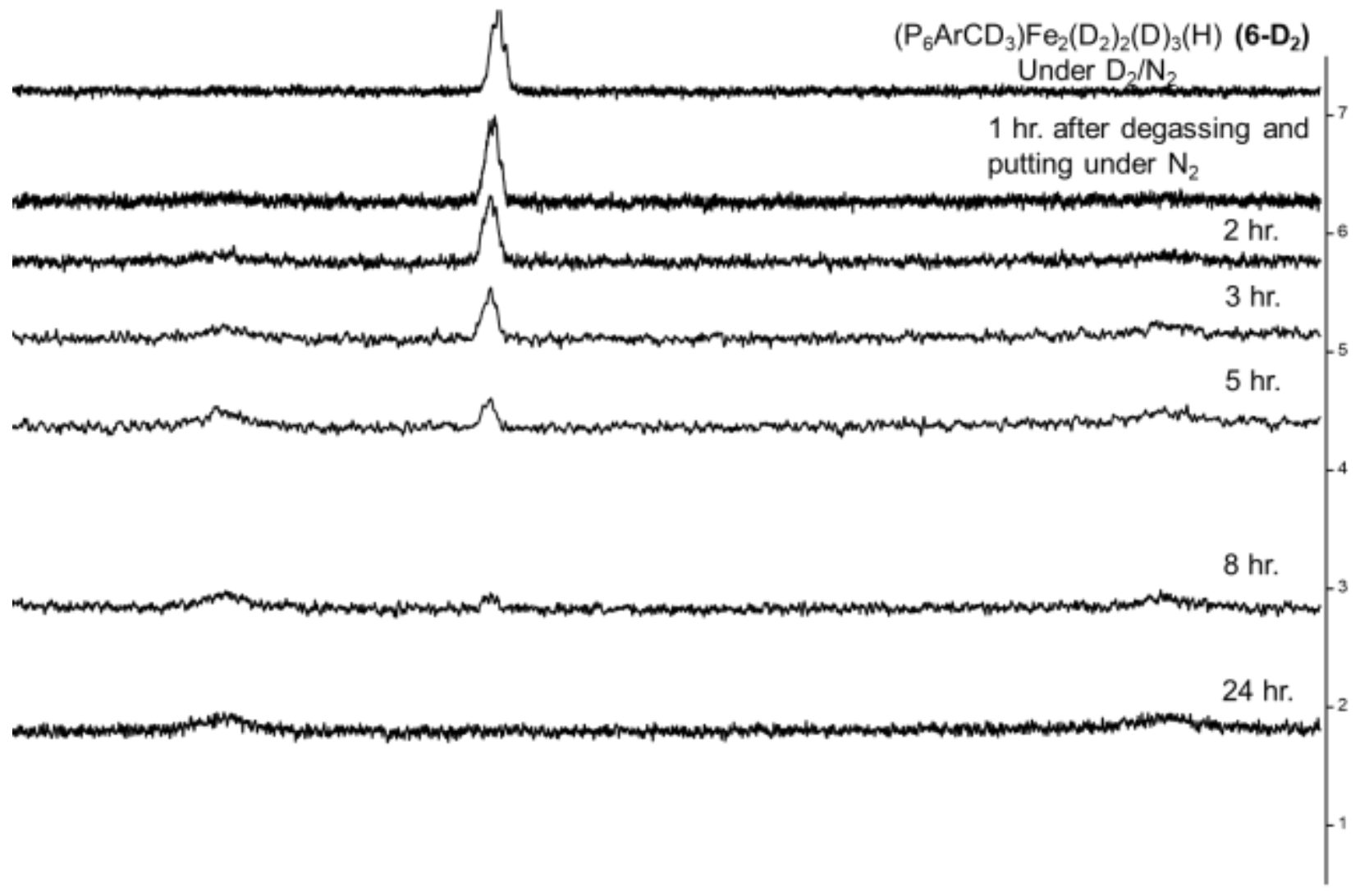

\begin{tabular}{|c|c|c|c|c|c|c|c|c|c|c|c|c|c|c|}
\hline-8 & $\begin{array}{l}1 \\
-9\end{array}$ & $=10$ & $=11$ & -12 & -13 & -14 & $\begin{array}{c}1 \\
=15 \\
f 1(\mathrm{ppm})\end{array}$ & -16 & -17 & -18 & -19 & $\frac{1}{-20}$ & -21 & -22 \\
\hline
\end{tabular}

Figure S57. ${ }^{1} \mathrm{H}$ NMR spectra collected during the course of converting of $\left(\mathrm{P}_{6} \mathrm{ArCD}_{3}\right) \mathrm{Fe}_{2}\left(\mathrm{D}_{2}\right)_{2}(\mathrm{D})_{3}(\mathrm{H})\left(\mathbf{6}-\mathrm{D}_{2}\right)$ to $\left(\mathrm{P}_{6} \mathrm{ArCD}_{3}\right) \mathrm{Fe}_{2}\left(\mathrm{~N}_{2}\right)_{2}(\mathrm{D})_{3}(\mathrm{H})\left(\mathbf{6}-\mathbf{N}_{2}\right)$, under $\mathrm{N}_{2}$ in a sealed $\mathbf{J}$ Young tube, focusing on the hydridic region. 

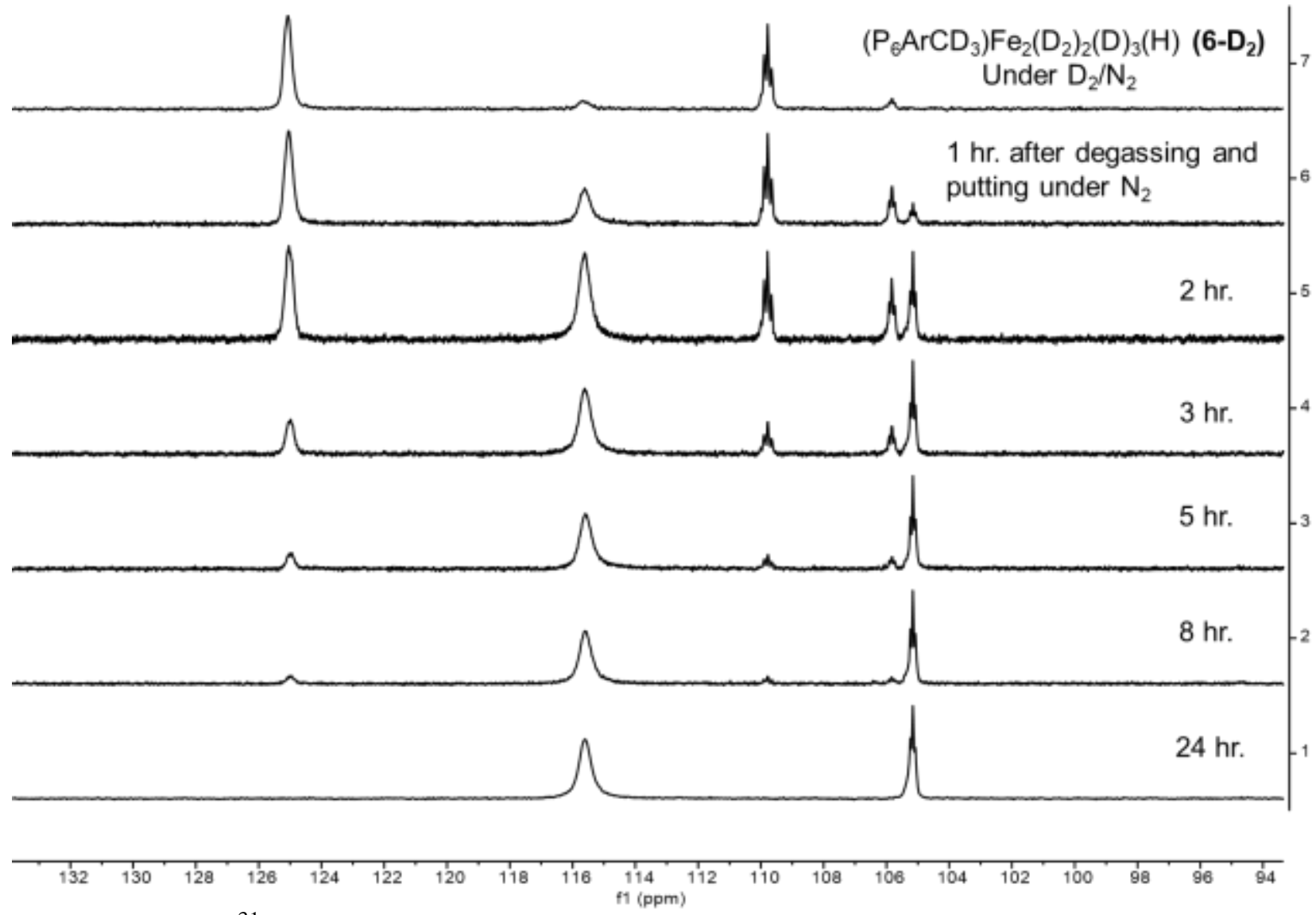

Figure S58. ${ }^{31} \mathrm{P}$ NMR spectra collected during the course of converting of $\left(\mathrm{P}_{6} \mathrm{ArCD}_{3}\right) \mathrm{Fe}_{2}\left(\mathrm{D}_{2}\right)_{2}(\mathrm{D})_{3}(\mathrm{H})\left(\mathbf{6}-\mathrm{D}_{2}\right)$ to $\left(\mathrm{P}_{6} \mathrm{ArCD}_{3}\right) \mathrm{Fe}_{2}\left(\mathrm{~N}_{2}\right)_{2}(\mathrm{D})_{3}(\mathrm{H})\left(\mathbf{6}-\mathrm{N}_{2}\right)$, under $\mathrm{N}_{2}$ in a sealed $\mathbf{J}$ Young tube. 

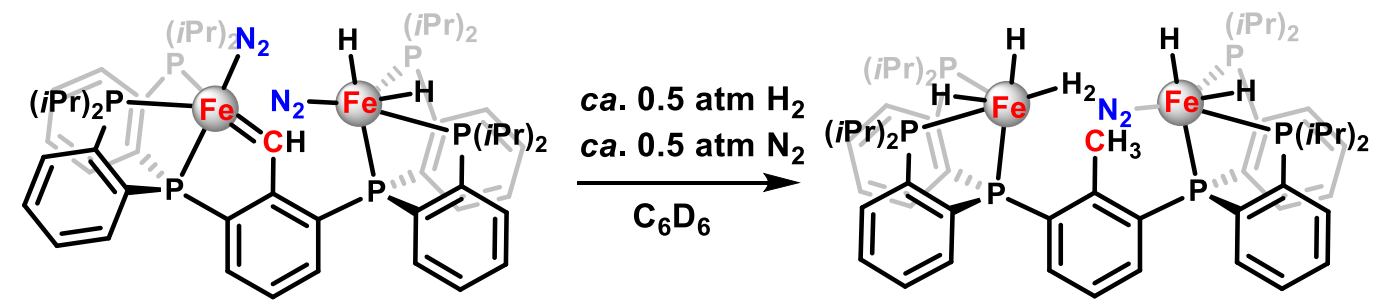

Reaction of 8 with $\mathbf{H}_{2} / \mathbf{N}_{2}$ in Sealed $\mathbf{J}$ Young tube. A J Young NMR tube was charged with 8 $(21.8 \mathrm{mg}, 0.020 \mathrm{mmol})$ and dissolved in $\mathrm{C}_{6} \mathrm{D}_{6}(0.45 \mathrm{~mL})$. The tube was degassed on the Schlenk line with three freeze-pump-thaw cycles. A mixture of $\mathrm{H}_{2} / \mathrm{N}_{2}$ (c.a.50:50) was admitted to the tube at room temperature. ${ }^{1} \mathrm{H}$ and ${ }^{31} \mathrm{P}$ NMR spectra were collected over the course of the reaction. Complete conversion of 8 to the final product $\left(\mathrm{P}_{6} \mathrm{ArCH}_{3}\right) \mathrm{Fe}_{2}\left(\mathrm{H}_{2}\right)\left(\mathrm{N}_{2}\right)(\mathrm{H})_{4}\left(\mathbf{6}-\mathbf{H}_{2} / \mathbf{N}_{2}\right)$ is observed after 1-2 hours based on loss of the diagnostic carbene $\mathrm{C}-\mathrm{H}$ resonance at $\sim 11 \mathrm{ppm}$ and changes in the ${ }^{31} \mathrm{P}$ NMR spectrum. The timescale of this reaction is significantly faster than that for $\mathbf{5}$ to be hydrogenated. Notably, the hydride resonances of $\mathbf{8}$ do not disappear in the course of the reaction and the ${ }^{31} \mathrm{P}$ NMR reflects 4-5 unique $\mathrm{P}$ environments. Together, this data indicates that $\mathrm{N}_{2}$ does not exchange with $\mathrm{H}_{2}$ under the experimental conditions.

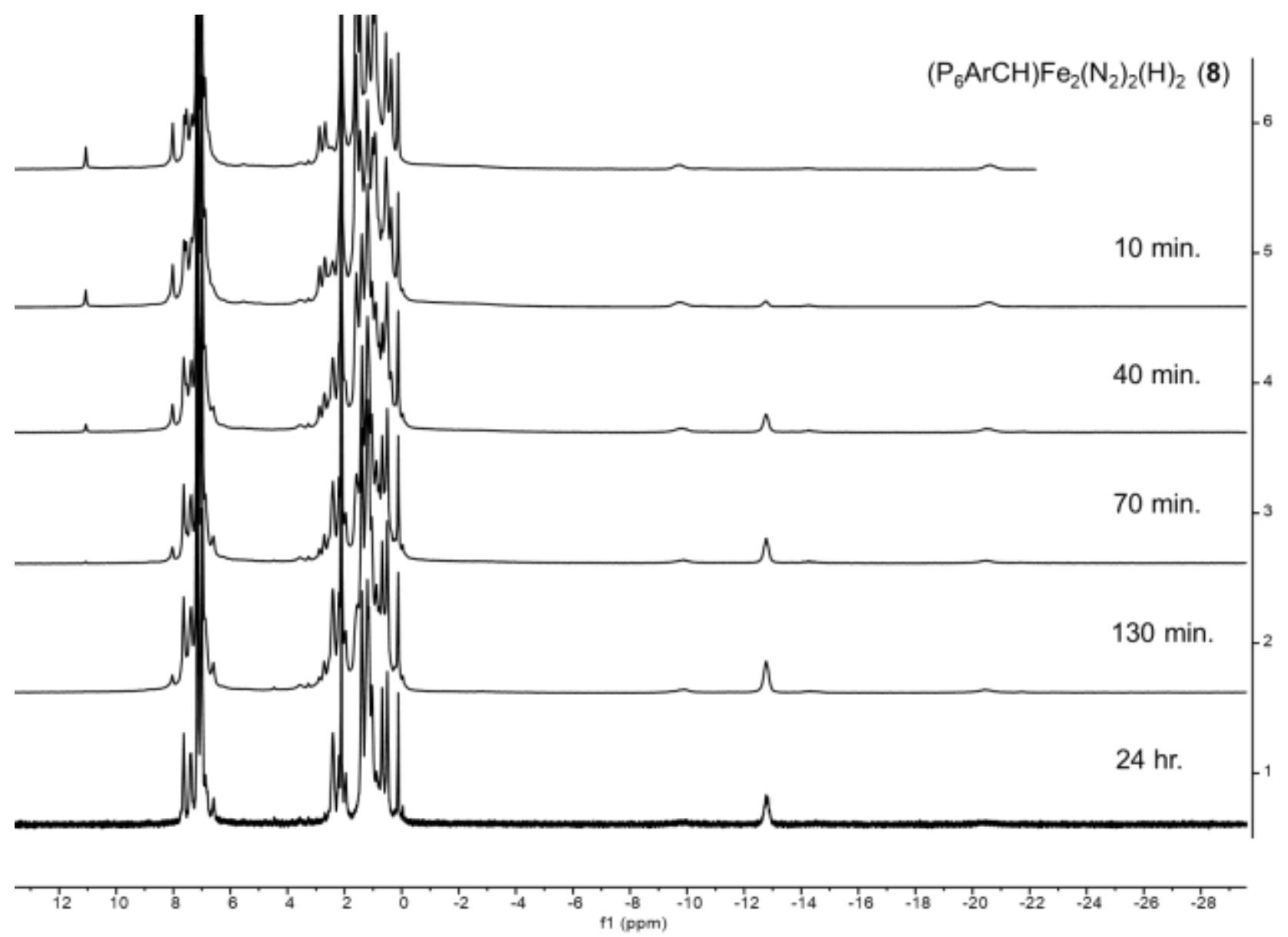

Figure S59. ${ }^{1} \mathrm{H}$ NMR spectra collected during the course of the reaction of $\left(\mathrm{P}_{6} \mathrm{ArCH}\right) \mathrm{Fe}_{2}\left(\mathrm{~N}_{2}\right)_{2}(\mathrm{H})_{2}$ (8) with a mixture of $\mathrm{H}_{2}$ and $\mathrm{N}_{2}$ in a sealed $\mathrm{J}$ Young tube. 


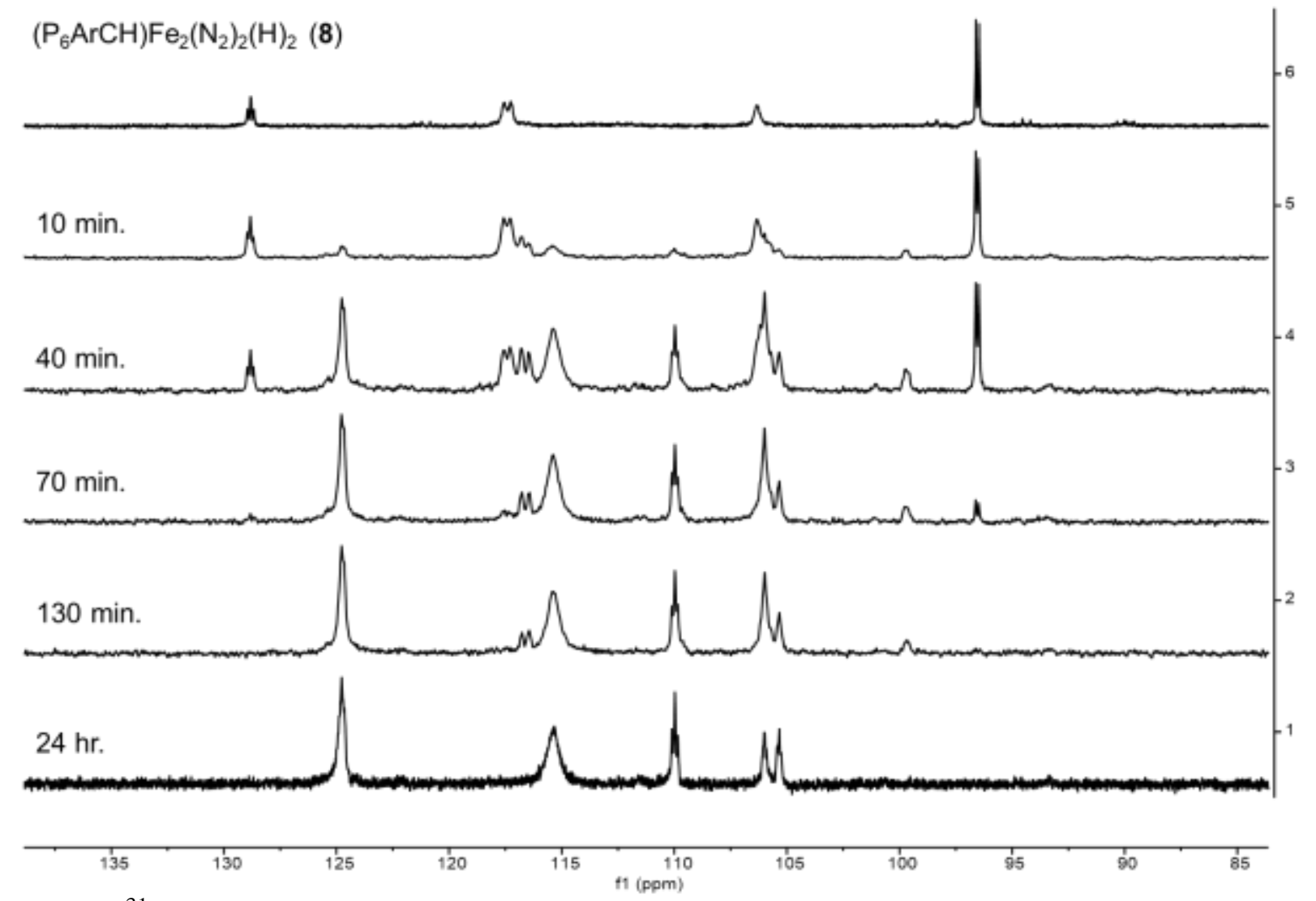

Figure S60. ${ }^{31} \mathrm{P}$ NMR spectra collected during the course of the reaction of $\left(\mathrm{P}_{6} \mathrm{ArCH}\right) \mathrm{Fe}_{2}\left(\mathrm{~N}_{2}\right)_{2}(\mathrm{H})_{2}$ (8) with a mixture of $\mathrm{H}_{2}$ and $\mathrm{N}_{2}$ in a sealed $\mathrm{J}$ Young tube. 


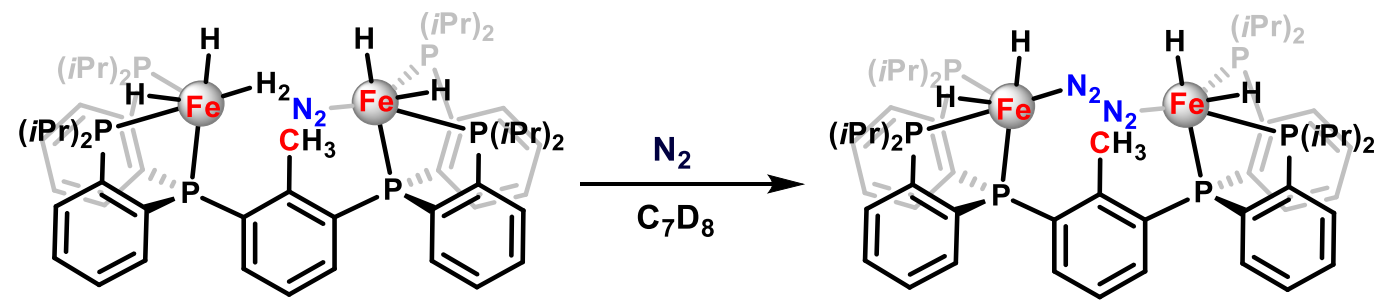

Conversion of 6- $\mathrm{H}_{2} / \mathrm{N}_{2}$ from 8 to 6-N2. After conversion of 8 to $\left(\mathrm{P}_{6} \mathrm{ArCH}_{3}\right) \mathrm{Fe}_{2}\left(\mathrm{H}_{2}\right)\left(\mathrm{N}_{2}\right)(\mathrm{H})_{4}$ (6$\left.\mathbf{H}_{2} / \mathbf{N}_{2}\right)$ is complete, a J Young tube containing $\left(\mathrm{P}_{6} \mathrm{ArCH}_{3}\right) \mathrm{Fe}_{2}\left(\mathrm{H}_{2}\right)\left(\mathrm{N}_{2}\right)(\mathrm{H})_{4}\left(\mathbf{6}-\mathbf{H}_{2} / \mathbf{N}_{2}\right)$ was degassed on the Schlenk line with three freeze-pump-thaw cycles and backfilled with $\mathrm{N}_{2}$. Over time, $\left(\mathrm{P}_{6} \mathrm{ArCH}_{3}\right) \mathrm{Fe}_{2}\left(\mathrm{H}_{2}\right)\left(\mathrm{N}_{2}\right)(\mathrm{H})_{4}\left(\mathbf{6}-\mathrm{H}_{2} / \mathbf{N}_{2}\right)$ then converts to $\left(\mathrm{P}_{6} \mathrm{ArCH}_{3}\right) \mathrm{Fe}_{2}\left(\mathrm{~N}_{2}\right)_{2}(\mathrm{H})_{4}\left(\mathbf{6}-\mathrm{N}_{2}\right)$, which can be followed by ${ }^{1} \mathrm{H}$ and ${ }^{31} \mathrm{P} \mathrm{NMR}$.
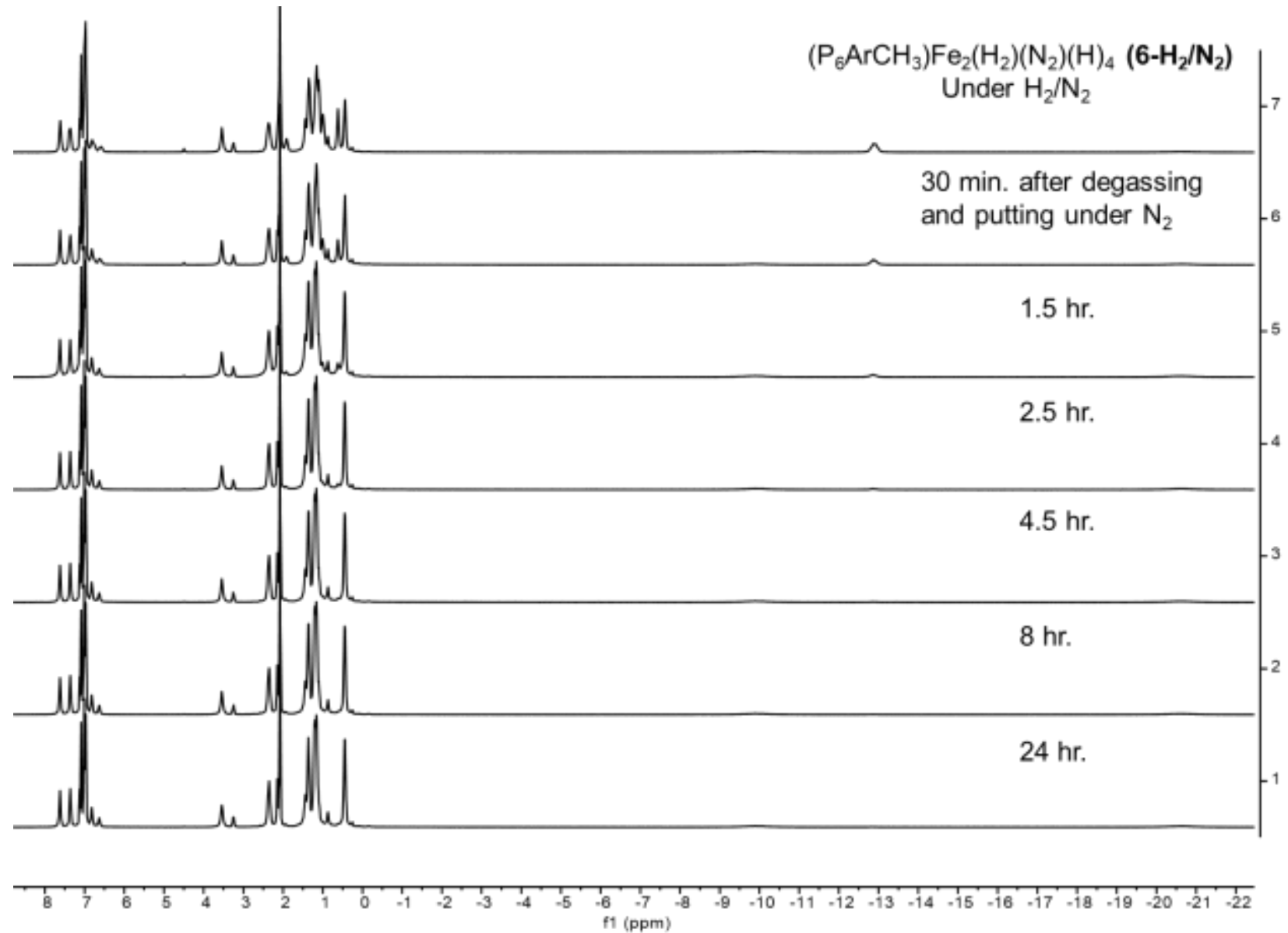

Figure S61. ${ }^{1} \mathrm{H}$ NMR spectra collected during the course of converting of $\left(\mathrm{P}_{6} \mathrm{ArCH}_{3}\right) \mathrm{Fe}_{2}\left(\mathrm{H}_{2}\right)\left(\mathrm{N}_{2}\right)(\mathrm{H})_{4}\left(\mathbf{6}-\mathbf{H}_{2} / \mathbf{N}_{2}\right)$ to $\left(\mathrm{P}_{6} \mathrm{ArCH}_{3}\right) \mathrm{Fe}_{2}\left(\mathrm{~N}_{2}\right)_{2}(\mathrm{H})_{4}\left(\mathbf{6}-\mathbf{N}_{2}\right)$, under $\mathrm{N}_{2}$ in a sealed $\mathbf{J}$ Young tube. 


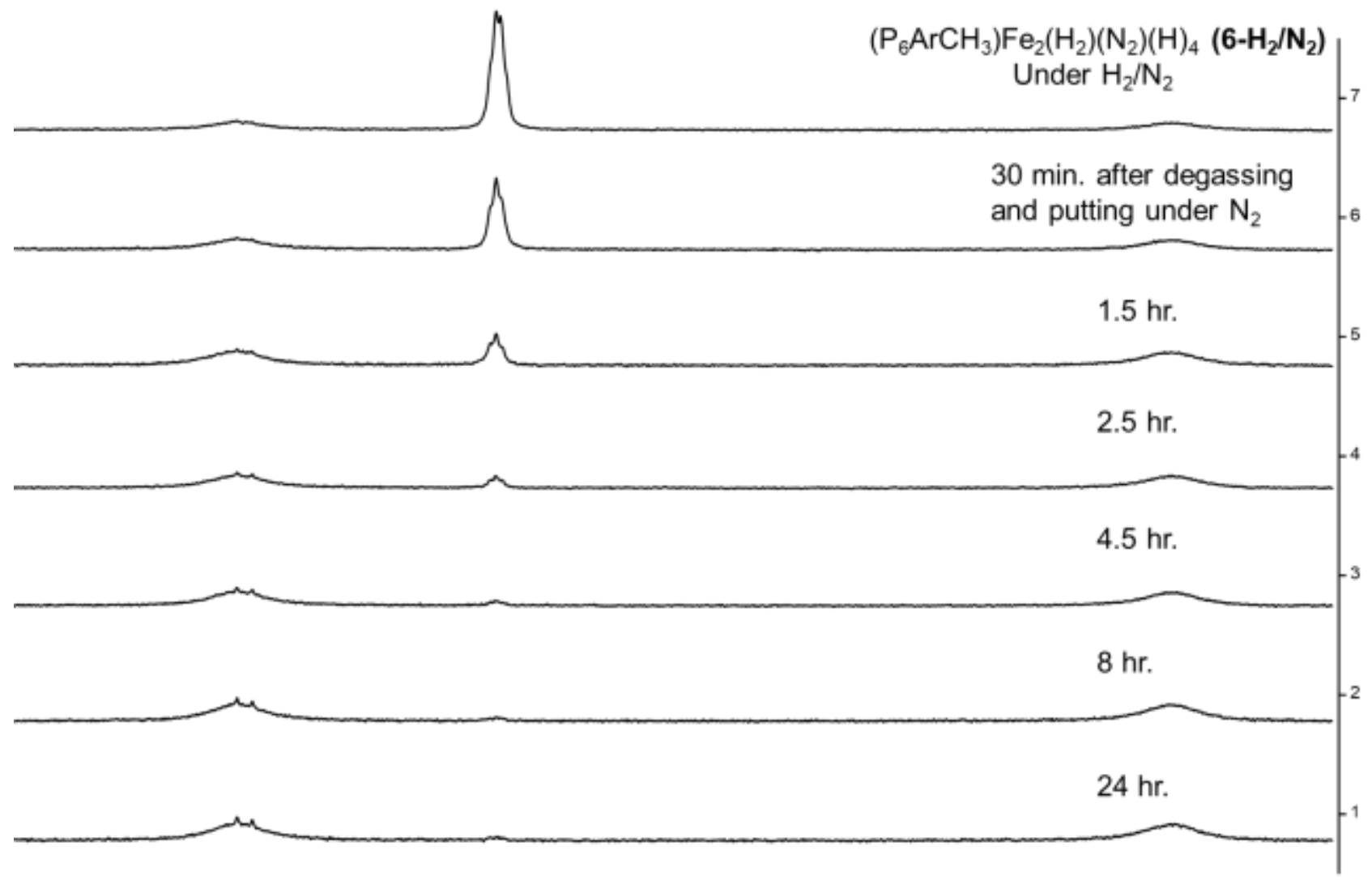

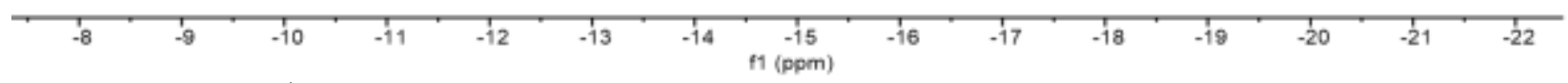

Figure S62. ${ }^{1} \mathrm{H}$ NMR spectra collected during the course of converting of $\left(\mathrm{P}_{6} \mathrm{ArCH}_{3}\right) \mathrm{Fe}_{2}\left(\mathrm{H}_{2}\right)\left(\mathrm{N}_{2}\right)(\mathrm{H})_{4}\left(\mathbf{6}-\mathrm{H}_{2} / \mathbf{N}_{2}\right)$ to $\left(\mathrm{P}_{6} \mathrm{ArCH}_{3}\right) \mathrm{Fe}_{2}\left(\mathrm{~N}_{2}\right)_{2}(\mathrm{H})_{4}\left(\mathbf{6}-\mathbf{N}_{2}\right)$, under $\mathrm{N}_{2}$ in a sealed $\mathbf{J}$ Young tube. 

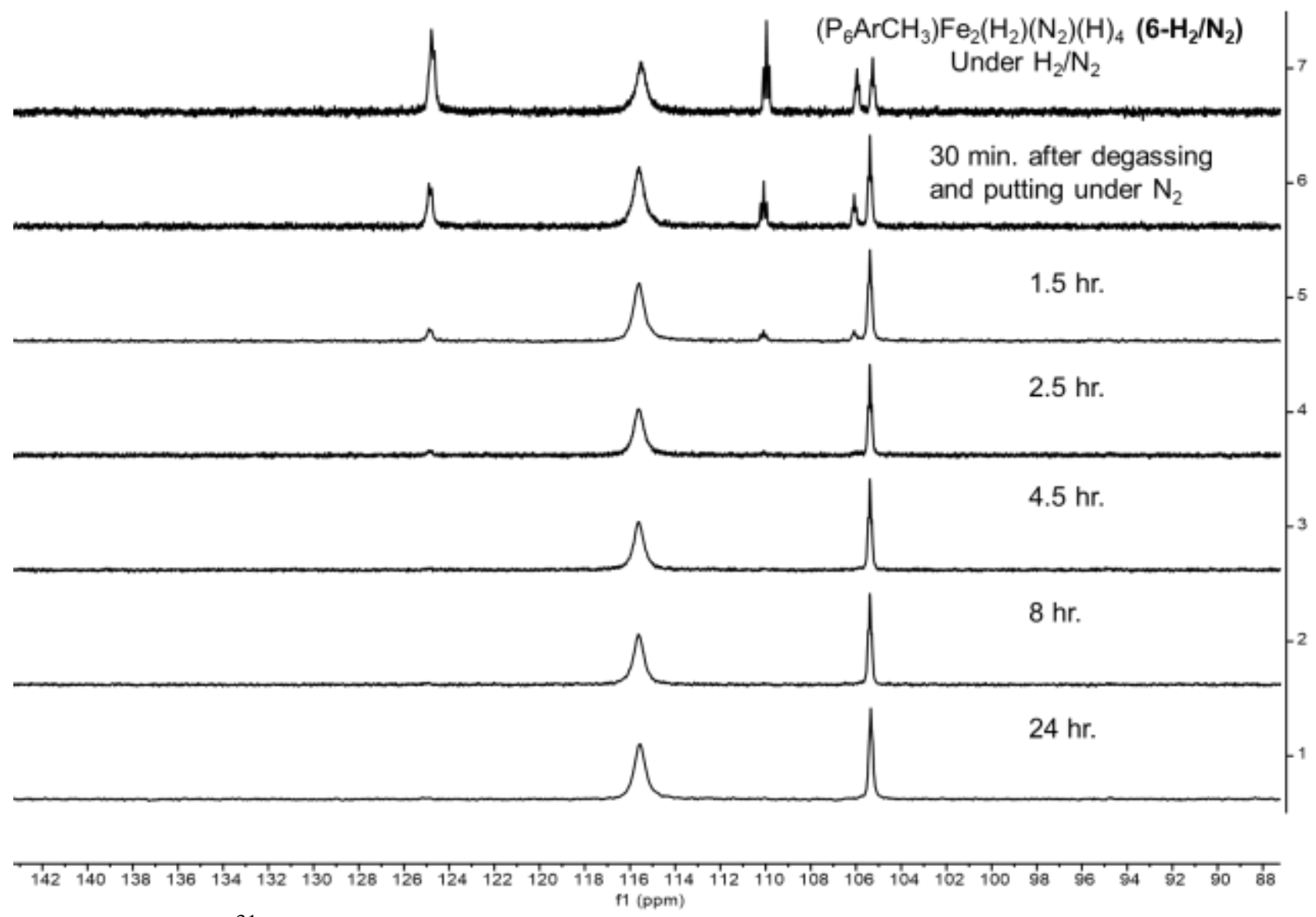

Figure S63. ${ }^{31} \mathrm{P}$ NMR spectra collected during the course of converting of $\left(\mathrm{P}_{6} \mathrm{ArCH}_{3}\right) \mathrm{Fe}_{2}\left(\mathrm{H}_{2}\right)\left(\mathrm{N}_{2}\right)(\mathrm{H})_{4}\left(\mathbf{6}-\mathrm{H}_{2} / \mathbf{N}_{2}\right)$ to $\left(\mathrm{P}_{6} \mathrm{ArCH}_{3}\right) \mathrm{Fe}_{2}\left(\mathrm{~N}_{2}\right)_{2}(\mathrm{H})_{4}\left(\mathbf{6}-\mathrm{N}_{2}\right)$, under $\mathrm{N}_{2}$ in a sealed $\mathbf{J}$ Young tube. 


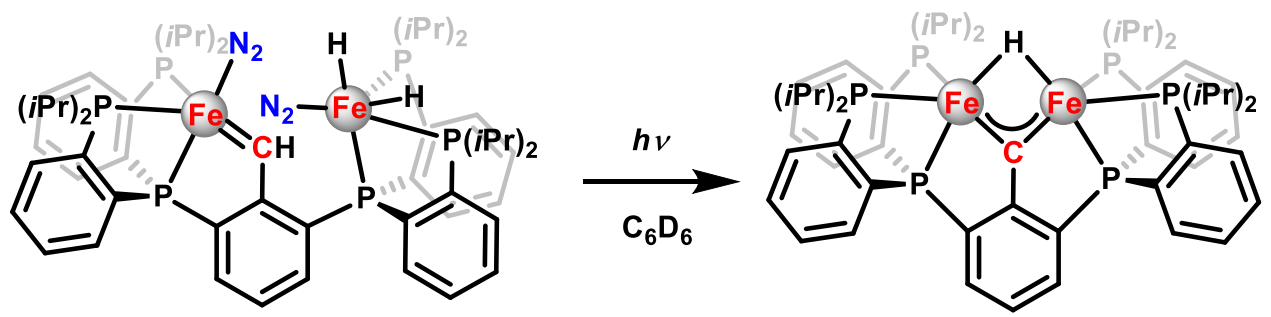

Room Temperature Photolysis of 8. A J Young NMR tube was charged with 8 (16.1 mg, 0.015 $\mathrm{mmol})$ and dissolved in $\mathrm{C}_{6} \mathrm{D}_{6}(0.45 \mathrm{~mL})$. The tube was then irradiated with $75 \mathrm{~W}$ Xe lamp and ${ }^{1} \mathrm{H}$ NMR spectra were collected at the given time points. The diagnostic ${ }^{1} \mathrm{H}$ features of $\mathbf{8}$ are consumed within $\sim 7$ hours, with concomitant formation of the paramagnetically broadened resonances of 5 .
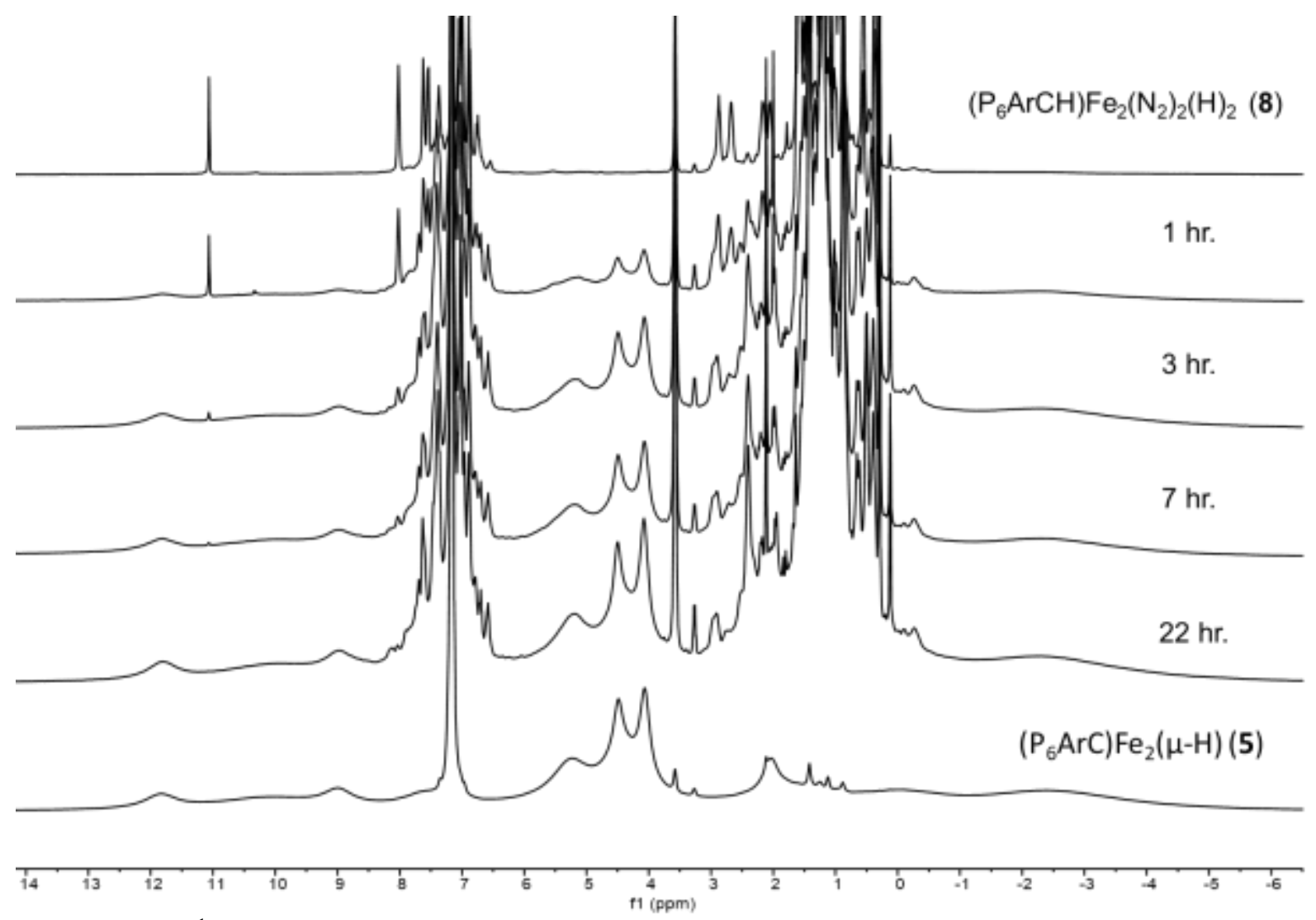

Figure S64. ${ }^{1} \mathrm{H}$ NMR spectra collected during the course of the room temperature photolysis of $\left(\mathrm{P}_{6} \mathrm{ArCH}\right) \mathrm{Fe}_{2}\left(\mathrm{~N}_{2}\right)_{2}(\mathrm{H})_{2}(\mathbf{8})$, yielding as the major product $\left(\mathrm{P}_{6} \mathrm{ArC}\right) \mathrm{Fe}_{2} \mathrm{H}(\mathbf{5})$. 


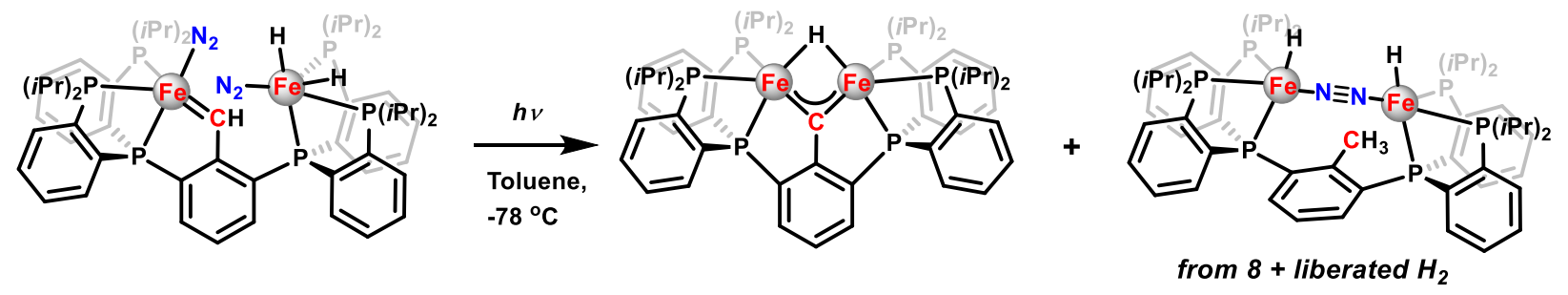

Low Temperature Photolysis of 8. A quartz Schlenk flask was charged with 8 (55.7 mg, 0.052 $\mathrm{mmol}$ ) and dissolved in toluene $(15 \mathrm{~mL})$. The flask was degassed by stirring under dynamic vacuum for 10 minutes and then chilled to $-78{ }^{\circ} \mathrm{C}$ in a dry ice/acetone bath. The chilled tube was then photolyzed from above with a $150 \mathrm{~W} \mathrm{Xe} / \mathrm{Hg}$ lamp. Aliquots were collected at the given time points for ${ }^{1} \mathrm{H}$ and ${ }^{31} \mathrm{P}$ NMR analysis. The diagnostic ${ }^{1} \mathrm{H}$ features of $\left(\mathrm{P}_{6} \mathrm{ArCH}\right) \mathrm{Fe}_{2}\left(\mathrm{~N}_{2}\right)_{2}(\mathrm{H})_{2}(\mathbf{8})$ are consumed within $\sim 7$ hours, with concomitant formation of both $\left(\mathrm{P}_{6} \mathrm{ArC}_{\mathrm{C}} \mathrm{Fe}_{2} \mathrm{H}\right.$ (5) and $\left[\mathrm{P}_{6} \mathrm{ArCH}_{3}\right](\mathrm{FeH})_{2}\left(\mu-\mathrm{N}_{2}\right)(\mathbf{7})$. The most likely origin of $\mathbf{7}$ in these experiments is from addition of $\mathrm{H}_{2}$ (liberated during the photolysis) to residual $\left(\mathrm{P}_{6} \mathrm{ArCH}\right) \mathrm{Fe}_{2}\left(\mathrm{~N}_{2}\right)_{2}(\mathrm{H})_{2}(\mathbf{8})$, rather than addition of two equivalents of $\mathrm{H}_{2}$ to $\left(\mathrm{P}_{6} \mathrm{ArC}\right) \mathrm{Fe}_{2} \mathrm{H}(\mathbf{5})$. The kinetics of addition of $\mathrm{H}_{2}$ to $\left(\mathrm{P}_{6} \mathrm{ArCH}\right) \mathrm{Fe}_{2}\left(\mathrm{~N}_{2}\right)_{2}(\mathrm{H})_{2}$ (8) have been shown to be much faster than addition to $\left(\mathrm{P}_{6} \mathrm{ArC}\right) \mathrm{Fe}_{2} \mathrm{H}(\mathbf{5})$. In fact, if $\left(\mathrm{P}_{6} \mathrm{ArC}_{2} \mathrm{Fe}_{2} \mathrm{H}\right.$ (5) is treated with $\mathrm{H}_{2} / \mathrm{N}_{2}$ at $-78{ }^{\circ} \mathrm{C}$, no reaction whatsoever is observed. Attempts to stoichiometrically add $\mathrm{H}_{2}$ to $\left(\mathrm{P}_{6} \mathrm{ArCH}\right) \mathrm{Fe}_{2}\left(\mathrm{~N}_{2}\right)_{2}(\mathrm{H})_{2}$ (8) otherwise have consistently led to overreaction to 6. However, as shown here, slow in situ generation of $\mathrm{H}_{2}$ via photolysis was a convenient strategy to observe the conversion of $\left(\mathrm{P}_{6} \mathrm{ArCH}\right) \mathrm{Fe}_{2}\left(\mathrm{~N}_{2}\right)_{2}(\mathrm{H})_{2}$ (8) to $\left[\mathrm{P}_{6} \mathrm{ArCH}_{3}\right](\mathrm{FeH})_{2}\left(\mu-\mathrm{N}_{2}\right)(\mathbf{7})$. 

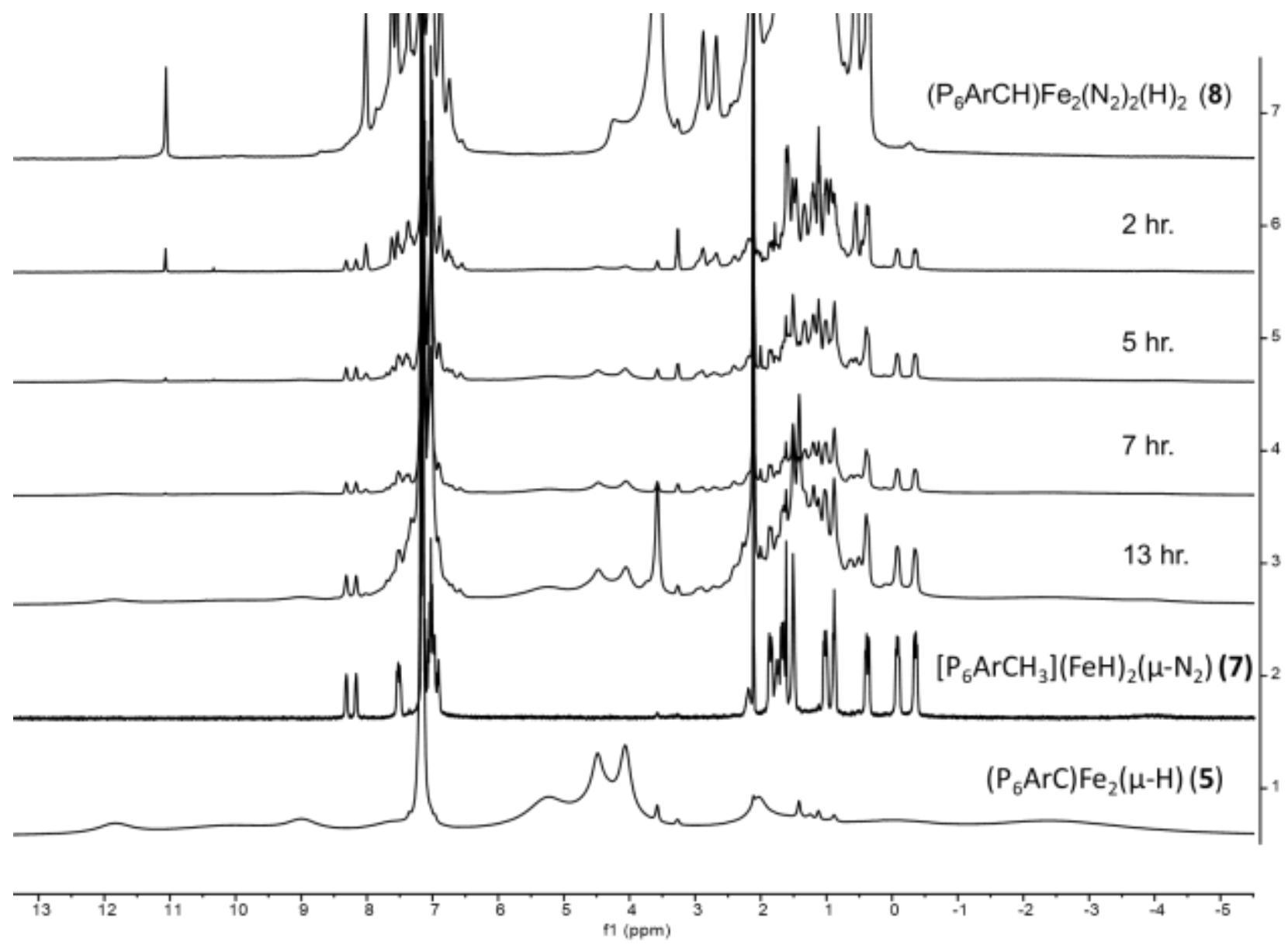

Figure S65. ${ }^{1} \mathrm{H}$ NMR spectra collected during the course of the low temperature photolysis of $\left(\mathrm{P}_{6} \mathrm{ArCH}\right) \mathrm{Fe}_{2}\left(\mathrm{~N}_{2}\right)_{2}(\mathrm{H})_{2}(\mathbf{8})$, yielding as the major diamagnetic product $\left[\mathrm{P}_{6} \mathrm{ArCH}_{3}\right](\mathrm{FeH})_{2}\left(\mu-\mathrm{N}_{2}\right)(\mathbf{7})$ as well as $\left(\mathrm{P}_{6} \mathrm{ArC}\right) \mathrm{Fe}_{2} \mathrm{H}(\mathbf{5})$. 


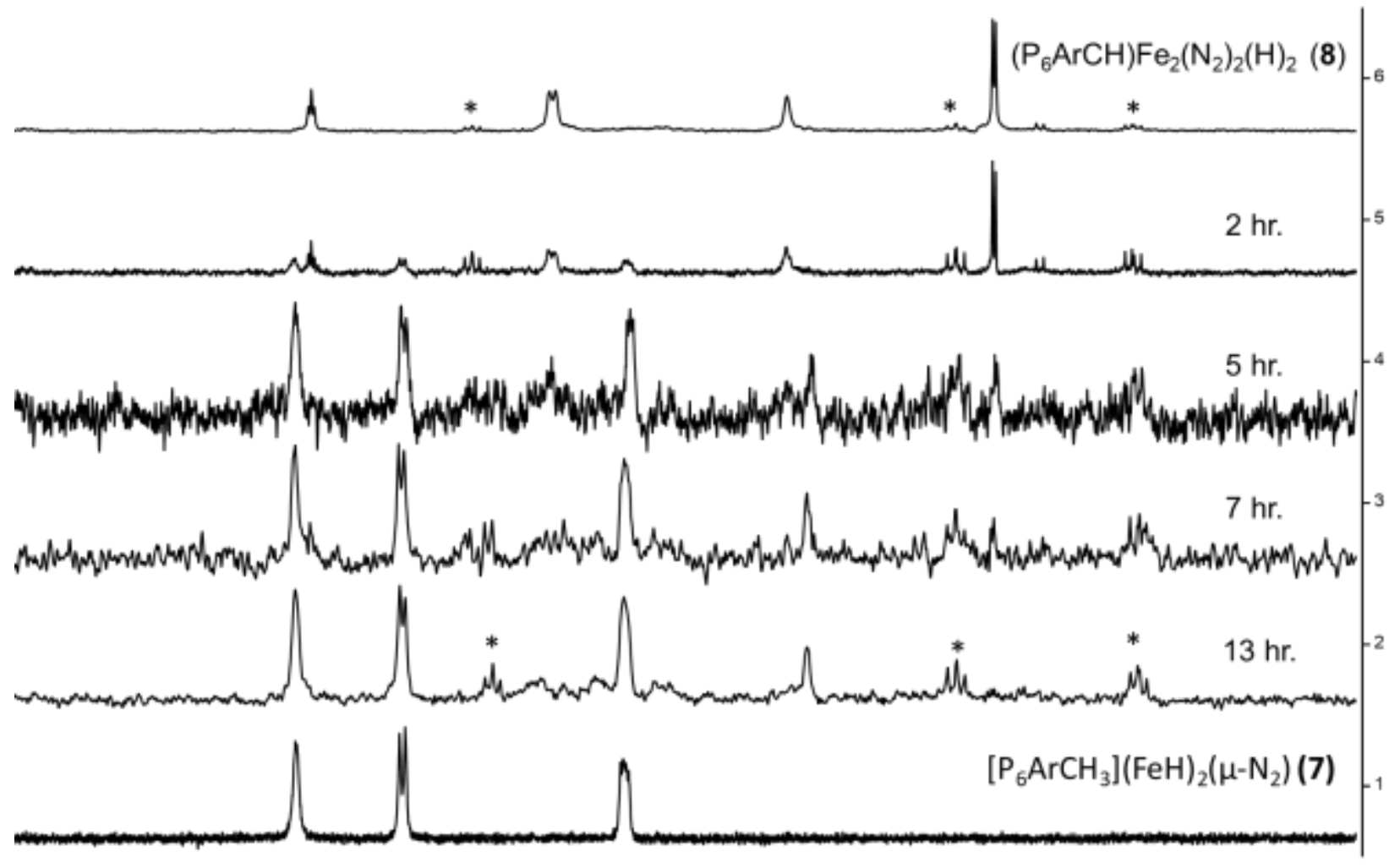

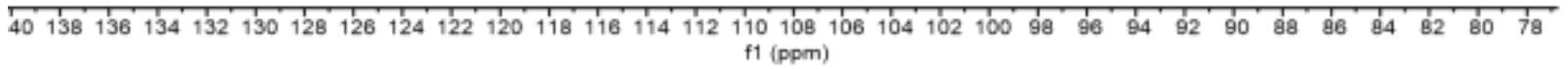

Figure S66. ${ }^{31} \mathrm{P}$ NMR spectra collected during the course of the low temperature photolysis of $\left(\mathrm{P}_{6} \mathrm{ArCH}\right) \mathrm{Fe}_{2}\left(\mathrm{~N}_{2}\right)_{2}(\mathrm{H})_{2}(\mathbf{8})$, yielding as the major diamagnetic, phosphorus containing product $\left[\mathrm{P}_{6} \mathrm{ArCH}_{3}\right](\mathrm{FeH})_{2}\left(\mu-\mathrm{N}_{2}\right)(7)$. Asterisks denote resonances originating from impurities in $\mathbf{8}$. 


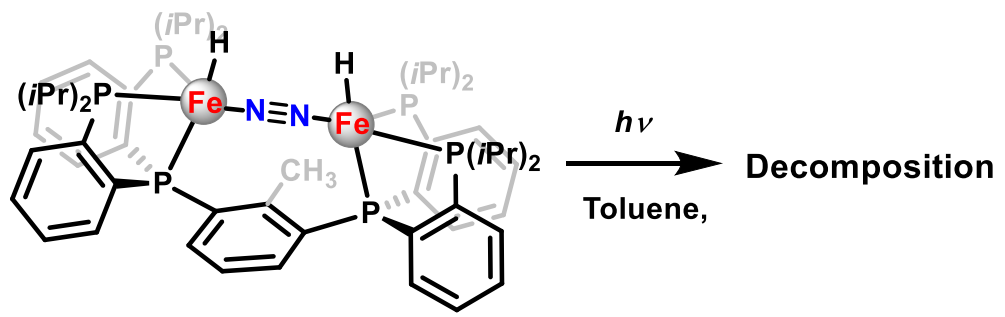

Room Temperature Photolysis of 7. A quartz Schlenk tube was charged with 7 (47.8 mg, 0.045 $\mathrm{mmol})$ and dissolved in toluene $(10 \mathrm{~mL})$. The tube was then irradiated with $75 \mathrm{~W}$ Xe lamp for 2 hours. The diagnostic ${ }^{1} \mathrm{H}$ and ${ }^{31} \mathrm{P}$ features of 7 are fully consumed, yielding an intractable mixture of products. Thus, any $\mathbf{7}$ generated during photolysis of $\mathbf{8}$ at room temperature would be rapidly decomposed.

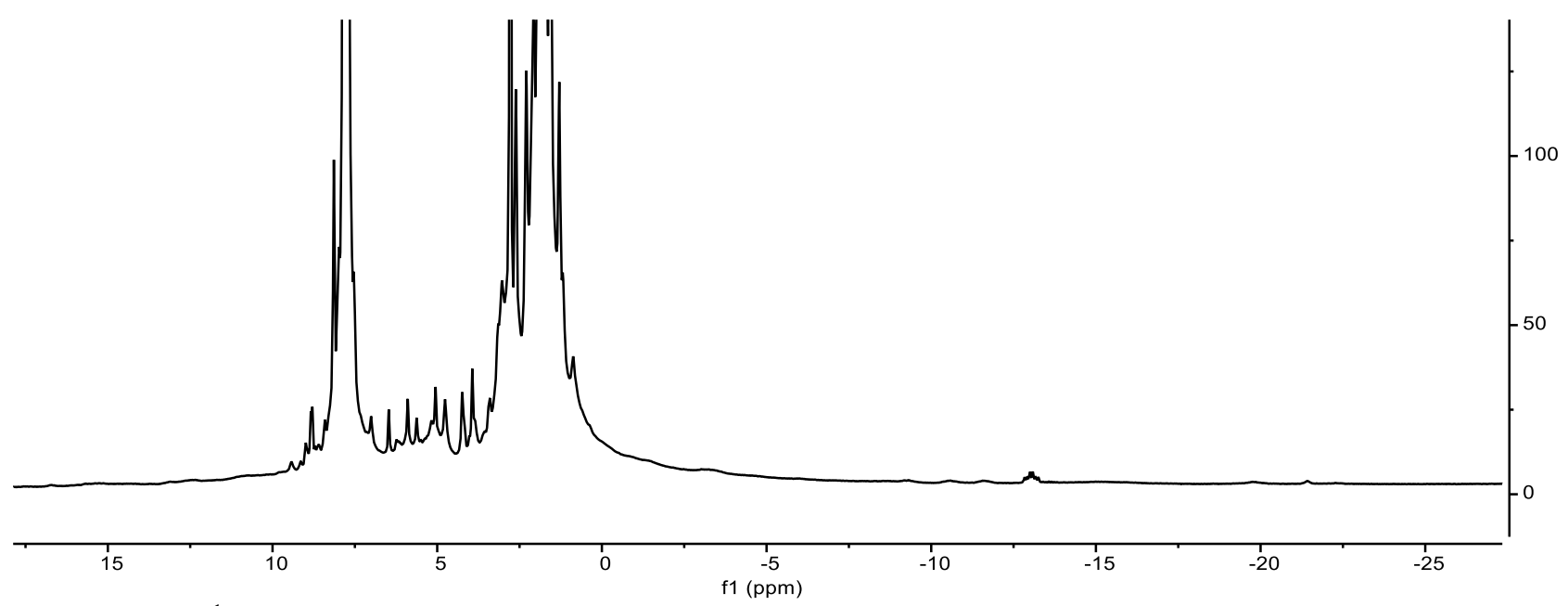

Figure S67. ${ }^{1} \mathrm{H}$ NMR spectrum collected during the course of the room temperature photolysis of $\left[\mathrm{P}_{6} \mathrm{ArCH}_{3}\right](\mathrm{FeH})_{2}\left(\mu-\mathrm{N}_{2}\right)(\mathbf{7})$, yielding an intractable mixture of products.

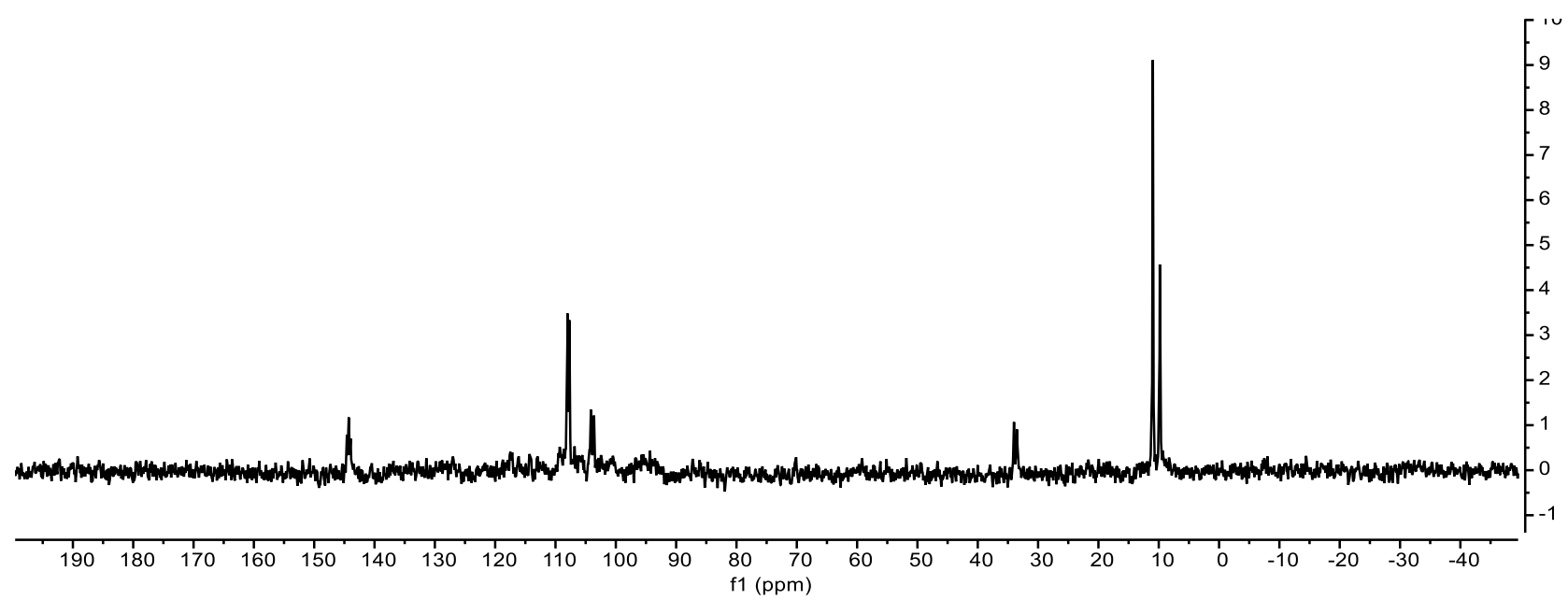

Figure S68. ${ }^{31} \mathrm{P}$ NMR spectrum collected during the course of the room temperature photolysis of $\left[\mathrm{P}_{6} \mathrm{ArCH}_{3}\right](\mathrm{FeH})_{2}\left(\mu-\mathrm{N}_{2}\right)(\mathbf{7})$, yielding an intractable mixture of products. 


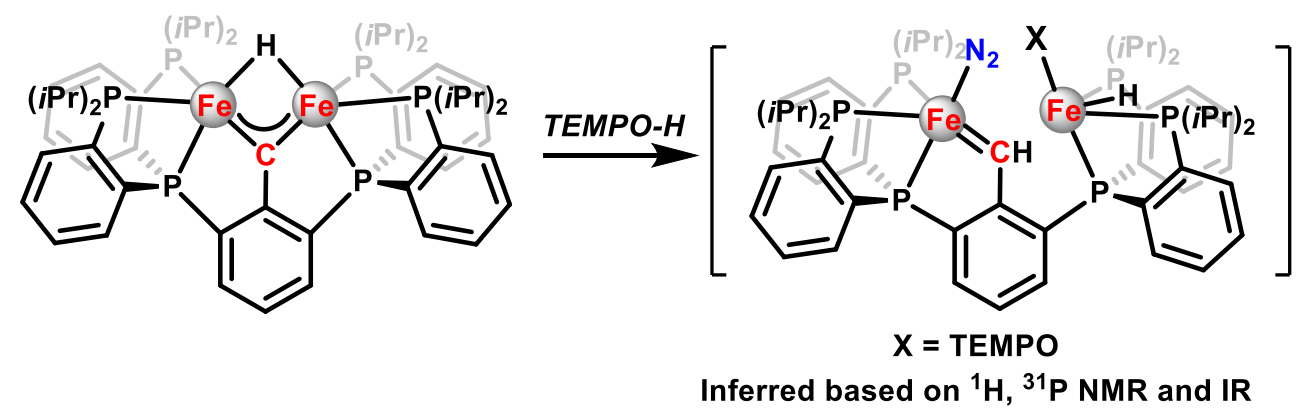

Reaction of 5 with TEMPO-H. A solution of $5(8.4 \mathrm{mg}, 0.008 \mathrm{mmol})$ in tetrahydrofuran was frozen in a glovebox cold well in a $20 \mathrm{~mL}$ scintillation vial. The vial was then removed from the cold well and as it thawed on the stir plate, a solution of TEMPO-H $(2.8 \mathrm{mg}, 0.016 \mathrm{mmol}, 2$ equiv.) was added. After 30 minutes, the mixture was concentrated to dryness and redissolved in $\mathrm{C}_{6} \mathrm{D}_{6}$ for NMR analysis. After 44 hours at room temperature, a single major phosphoruscontaining diamagnetic species was observed in the ${ }^{31} \mathrm{P}$ NMR spectrum. This species features a diagnostic carbene $\mathrm{C}-\mathrm{H}$ resonance at $11.7 \mathrm{ppm}$. The IR spectrum of this mixture exhibits a $v_{\mathrm{N} 2}$ stretch at $2076 \mathrm{~cm}^{-1}$. Although we have not been successful in isolating and crystallizing this new product in pure form, the spectroscopic data intimates a structure very similar to that of $\mathbf{8}$.

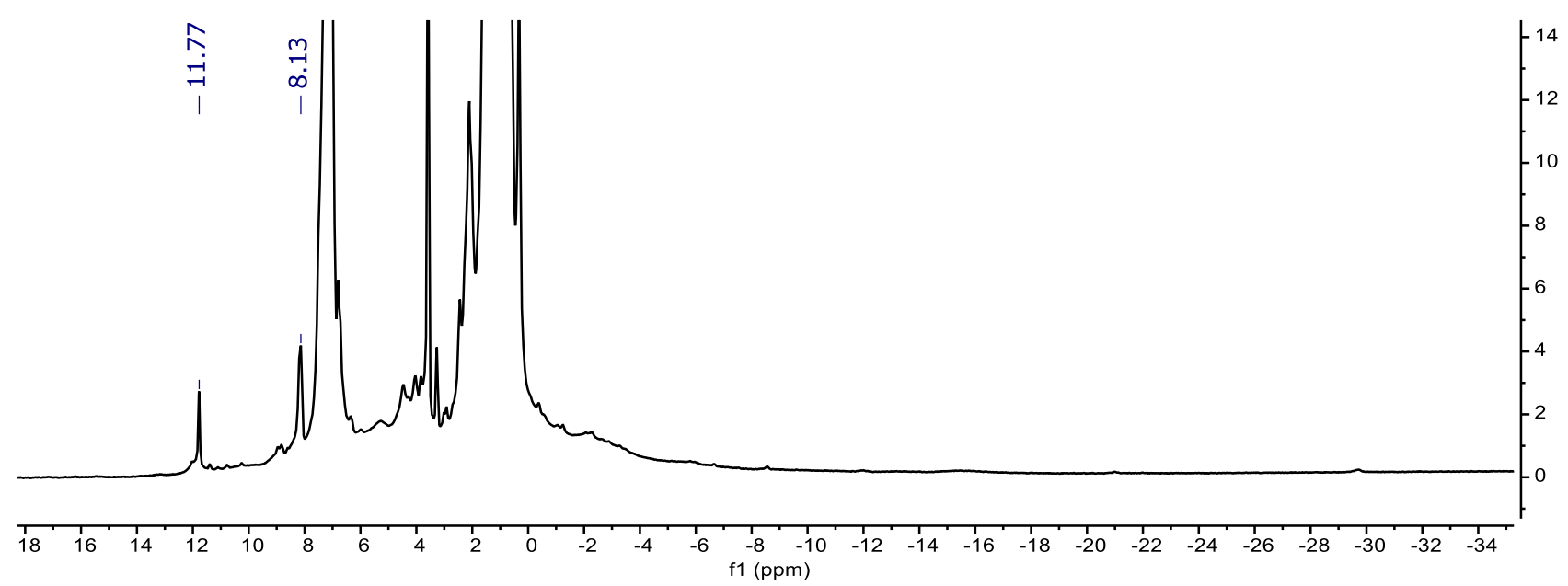

Figure S69. ${ }^{1} \mathrm{H}$ NMR spectrum following reaction of $\left(\mathrm{P}_{6} \mathrm{ArC}\right) \mathrm{Fe}_{2} \mathrm{H}(5)$ with TEMPO-H. 


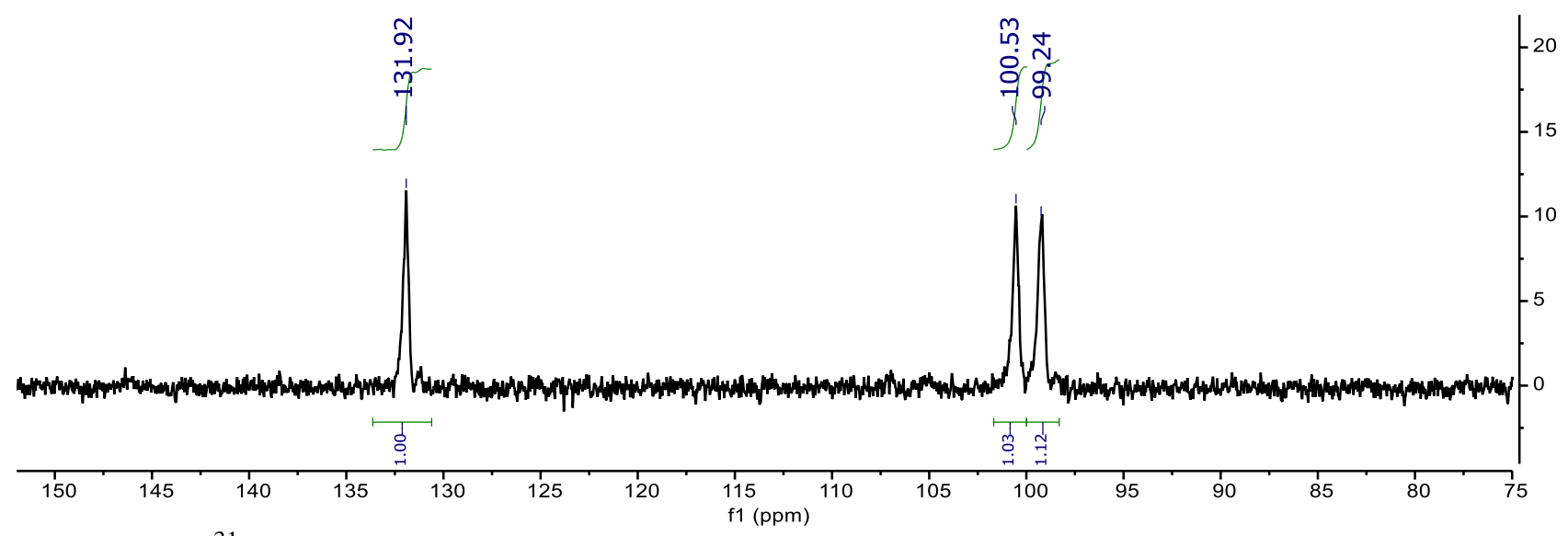

Figure S70. ${ }^{31} \mathrm{P}$ NMR spectrum following reaction of $\left(\mathrm{P}_{6} \mathrm{ArC}\right) \mathrm{Fe}_{2} \mathrm{H}(5)$ with TEMPO-H.

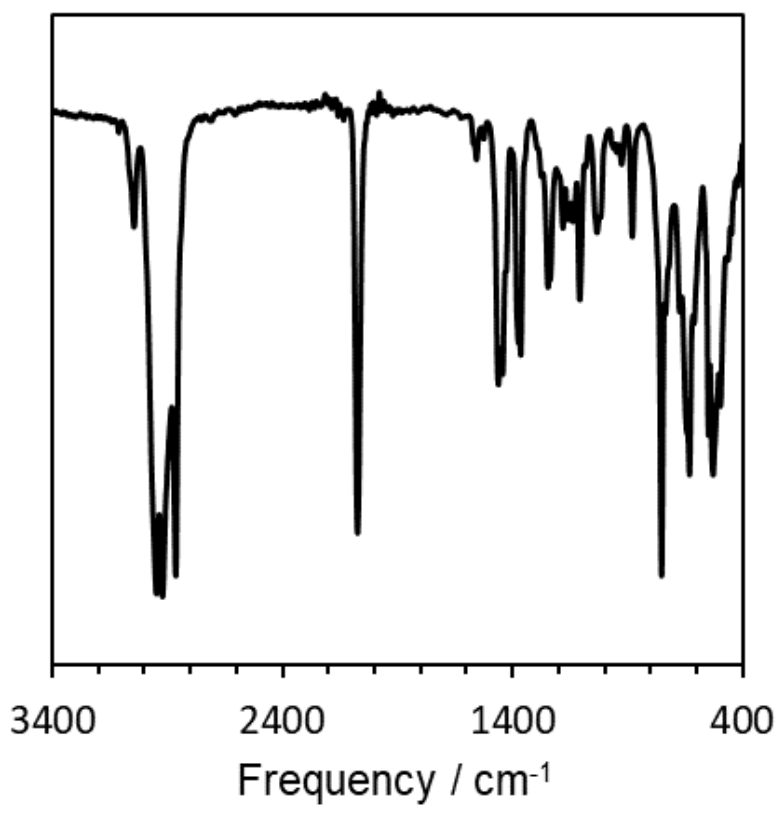

Figure S71. IR spectrum following reaction of $\left(\mathrm{P}_{6} \mathrm{ArC}\right) \mathrm{Fe}_{2} \mathrm{H}(5)$ with TEMPO-H. 


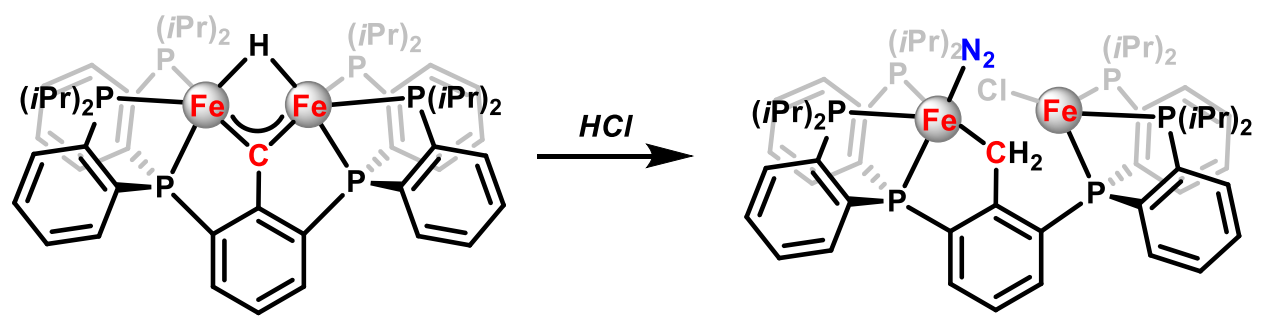

Reaction of 5 with HCl. A suspension of $5(31.9 \mathrm{mg}, 0.03 \mathrm{mmol})$ in diethyl ether $(6 \mathrm{~mL})$ was chilled to $-78^{\circ} \mathrm{C}$. A solution of anhydrous $\mathrm{HCl}$ in diethyl ether $(27 \mu \mathrm{L}, 1 \mathrm{M}, 1$ equiv. $)$ was added via microsyringe. The solution was allowed to warm to room temperature slowly overnight. The reaction mixture was concentrated to dryness. The residue was washed with pentane and collected on a bed of Celite. Material was eluted with diethyl ether (16.5 mg) and toluene $(6 \mathrm{mg})$. The major product in both fractions is the same paramagnetic iron complex. A high quality structure of this species was not obtained, but the diffraction data was sufficient to confirm its identity as an alkyliron- $\mathrm{N}_{2}$, iron-chloride species resulting from protonation at the central carbon.

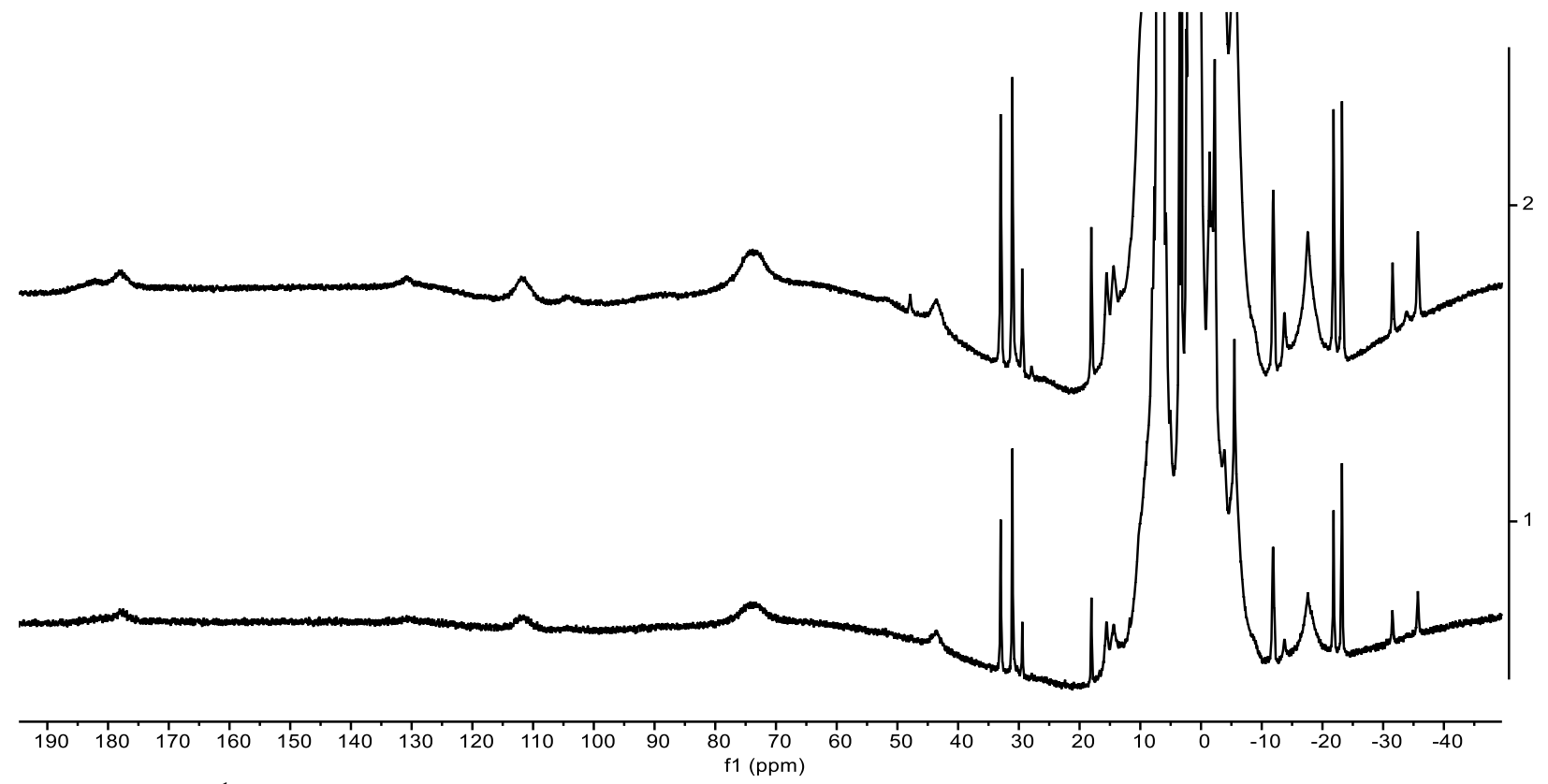

Figure S72. ${ }^{1} \mathrm{H}$ NMR spectra following reaction of $\left(\mathrm{P}_{6} \mathrm{ArC}\right) \mathrm{Fe}_{2} \mathrm{H}(5)$ with $\mathrm{HCl}$. Top: Diethyl ether fraction. Bottom: Toluene fraction. 


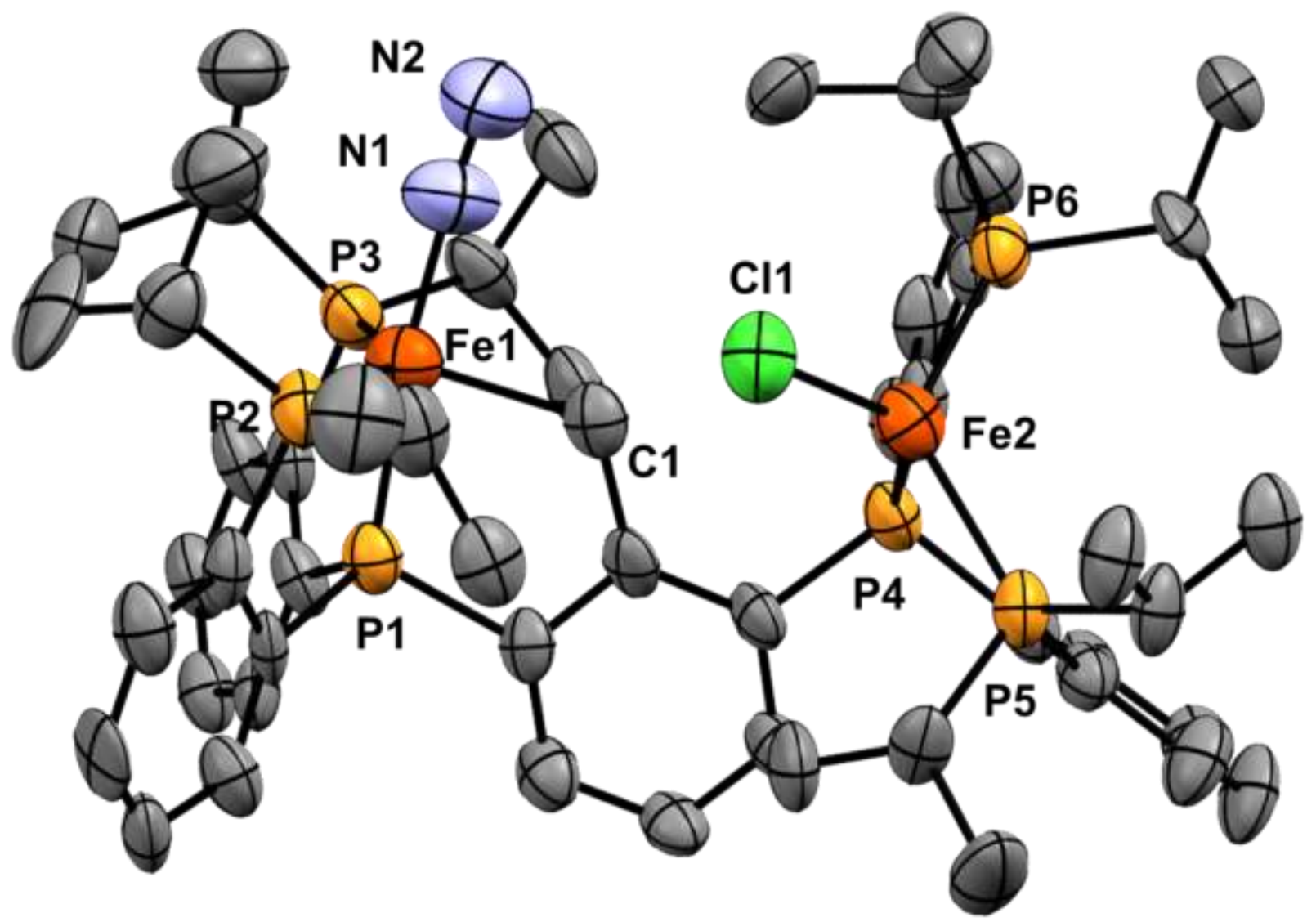

Figure S73. Crystal structure obtained from the reaction of $\left(\mathrm{P}_{6} \mathrm{ArC}_{2} \mathrm{Fe}_{2} \mathrm{H}(5)\right.$ with $\mathrm{HCl}$. Data quality is only sufficient to identify the species as $\left(\mathrm{P}_{6} \mathrm{ArCH}_{2}\right) \mathrm{Fe}\left(\mathrm{N}_{2}\right) \mathrm{FeCl}$. 


\section{DFT Calculations}

Table S1. Comparison of Experimental and Calculated Structural Metrics for $\left(\mathrm{P}_{6} \mathrm{ArC}\right) \mathrm{Fe}_{2}(\mu-\mathrm{H})$.

\begin{tabular}{|c|c|c|c|c|c|c|c|}
\hline \multicolumn{2}{|c|}{} & \multicolumn{3}{c|}{ TPSS } & \multicolumn{3}{c|}{ BP86 } \\
\hline & Exp. & $\mathrm{S}=0$ & $\mathrm{~S}=1$ & $\mathrm{~S}=2$ & $\mathrm{~S}=0$ & $\mathrm{~S}=1$ & $\mathrm{~S}=2$ \\
\hline Fe1-C1 & $1.792(1)$ & 1.785 & 1.788 & 1.837 & 1.784 & 1.787 & 1.831 \\
\hline Fe1-H1 & $1.76(3)$ & 1.755 & 1.759 & 1.739 & 1.757 & 1.759 & 1.742 \\
\hline Fe1-P1 & $2.1307(7)$ & 2.119 & 2.129 & 2.185 & 2.124 & 2.133 & 2.183 \\
\hline Fe1-P2 & $2.2627(8)$ & 2.243 & 2.262 & 2.323 & 2.251 & 2.267 & 2.321 \\
\hline Fe1-P3 & $2.2522(6)$ & 2.231 & 2.258 & 2.310 & 2.235 & 2.262 & 2.310 \\
\hline Fe-Fe & $2.6776(6)$ & 2.692 & 2.697 & 2.730 & 2.700 & 2.705 & 2.727 \\
\hline
\end{tabular}

Table S2. Single point energies for TPSS-optimized geometries of $\left(\mathrm{P}_{6} \mathrm{ArC}\right) \mathrm{Fe}_{2}(\mu-\mathrm{H})$.

\begin{tabular}{|c|c|c|c|}
\hline & \multicolumn{3}{|c|}{ Energy / $\mathrm{kJ} \mathrm{mol}^{-1}$} \\
\hline Functional & $S=0$ & $S=1$ & $S=2$ \\
\hline B3LYP & 57 & 0 & 13 \\
\hline PBE0 & 68 & 0 & 5 \\
\hline TPSSh & 53 & 0 & 46 \\
\hline
\end{tabular}

Table S3. Comparison of experimental and calculated Mössbauer parameters for $\left(\mathrm{P}_{6} \mathrm{ArC}\right) \mathrm{Fe}_{2}(\mu-$ $\mathrm{H})$.

\begin{tabular}{|c|c|c|c|c|}
\hline & Exp. & $S=0$ & $S=1$ & $S=2$ \\
\hline$\rho(0)$ & & 11820.828 & 11820.651 & 11820.318 \\
\hline$\delta / \mathrm{mm} \mathrm{s}^{-1}$ & 0.25 & 0.17 & 0.24 & 0.38 \\
\hline $\mid \Delta E_{\mathrm{Q}} / / \mathrm{mm} \mathrm{s}^{-1}$ & 0.37 & 2.68 & 0.38 & 0.63 \\
\hline
\end{tabular}



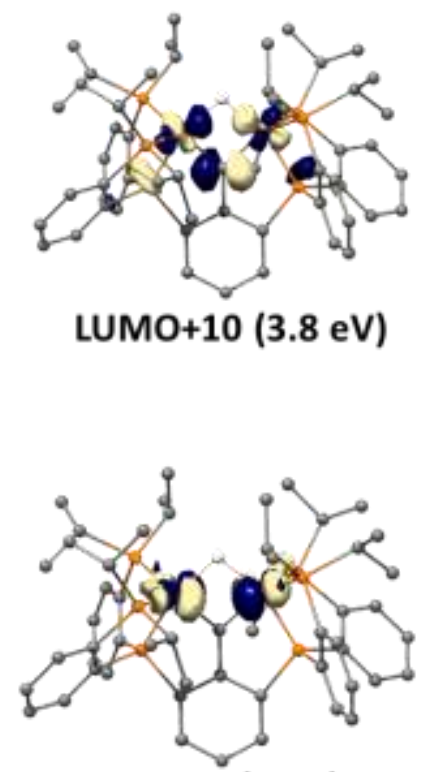

HOMO (0 eV)
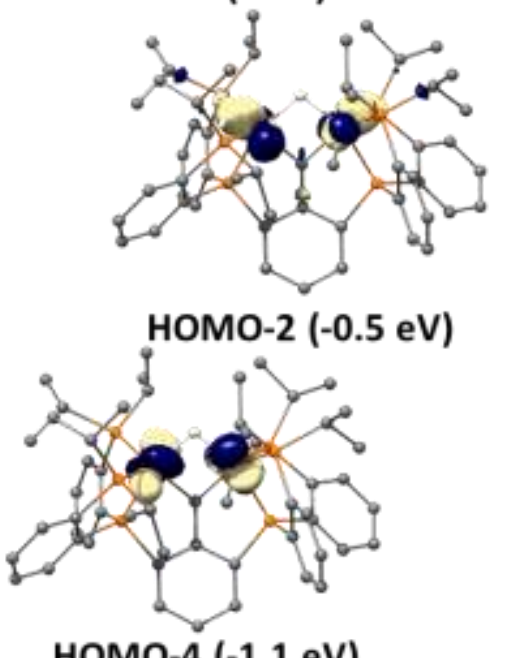

HOMO-4 (-1.1 eV) $\overline{\text { Fe-C,P }} \sigma^{*}$

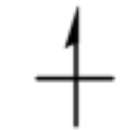

$\mathrm{Fe}-\mathrm{Fe} \pi^{*}$
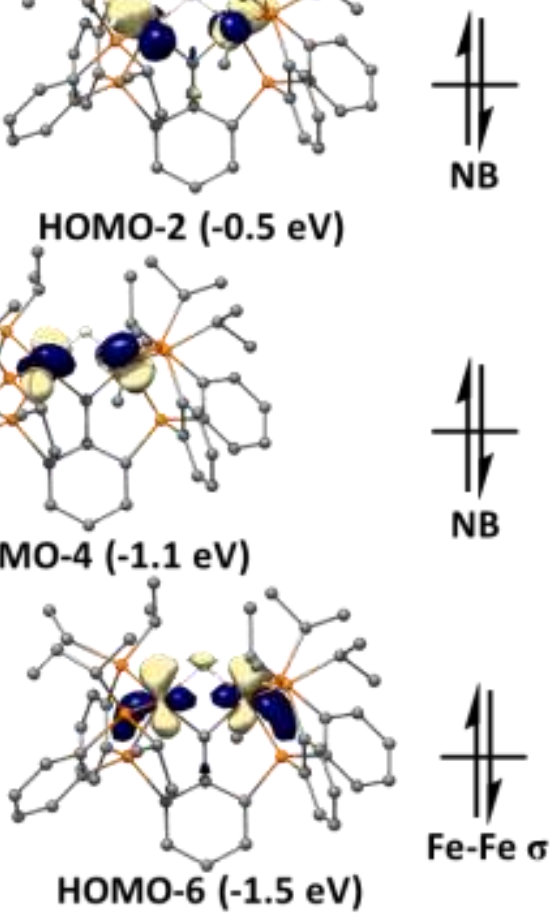

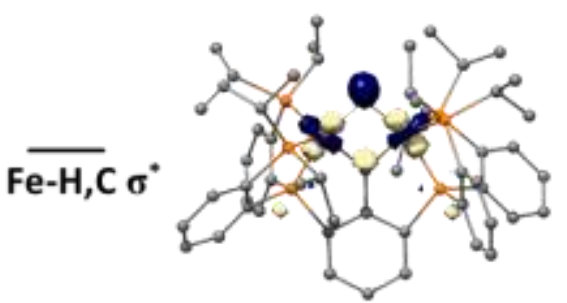

LUMO+11 (4.0 eV)
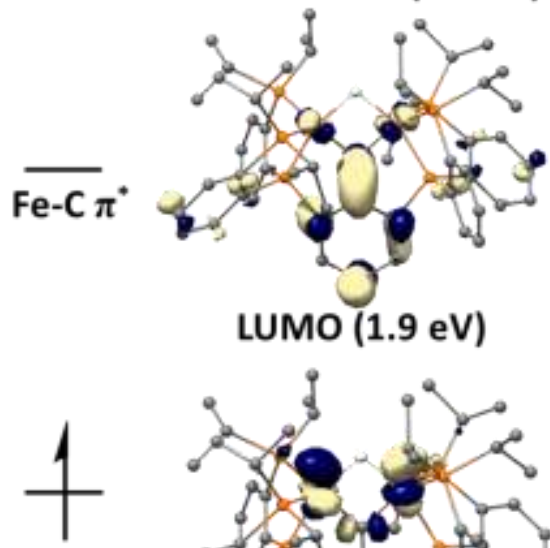

NB

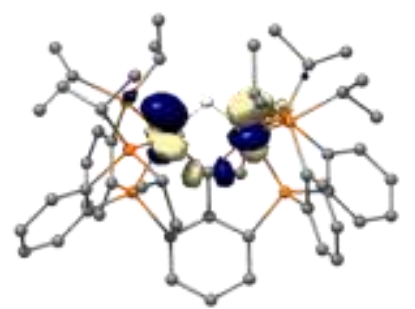

HOMO-1 (-0.1 eV)
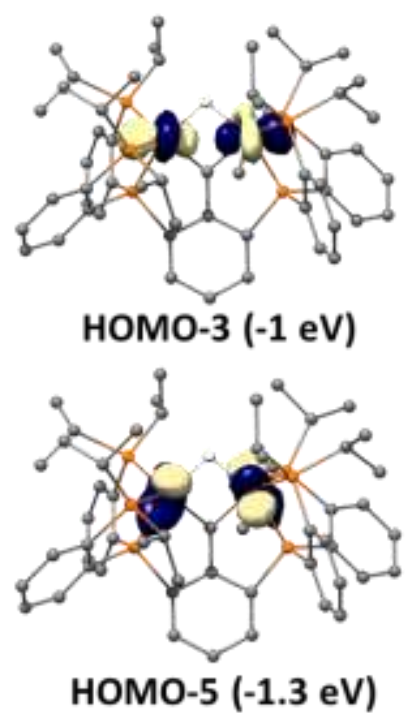

Figure S74. Calculated MO energy diagram and valence MOs for $\left(\mathrm{P}_{6} \mathrm{ArC}\right) \mathrm{Fe}_{2}(\mu-\mathrm{H})$. Isosurfaces are shown at the $0.03 \mathrm{e}^{3}$ level and orbital energies (relative to the HOMO) are provided. $\mathrm{Fe}-\mathrm{Fe}$ axial symmetry labels are a guide for the shape of the orbitals only. 


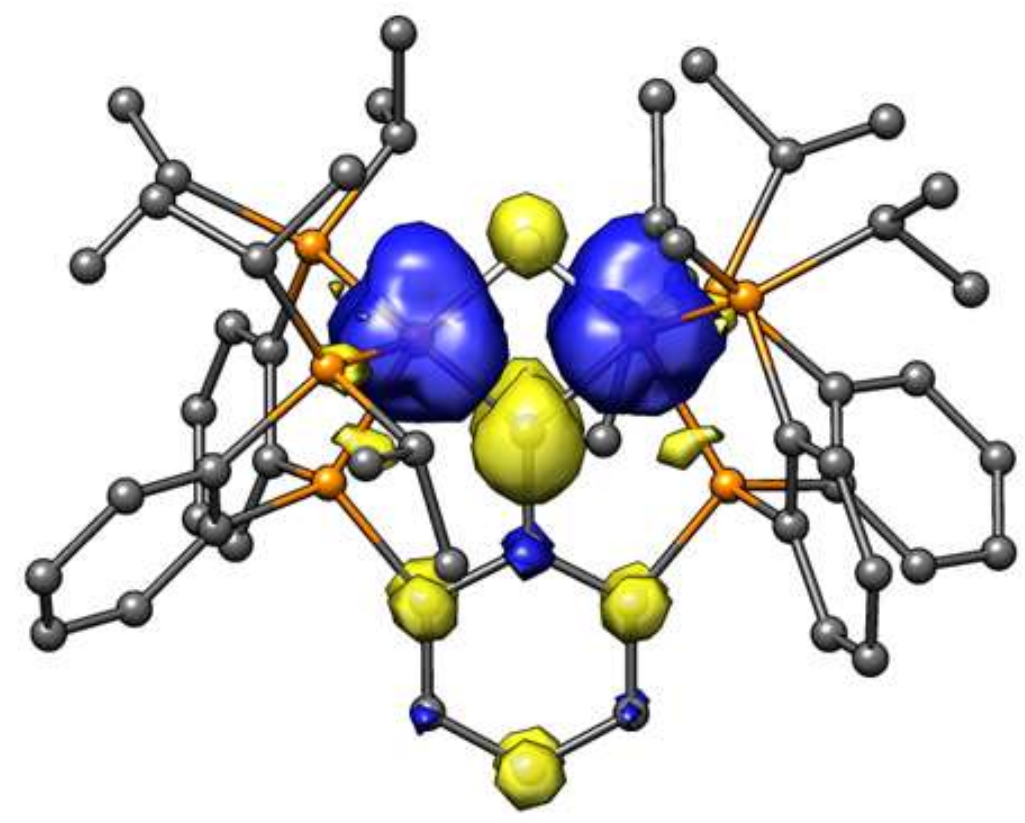

Figure S75. Spin density plot $\left(0.003\right.$ e/ $\AA^{3}$ isocontours $)$ of $\left(\mathrm{P}_{6} \mathrm{ArC}\right) \mathrm{Fe}_{2}(\mu-\mathrm{H})$.

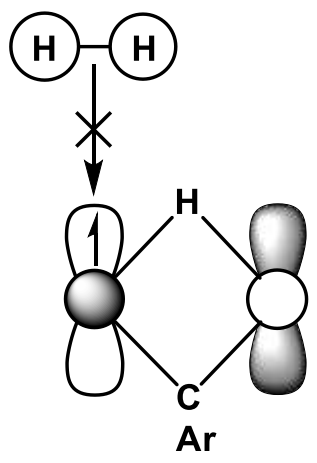

Figure S76. Illustration of the origin of spin-blocking in the activation of $\mathrm{H}_{2}$ by 5 .

Table S4. Coordinates for optimized geometry (TPSS/TZVP) of $\left(\mathrm{P}_{6} \mathrm{ArC}_{2}\right) \mathrm{Fe}_{2}(\mu-\mathrm{H})$ with $S=0$

Fe 6.97553102415853

P 7.04572223269036

P 8.33119097442988

P 4.83596777744102

C 7.37762946803575

C 5.43580347637533

H $\quad 6.24180297891960$

C 10.95662552229753

H 11.89605051931444

C 7.38075080845652
3.17947400194263

4.98382433369500

2.33259343155461

3.06338366457256

5.79332350348217

6.28053511012137

6.99607888431342

4.95485964390282

4.90778541186987

4.35164632403238
7.68808463971111

8.79379281908269

9.26045121292101

8.31348593758368

6.40332609345700

10.83991348137133

11.01077789430028

11.29183558565376

11.84579112236908

6.40433992926423 


\begin{tabular}{|c|c|c|c|}
\hline & 7.35684533543336 & 8.51694061864025 & \\
\hline & 7.34743352278283 & 9.60850552287602 & \\
\hline & 06707980960916 & 2.40958380969602 & 11.78249012579897 \\
\hline & 73549178019109 & 3.14873589764701 & 12.24705076810502 \\
\hline & & 1.85190919360505 & 12.58974716393861 \\
\hline & .30002136076308 & 2.95709813391258 & 11.22063602140102 \\
\hline & 0.41778220791321 & 3.78165955035994 & 10.73796912755552 \\
\hline & 0.95065325155378 & 2.84459286901089 & 170003 \\
\hline & & -0.208 & 3320 \\
\hline & 565 & 4888201477 & 4721 \\
\hline & & -0.820 & 4680 \\
\hline & 517 & -0.11 & 3245 \\
\hline & & & 5283 \\
\hline & 07 & 5.490 & 00131 \\
\hline & & & \\
\hline & 97 & 7.18 & 4266 \\
\hline & 9.217 & 3.81 & \\
\hline & & & 3245 \\
\hline & $9.52 ?$ & 0.00 & 8492 \\
\hline & & & 7682 \\
\hline & & & 5751 \\
\hline & & & \\
\hline & & & 3734 \\
\hline & & 4.427 & 4836 \\
\hline $\mathrm{H}$ & & & 323 \\
\hline & 5.579 & 5.26 & \\
\hline & & & 586 \\
\hline & 7.18 & & 8696 \\
\hline & & & 389 \\
\hline & 2.77 & & 020 \\
\hline & 8.550 & 5.05 & 5611 \\
\hline & 10.29 & 3678 & 22086 \\
\hline & & & \\
\hline & 11.0 & & 51020 \\
\hline & 11.5 & 4843 & \\
\hline & 11.60 & 2114 & 594460 \\
\hline & 41764879 & 0.69226865034841 & 671548 \\
\hline & 2.13484059550413 & 3.477597 & 8151364397 \\
\hline & & 2.41345225164925 & 7.20217019642051 \\
\hline & & 3.92095478550293 & \\
\hline & 35039202 & 3.96437724207358 & 8.07796005853167 \\
\hline & 3.88953 & 5.21527208190495 & 6.69318007704714 \\
\hline & 3.54220042242796 & 5.83389304233963 & 7.53356589889995 \\
\hline & 3.33710497877348 & 5.52572171221656 & \\
\hline & 4.95066934080773 & 5.42278426657166 & 6.52332949202665 \\
\hline & 3.89631011534986 & 0.45749784963525 & 7.88201766262306 \\
\hline
\end{tabular}




\begin{tabular}{|c|c|c|c|}
\hline & 3.48765913538361 & 0.77084983227919 & 6.91199069357853 \\
\hline & 3.32408694892255 & -0.42133146803328 & 8.21901938468871 \\
\hline & 4.94073020706001 & 0.15052372443854 & 7.72267173549572 \\
\hline & 7.20065470095737 & 6.43645940920034 & 7.63166085541409 \\
\hline & 4.54019176083941 & 4.32739095428660 & 9.65866950218520 \\
\hline & 3.64253917102856 & 3.72177063724556 & 6.96266575700654 \\
\hline & 3.97152622906851 & 3.16790407324013 & 6.07143739978989 \\
\hline & 9.59044696655114 & 1.18033772241854 & 8.44226117929795 \\
\hline & 9.59741183047156 & 1.62284361342706 & 7.43437921622912 \\
\hline & 7.18953724791853 & 7.83383435464 & 53627 \\
\hline & 7.045049640430 & & 16411 \\
\hline & 4.24369851436966 & 1.0490696414 & 86644 \\
\hline & 5.2672489 & & 27555 \\
\hline & 3.5771341 & 0.2233392172 & 335782 \\
\hline & 322945 & & 04838 \\
\hline & $7.3738331 \mathrm{C}$ & $2.0535616286 \mathrm{C}$ & 63691 \\
\hline & 7.7029347 & 4.9855 & 2191 \\
\hline & 6.4180406 & 2.33218542294 & 5384 \\
\hline & 9.9140760 & 3.06466436290 & 5310 \\
\hline & 9.316866 & & 30361 \\
\hline & 8.511276 & $7.0009^{\prime}$ & 78610 \\
\hline & 3.795197 & & 939 \\
\hline $\mathrm{H}$ & 2.855894 & 4.9034 & 5007 \\
\hline & 7.682461 & & 5730 \\
\hline $\mathrm{H}$ & 7.013346 & & 0365 \\
\hline & & & \\
\hline$H$ & 8.448119 & 2.9573185785 & 3616 \\
\hline & 4.33316843241092 & 3.77915184861127 & 4177 \\
\hline $\mathrm{H}$ & 3.7995978 & 2.8421 & 019 \\
\hline & 5.81246528105286 & -0.20817011151620 & 5655 \\
\hline $\mathrm{H}$ & 5.864755 & -0.7546 & 98231 \\
\hline $\mathrm{H}$ & 5.22330443477885 & -0.8199683618 & 505091 \\
\hline $\mathrm{H}$ & 6.82887143421883 & -0.11161248004959 & 80105 \\
\hline C & 11.52248237345678 & 5.44222197693348 & 764817 \\
\hline $\mathrm{H}$ & 12.43479166248392 & 5.50014521322097 & 367363 \\
\hline $\mathrm{C}$ & 5.66151943452696 & 6.22403583146048 & 34103 \\
\hline $\mathrm{H}$ & 6.16679603527583 & 7.18147129475318 & 75764 \\
\hline $\mathrm{C}$ & 5.53240672855479 & 3.80932000575575 & 2.80712441270558 \\
\hline & 5.77631993697213 & 0.76778590150054 & 42663 \\
\hline $\mathrm{H}$ & 5.22742525038470 & 0.00465354700083 & 1.66081801231095 \\
\hline & 6.23039285194008 & 0.26736504401020 & 0.22474759862232 \\
\hline $\mathrm{H}$ & 5.05596924523624 & 1.50038276849275 & 0.70613456729892 \\
\hline & 10.49042819587142 & 6.37078730399280 & 1.20561557683161 \\
\hline $\mathrm{H}$ & 10.59645899403291 & 7.15349355587504 & 0.45233511807172 \\
\hline & 11.38463955973120 & 4.43344250181060 & 2.37558359011963 \\
\hline & 12.19714902531778 & 3.71820130332438 & 2.51289075691103 \\
\hline
\end{tabular}




$\begin{array}{llcc}\text { C } & 9.17162147365322 & 5.27229686038775 & 2.93499445886729 \\ \mathrm{C} & 6.88535506135822 & 1.44548800349780 & 1.92235133349765 \\ \mathrm{H} & 7.56483258849640 & 0.65570265367479 & 2.27971407384765 \\ \mathrm{C} & 10.92480742949079 & 1.58002555201843 & 3.87054468453193 \\ \mathrm{H} & 11.96986467191183 & 1.91803068196256 & 3.79859127167271 \\ \mathrm{C} & 6.19974473642743 & 5.05475829983837 & 2.95137937721291 \\ \mathrm{C} & 4.45762794922192 & 6.17658837858775 & 1.66447879674574 \\ \mathrm{H} & 4.03748781625987 & 7.08914867179862 & 1.23792217519666 \\ \mathrm{C} & 3.70187840092540 & 1.08619695660387 & 3.87436104237885 \\ \mathrm{H} & 3.19361015440149 & 2.05848761730092 & 3.91407200134852 \\ \mathrm{H} & 3.14577772003611 & 0.39843360450265 & 4.53225337797438 \\ \mathrm{H} & 3.61467692741752 & 0.69231961441497 & 2.85288555793155 \\ \mathrm{C} & 12.61432555649874 & 3.46374004826038 & 5.65105824457001 \\ \mathrm{H} & 12.87469907485291 & 2.39768011179375 & 5.61592317852731 \\ \mathrm{H} & 13.16358001950566 & 3.90511739620902 & 6.49819325717431 \\ \mathrm{H} & 12.99263896503901 & 3.94587356060610 & 4.73722042563087 \\ \mathrm{C} & 10.87036664583937 & 5.21507578875780 & 6.10945359578376 \\ \mathrm{H} & 11.22768659485379 & 5.82881467207737 & 5.26967081153198 \\ \mathrm{H} & 11.41948961153601 & 5.52352789791438 & 7.01255086962801 \\ \mathrm{H} & 9.80985423277765 & 5.43105545986019 & 6.27235058800444 \\ \mathrm{C} & 10.84398590703564 & 0.45441947332474 & 4.91680177410871 \\ \mathrm{H} & 11.24867334316409 & 0.76323885452731 & 5.88995001935349 \\ \mathrm{H} & 11.41578124029064 & -0.42449628969300 & 4.57926997014384 \\ \mathrm{H} & 9.79811785615846 & 0.14937176735505 & 5.07025859835524 \\ \mathrm{C} & 7.54414930082297 & 6.43801736258419 & 5.17379250725754 \\ \mathrm{C} & 10.21116972913700 & 4.33086787612159 & 3.14973672773239 \\ \mathrm{C} & 11.10754574937683 & 3.71898255839236 & 5.84523252730242 \\ \mathrm{H} & 10.77119578827542 & 3.17020504220516 & 6.73682804022933 \\ \mathrm{C} & 5.15807774040088 & 1.18090616713601 & 4.36378277937926 \\ \mathrm{H} & 5.15041204079570 & 1.62410529133275 & 5.37137790644761 \\ \mathrm{C} & 7.53261282423515 & 7.83833881589095 & 5.17968183678147 \\ \mathrm{H} & 7.66165349201200 & 8.41903607127764 & 4.26813844775882 \\ \mathrm{C} & 10.50883446000183 & 1.05619513463751 & 2.48939223086463 \\ \mathrm{H} & 9.48449763045968 & 0.66397451502471 & 2.50900677885105 \\ \mathrm{H} & 11.17516188880838 & 0.23043138417768 & 2.19247545390718 \\ \mathrm{H} & 10.56590994181147 & 1.83084578513772 & 1.71411374300931 \\ \mathrm{Fe} & 7.77513154955421 & 3.17967659431600 & 5.11754498898015\end{array}$

Table S5. Coordinates for optimized geometry (TPSS/TZVP) of $\left(\mathrm{P}_{6} \mathrm{ArC}_{2} \mathrm{Fe}_{2}(\mu-\mathrm{H})\right.$ with $S=1$
Fe 6.96116170639783
3.14301034356543
7.68621779847019
P 7.00968356494003
4.95909228331065
8.79243149553237
P 8.36588549365855
2.34653500047894
9.26896072042439
P 4.79085044319016
3.04044885104220
8.29860605516874
C 7.37557546637513
5.75891513029622
6.40324976901339
C 5.40598301437459
6.23699521890766
10.85049779406735 


\begin{tabular}{|c|c|c|c|}
\hline & 6.20894538540367 & & \\
\hline & 10.92430070022542 & .03327203162866 & 11.28948103876886 \\
\hline & 11.86400673771541 & & 11.84455622655799 \\
\hline & 37665071594690 & 4.31660175998190 & 6.40364955635056 \\
\hline & & & \\
\hline & 35793046266598 & 9.57688941836909 & 230 \\
\hline & & & 727 \\
\hline & 7.71886326255845 & 3.171873762144 & 12.2409 \\
\hline & 6.57578429793769 & & 307 \\
\hline & 6.29938046886764 & 2.94021835 & 11.2002 \\
\hline & 10.41638978752552 & $3.845246^{\circ}$ & 10.738 \\
\hline & 10.97279782656807 & 2.9222940 & 50001 \\
\hline & 9.00688330567149 & -0.19269635513767 & 51820201 \\
\hline & 8.95479 & -0.728 & \\
\hline & 9.60793 & -0.8055573 & 7.6335 \\
\hline & 7.9919 & -0.11 & \\
\hline & 3.2213 & 5.3642 & 11.4 \\
\hline & 2.321 & & \\
\hline & 9.027 & 6.254 & 683 \\
\hline & 8.500 & & 541 \\
\hline & 9.2160 & & \\
\hline & 9.0128 & $0.8145 c$ & 11.7 \\
\hline & & & \\
\hline & 8.562 & 0.31 & 164 \\
\hline & 9.716 & & 687 \\
\hline & 4.245 & 6.302 & 829 \\
\hline & 4.146 & 7.07454 & 975 \\
\hline & & & \\
\hline & & 3.646 & 509 \\
\hline & & & \\
\hline & 7.898 & & \\
\hline & & & \\
\hline & 3.7909 & & \\
\hline & 2.754 & 1.89072 & 521 \\
\hline & 8.516 & 5.0680962 & \\
\hline & $10.231<$ & 6.2405 & 11. \\
\hline & 9313380 & 7.1659978 & 466245577 \\
\hline & & & \\
\hline & 27642049 & 2.109922904 & 4467 \\
\hline & 11.6660 & 0.444135 & 466320676 \\
\hline & 014361497 & 0.75052674139481 & 3055354515 \\
\hline & 2.065 & 3.5522865 & \\
\hline & 1.74839591347815 & 2.50592403167651 & 7.36021100255123 \\
\hline & & 3.98253358186 & \\
\hline & 1.76351387903602 & 4.09958454015387 & 8.16239239365868 \\
\hline & 3.88772200127792 & 5.17303986414361 & 6.63611129605212 \\
\hline
\end{tabular}




\begin{tabular}{|c|c|c|c|}
\hline & 3.63989510129601 & 5.84019131031272 & 7.47484974654745 \\
\hline & 3.29198318238589 & 5.48896931167264 & 5.76566117208895 \\
\hline & 4.94516751660807 & 5.30512195760462 & 6.38629473406338 \\
\hline & 3.80233061446247 & 0.45823207716911 & 7.82816153153938 \\
\hline & 3.34711356461880 & 0.80233198580503 & 6.88969743234887 \\
\hline & 3.24075557119183 & -0.42625908241421 & 8.16821851168223 \\
\hline & 4.83449119762701 & 0.14709136056285 & 7.60839180606723 \\
\hline & 7.16715261700209 & 6.40410742989024 & 7.62569795945010 \\
\hline & 4.50785134644425 & 4.28920427955600 & 9.66094447352356 \\
\hline & 3.57211660424026 & 3.70823575559277 & 6.97825312045173 \\
\hline & 3.82752065060814 & 3.10195891496325 & 6.09651157449928 \\
\hline & 9.64390684918714 & 1.20581341661428 & 8.46666573401122 \\
\hline & 9.65172831339537 & 1.64064421186103 & 7.45507279184509 \\
\hline & 7.15749109313478 & 7.80125018857197 & 7.62325626255886 \\
\hline $\mathrm{H}$ & 6.98895536606645 & 8.38013395997325 & 8.53359303710606 \\
\hline $\mathrm{C}$ & 4.28423692306846 & 0.97775157087725 & 10.24989575935503 \\
\hline $\mathrm{H}$ & 5.31111881566921 & 0.60106661943148 & 10.15907941256221 \\
\hline $\mathrm{H}$ & 3.64399701872201 & 44162695 & 225206014019 \\
\hline & 4.26117491401993 & 220906871 & 11.05451188032663 \\
\hline $\mathrm{H}$ & 7.37391592873770 & 89077587 & 380 \\
\hline & 7.73893833909785 & 4.9610 & 4.01 \\
\hline $\mathrm{P}$ & 6.38292746133222 & 1614084 & 515 \\
\hline $\mathrm{P}$ & 9.95928117575199 & 3.04101831910822 & 4.50749023143318 \\
\hline $\mathrm{C}$ & 9.34572897296351 & & \\
\hline $\mathrm{H}$ & 8.54341493059793 & 2162952 & 1.7908 \\
\hline & 3.82649347941463 & 0484997 & 1.51150 \\
\hline $\mathrm{H}$ & 2.88625507513926 & 539 & 877 \\
\hline & 7.67613230833248 & 2.41340522953155 & 366601345 \\
\hline $\mathrm{H}$ & 7.02788600507057 & 8934 & 324 \\
\hline $\mathrm{H}$ & 8.17326926227235 & 2513439 & 0.22 \\
\hline $\mathrm{H}$ & 8.44757219092600 & 2.94138357905774 & 1.60390078302457 \\
\hline $\mathrm{C}$ & 4.33288528043598 & 3.84474827730000 & 2.06554678709671 \\
\hline $\mathrm{H}$ & 3.77469157690494 & & \\
\hline $\mathrm{C}$ & 5.74100457588058 & -0.19137155074044 & 4.48368063533411 \\
\hline $\mathrm{H}$ & 5.79315702961541 & -0.72772281111445 & 3.52520236522425 \\
\hline $\mathrm{H}$ & 5.13965407056912 & -0.80391547205146 & 5.17273235334270 \\
\hline $\mathrm{H}$ & 6.75591205853808 & -0.11338217847513 & 4.89902329299293 \\
\hline $\mathrm{C}$ & 11.53058821602110 & 5.36693894131528 & 1.36419019461210 \\
\hline $\mathrm{H}$ & 12.43092242515924 & 5.40589017812271 & 0.74809944808396 \\
\hline $\mathrm{C}$ & 5.72614703059286 & 6.25233534260091 & 2.39006172264215 \\
\hline $\mathrm{H}$ & 6.25402824783734 & 7.19669252612014 & 2.53098462625804 \\
\hline $\mathrm{C}$ & 5.53367738678520 & 3.84198472957645 & 2.80634064342035 \\
\hline $\mathrm{C}$ & 5.73632748613303 & 0.81198186540244 & 1.07095667710558 \\
\hline $\mathrm{H}$ & 5.16934539973400 & 0.05310551465022 & 1.62375086848248 \\
\hline $\mathrm{H}$ & 6.18744799879338 & 0.31247737531138 & 0.19774443336529 \\
\hline & 5.03217073560166 & 1.56179585743527 & 0.68401241897567 \\
\hline
\end{tabular}




\begin{tabular}{|c|c|c|c|}
\hline $\mathrm{C}$ & & & \\
\hline $\mathrm{H}$ & & & \\
\hline & 11.40077522879638 & 4.37156106783767 & 2.34664675863342 \\
\hline & 12.20767269178249 & 3.64846266526945 & \\
\hline & 9.20701624987876 & 5.23809514142553 & \\
\hline & 6.85086159581218 & & \\
\hline & 7.51275275217580 & & 1707 \\
\hline & 10.95818 & 608094 & \\
\hline & & 89419 & \\
\hline & 6.234232 & 5.067 & 2.9554 \\
\hline & & & \\
\hline & 4.12 & 78 & 1.2 \\
\hline & & & \\
\hline & 3.15 & & \\
\hline & & & \\
\hline & 3.56403 & 0.752 & 2.8153 \\
\hline & 12.6858 & 1969 & \\
\hline & & & \\
\hline & 13.2 & 3.9 & \\
\hline $\mathrm{H}$ & & & \\
\hline & 10.8 & & 64 \\
\hline & & & \\
\hline & 11.4 & & 900 \\
\hline $\mathrm{H}$ & & & \\
\hline & & & 406 \\
\hline $\mathrm{H}$ & 11.3 & & \\
\hline $\mathrm{H}$ & & 366 & \\
\hline $\mathrm{H}$ & 9.91156 & & \\
\hline $\mathrm{C}$ & 7.57 & 45 & 40 \\
\hline & 10.2 & 20368 & 066 \\
\hline $\mathrm{C}$ & 11.1793 & 6304 & 2773 \\
\hline $\mathrm{H}$ & 10.9221 & 15921 & 5052 \\
\hline $\mathrm{C}$ & & & \\
\hline $\mathrm{H}$ & 5.09 & & \\
\hline $\mathrm{C}$ & 7.57 & & \\
\hline $\mathrm{H}$ & 7.733598 & 8.386 & 4.27985 \\
\hline & & & \\
\hline $\mathrm{H}$ & 9.43913813410430 & 0.60392573984602 & 2.64234944260783 \\
\hline$U$ & & & \\
\hline $\mathrm{H}$ & 10.49133151189960 & 1.72720996469932 & \\
\hline & 7.78844918223151 & 3.14296311718149 & 5.11964567382174 \\
\hline
\end{tabular}

Table S6. Coordinates for optimized geometry (TPSS/TZVP) of $\left(\mathrm{P}_{6} \operatorname{ArC}_{2} \mathrm{Fe}_{2}(\mu-\mathrm{H})\right.$ with $S=2$

Fe 6.91798374726708 P 6.98393390555930
3.03601117732227

4.92133150969001
7.68912141028414

8.78306499570589 


\begin{tabular}{|c|c|c|c|}
\hline & & & \\
\hline & 4.67450804006340 & & \\
\hline & & & \\
\hline & 5.47338969783560 & 6.15201290150490 & \\
\hline & 6.30034707719089 & & \\
\hline & 10.88557027682860 & 765869367 & \\
\hline & 11.80795039912002 & & \\
\hline & 7.39131560037892 & 4.26896419582000 & \\
\hline & & & \\
\hline & 0242 & 673 & \\
\hline & 7.05 & & 099 \\
\hline & 7.7005019652 & 3.11 & 097 \\
\hline & 6.55621468 & & 12.5 \\
\hline $\mathrm{H}$ & 6.290 & 2.88 & \\
\hline & 07489 & 63889 & 1353 \\
\hline & 10.9 & 692 & \\
\hline & 9.07 & 1369 & \\
\hline $\mathrm{H}$ & & & \\
\hline & 9.7 & -0.7 & \\
\hline & 8.0 & & \\
\hline & 3.291 & & \\
\hline $\mathrm{H}$ & 2.417 & & \\
\hline 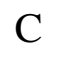 & & & \\
\hline $\mathrm{I}$ & 8.4 & & \\
\hline $\mathrm{C}$ & & & \\
\hline $\mathrm{C}$ & 8.9 & 0.7 & 600 \\
\hline $\mathrm{H}$ & & -0.0 & 892 \\
\hline & & & \\
\hline $\mathrm{I}$ & & & \\
\hline $\mathrm{C}$ & & & \\
\hline $\mathrm{H}$ & 4.303 & & \\
\hline $\mathrm{C}$ & & & \\
\hline $\mathrm{H}$ & 2.52 & & \\
\hline $\mathrm{C}$ & 5.55 & & \\
\hline $\mathrm{C}$ & & & \\
\hline $\mathrm{H}$ & 7.22 & 6068 & 10.5 \\
\hline $\mathrm{C}$ & 3.66 & & \\
\hline $\mathrm{H}$ & & & \\
\hline $\mathrm{C}$ & 8.50 & 490081633 & 9.81 \\
\hline $\mathrm{C}$ & 10.1 & & \\
\hline $\mathrm{H}$ & 061914 & 184377 & 11.4833793 \\
\hline $\mathrm{C}$ & & & \\
\hline $\mathrm{H}$ & & 2.10337080688902 & 9.03450775343997 \\
\hline & & & \\
\hline $\mathrm{H}$ & 11.20226880798591 & & 10.06427164058598 \\
\hline & 1.98036364662134 & 3.79043704785601 & 7.37410977317675 \\
\hline
\end{tabular}




\begin{tabular}{|c|c|c|c|}
\hline & 1.58130648822474 & 2.78211269788889 & 7.55010879106134 \\
\hline & 1.40466558974624 & 4.22856157526340 & 6.54322050516277 \\
\hline & 1.78327970261683 & 4.40446378418768 & 8.26481406242219 \\
\hline & 3.92008441178612 & 5.21386157532167 & 6.60676783923298 \\
\hline & .80249941057657 & 5.91933394778675 & 7.44212348657446 \\
\hline & 30173344570550 & 5.57467628939189 & 5.77034305029742 \\
\hline & 96830269234533 & 5.22545471280613 & 6.28737003829667 \\
\hline & 3.55768579580926 & 0.54855696549832 & 7.74636700662636 \\
\hline & 3.05700152608592 & 0.96053488125742 & 6.86000647724269 \\
\hline & 2.97783808097677 & -0.32553337308195 & 8.08208600972175 \\
\hline & 4.55538128435033 & 0.19957436735465 & 5166829 \\
\hline & 7.12588050653456 & 6.34611417320427 & 7.62491195296929 \\
\hline & 4.4760057012 & 4.27542899877870 & 2632211 \\
\hline & 3.47585082447892 & 3.79882595655275 & 7.00886616573437 \\
\hline & 3.62766422657569 & & \\
\hline & 9.70560202939869 & 1.20749624199647 & 8.50710556297469 \\
\hline & 9.74332097451448 & 1.67571402731020 & 7.51069009594188 \\
\hline & 7.082 & & 274953 \\
\hline & 79305 & 03602 & 860065 \\
\hline & 06940 & 0.925 & 352 \\
\hline & 5.25836203777553 & 808980 & 049358 \\
\hline & 9008 & 174234 & \\
\hline & 4.30252509 & 1.629 & 29815315 \\
\hline & & & 6.3966635 \\
\hline & 77729 & 73688 & \\
\hline & 6.35200586 & 039546 & \\
\hline & 23286 & 671673 & 780096 \\
\hline & 9.28693538014439 & 6.16051208266522 & 792004 \\
\hline $\mathrm{H}$ & 3582 & 355208 & 1.7 \\
\hline & 52450 & 3232325 & 619745 \\
\hline 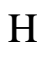 & $2.9545167 \mathrm{C}$ & 7897660 & 450396 \\
\hline & 7.69807276 & 707169 & \\
\hline $\mathrm{H}$ & & & \\
\hline $\mathrm{H}$ & 8.20427492 & 1.779 & 394457 \\
\hline $\mathrm{H}$ & 8.46110485277187 & 6815776555 & 4602445 \\
\hline $\mathrm{C}$ & 4.36074349323804 & 295249206 & 179993723762 \\
\hline $\mathrm{H}$ & 3.80315784387413 & 2.92612697876055 & \\
\hline $\mathrm{C}$ & 5.66435824340337 & -0.17679825713658 & 4.50582657699864 \\
\hline & 5.75063598772599 & -0.73906176600932 & 96877777319 \\
\hline H & 5.03622958387133 & -0.76960605332989 & 5.18779841263276 \\
\hline $\mathrm{H}$ & 6.66391741712329 & -0.09110159773599 & 4.95663087226595 \\
\hline $\mathrm{C}$ & 11.47797806038891 & 5.32464092545688 & 1.25476055861844 \\
\hline $\mathrm{H}$ & 12.35724033259006 & 5.36610318195557 & 0.60953243589145 \\
\hline & 5.74309185425885 & 6.24117506206104 & 2.43892064736856 \\
\hline $\mathrm{H}$ & 6.25875140299330 & 7.18150086155662 & 2.63870325087548 \\
\hline & 5.54406905435995 & 3.82309433090491 & 2.77540538097035 \\
\hline
\end{tabular}




\begin{tabular}{|c|c|c|c|}
\hline & 5.76043079967200 & & \\
\hline & 5.19319114778428 & -0.01834488762031 & 1.60634883571825 \\
\hline & 6.22578321342328 & 0.23988868705009 & 0.19010084461177 \\
\hline & & & \\
\hline & 10.41842801203058 & 6.22678670503094 & 336421 \\
\hline & 10.47002726534679 & 6.97235172719384 & \\
\hline & 11.40630545169824 & 4.36528531148595 & 7221477 \\
\hline & 12.23681096757499 & 3.66936622908916 & 541177 \\
\hline & 9.20257268803450 & 5.19251304874715 & 0067 \\
\hline & 6.860431514 & & 5905 \\
\hline & 7.51995978338420 & & 777848 \\
\hline & 11.07394461714896 & 1.58481567998811 & 335636 \\
\hline & 12.08 & & 8212 \\
\hline & 6.24548492420009 & 5.0387 & \\
\hline & & & \\
\hline & 4.177 & 7.18 & 976 \\
\hline & & & \\
\hline & 3.108 & & 596 \\
\hline & & & 380 \\
\hline & 3.543 & & \\
\hline & & & \\
\hline & 13.15 & & 7153 \\
\hline & 13.34 & & 384 \\
\hline & & & 7704 \\
\hline & 10.84 & 86 & 2275 \\
\hline & $10.9^{7}$ & 522 & 2843 \\
\hline & 11.45 & 672 & 0783 \\
\hline & 9.792 & & \\
\hline & 11.17 & & 5835 \\
\hline & & & \\
\hline & & & 2687 \\
\hline & 41992 & 7412 & 0012 \\
\hline & 7.613 & $6.34^{\prime}$ & 968 \\
\hline & 10.27 & 2600 & 7392 \\
\hline $\mathrm{C}$ & 11.2706 & 5360 & 5545 \\
\hline & 11.10221 & 0110565 & \\
\hline & 5.039 & 1.21 & 945187 \\
\hline $\mathrm{H}$ & 5.00199409260207 & 4542519 & 1566981 \\
\hline & & & \\
\hline & 7.81259493281472 & 8.32061161193129 & 4.29245038709680 \\
\hline & 10.50505890567193 & 0.94210044986343 & 2.65475442381048 \\
\hline $\mathrm{H}$ & 9.49176140466866 & 0.55648238935533 & 2.83109186834624 \\
\hline & & & 2.34959609235990 \\
\hline $\mathrm{H}$ & 10.46337970556705 & 1.65104111722146 & 1.81832402071045 \\
\hline & 7.82815642252449 & 3.04208960637432 & 5.11530505197146 \\
\hline
\end{tabular}




\section{$\underline{\text { Summary Tables }}$}

Table S7. Summary of relevant metric parameters for complexes 4-8.

\begin{tabular}{|c|c|c|c|c|c|}
\hline \multicolumn{7}{|c|}{ Complex } \\
\hline & $\mathbf{4}$ & $\mathbf{5}$ & $\mathbf{6}$ & $\mathbf{7}$ & $\mathbf{8}$ \\
\hline Fe1-C1 & $3.834(3)$ & $1.792(1)$ & $3.983(3)$ & 3.811 & $1.911(4)$ \\
\hline Fe1-H1 & --- & $1.76(3)$ & --- & $1.52(5)$ & --- \\
\hline Fe1-P1 & $2.254(5)$ & $2.1307(7)$ & $2.1547(1)$ & $2.145(1)$ & $2.113(1)$ \\
\hline Fe1-P2 & $2.330(6)$ & $2.2627(8)$ & $2.1584(2)$ & $2.161(1)$ & $2.221(1)$ \\
\hline Fe1-P3 & $2.318(6)$ & $2.2522(6)$ & $2.1688(2)$ & $2.186(1)$ & $2.217(1)$ \\
\hline Fe1-Fe2 & $7.666(6)$ & $2.6776(6)$ & $7.966(5)$ & $4.758(1)$ & $5.8861(9)$ \\
\hline Fe1-Br1 & $2.351(4)$ & --- & --- & --- & --- \\
\hline Fe1-N1 & --- & --- & --- & $1.794(3)$ & $1.821(3)$ \\
\hline N1-N2 & --- & --- & $1.107(5)$ & $1.167(4)$ & $1.113(5)$ \\
\hline Fe2-P4 & --- & --- & --- & $2.142(1)$ & $2.179(1)$ \\
\hline Fe2-P5 & --- & --- & --- & $2.160(1)$ & $2.157(1)$ \\
\hline Fe2-P6 & --- & --- & --- & $2.182(1)$ & $2.178(1)$ \\
\hline Fe2-H2 & --- & --- & --- & $1.47(5)$ & $1.54(3)$ \\
\hline Fe2-H3 & --- & --- & --- & --- & $1.53(3)$ \\
\hline Fe2-N2 & --- & --- & --- & $1.804(3)$ & --- \\
\hline Fe2-N3 & --- & --- & --- & --- & $1.800(3)$ \\
\hline N3-N4 & --- & --- & --- & --- & $1.115(5)$ \\
\hline Fe1-C1-Fe2 & --- & $96.66(1)$ & --- & --- & --- \\
\hline H1-Fe1-C1 & --- & $82.3(8)$ & --- & --- & --- \\
\hline P2-Fe1-P3 & $121.7(2)$ & $116.14(3)$ & $146.72(5)$ & $106.28(5)$ & $123.68(5)$ \\
\hline P5-Fe2-P6 & --- & --- & --- & $107.95(5)$ & $151.36(5)$ \\
\hline P1-C3-C4 & $123.1(1)$ & $141.42(1)$ & $120.0(2)$ & --- & $128.6(3)$ \\
\hline C1-C2-C3 & $118.7(1)$ & $117.52(9)$ & $119.86(2)$ & $120.1(3)$ & $116.1(3)$ \\
\hline P1-Fe1-N1 & --- & --- & $105.22(1)$ & $99.6(1)$ & $176.2(1)$ \\
\hline C1-C2-C3-P1 & $5.9(1)$ & $0.36(8)$ & $0.8(2)$ & $29.8(5)$ & $5.3(4)$ \\
\hline & & & & & \\
\hline
\end{tabular}


Table S7. Summary of statistics for diffraction data relevant for complexes 4-5.

\begin{tabular}{|c|c|c|}
\hline & 4 & 5 \\
\hline $\mathrm{CCDC}$ & 1997107 & 1997108 \\
\hline Empirical formula & $\mathrm{C}_{55} \mathrm{H}_{56} \mathrm{P}_{6} \mathrm{Fe}_{2} \mathrm{Br}_{2}$ & $\mathrm{C}_{65} \mathrm{H}_{100} \mathrm{Fe}_{2} \mathrm{P}_{6}$ \\
\hline Formula weight & 1174.33 & 1178.96 \\
\hline Temperature/K & 99.99 & 100 \\
\hline Crystal system & monoclinic & monoclinic \\
\hline Space group & $\mathrm{C} 2 / \mathrm{c}$ & $\mathrm{C} 2 / \mathrm{c}$ \\
\hline $\mathrm{a} / \AA$ & $31.847(10)$ & $14.878(4)$ \\
\hline $\mathrm{b} / \AA ̊ \AA$ & $11.095(2)$ & $16.350(5)$ \\
\hline$c / \AA$ & $16.608(4)$ & $25.612(5)$ \\
\hline$\alpha^{/}$ & 90 & 90 \\
\hline$\beta /{ }^{\circ}$ & $93.410(15)$ & $90.577(15)$ \\
\hline$\gamma /{ }^{\circ}$ & 90 & 90 \\
\hline Volume $/ \AA^{3}$ & $5858(3)$ & $6230(3)$ \\
\hline $\mathrm{Z}$ & 4 & 4 \\
\hline$\rho_{\text {calc }} \mathrm{g} / \mathrm{cm}^{3}$ & 1.332 & 1.257 \\
\hline$\mu / \mathrm{mm}^{-1}$ & 7.365 & 5.469 \\
\hline $\mathrm{F}(000)$ & 2392 & 2528 \\
\hline Crystal size/ $/ \mathrm{mm}^{3}$ & $0.18 \times 0.1 \times 0.01$ & $0.301 \times 0.25 \times 0.24$ \\
\hline Radiation & $\mathrm{CuK} \alpha(\lambda=1.54178)$ & $\mathrm{CuK} \alpha(\lambda=1.54178)$ \\
\hline $2 \Theta$ range for data collection ${ }^{\circ}$ & 5.56 to 152.342 & 6.902 to 160.822 \\
\hline Index ranges & $-37 \leq \mathrm{h} \leq 39,-13 \leq \mathrm{k} \leq 13,-20 \leq 1 \leq 18$ & $-18 \leq \mathrm{h} \leq 18,-20 \leq \mathrm{k} \leq 20,-29 \leq 1 \leq 32$ \\
\hline Reflections collected & 41187 & 58255 \\
\hline Independent reflections & $5568[$ Rint $=0.1228$, Rsigma $=0.0826]$ & $6723[$ Rint $=0.0496$, Rsigma $=0.0260]$ \\
\hline Data/restraints/parameters & $5568 / 0 / 303$ & $6723 / 0 / 343$ \\
\hline Goodness-of-fit on $\mathrm{F}^{2}$ & 1.076 & 1.024 \\
\hline Final $\mathrm{R}$ indexes $[\mathrm{I}>=2 \sigma(\mathrm{I})]$ & $\mathrm{R} 1=0.1876, \mathrm{wR} 2=0.3857$ & $\mathrm{R} 1=0.0296, \mathrm{wR} 2=0.0738$ \\
\hline Final $\mathrm{R}$ indexes [all data] & $\mathrm{R} 1=0.2340, \mathrm{wR} 2=0.4128$ & $\mathrm{R} 1=0.0315, \mathrm{wR} 2=0.0749$ \\
\hline Largest diff. peak/hole / e $\AA^{-3}$ & $2.17 /-1.20$ & $0.36 /-0.27$ \\
\hline
\end{tabular}


Table S8. Summary of statistics for diffraction data relevant for complexes 6-7.

\begin{tabular}{|c|c|c|}
\hline & 6 & 7 \\
\hline $\mathrm{CCDC}$ & 1997109 & 1997110 \\
\hline Empirical formula & $\mathrm{C}_{55} \mathrm{H}_{80} \mathrm{Fe}_{2} \mathrm{~N}_{4} \mathrm{P}_{6}$ & $\mathrm{C}_{60} \mathrm{H}_{92} \mathrm{Fe}_{2} \mathrm{~N}_{2} \mathrm{P}_{6}$ \\
\hline Formula weight & 1094.75 & 1138.87 \\
\hline Temperature/K & 100 & 100 \\
\hline Crystal system & monoclinic & orthorhombic \\
\hline Space group & $\mathrm{C} 2 / \mathrm{c}$ & Pbca \\
\hline $\mathrm{a} / \AA$ & $32.05(2)$ & $21.364(7)$ \\
\hline $\mathrm{b} / \AA$ & $11.137(11)$ & $18.351(4)$ \\
\hline $\mathrm{c} / \AA$ & $16.323(12)$ & $29.604(5)$ \\
\hline$\alpha /^{\circ}$ & 90 & 90 \\
\hline$\beta /{ }^{\circ}$ & $94.19(3)$ & 90 \\
\hline$\gamma /{ }^{\circ}$ & 90 & 90 \\
\hline Volume $/ \AA^{3}$ & $5811(8)$ & $11606(5)$ \\
\hline Z & 4 & 8 \\
\hline$\rho_{\text {calc }} \mathrm{g} / \mathrm{cm}^{3}$ & 1.251 & 1.304 \\
\hline$\mu / \mathrm{mm}^{-1}$ & 0.702 & 5.864 \\
\hline $\mathrm{F}(000)$ & 2320 & 4864 \\
\hline Crystal size $/ \mathrm{mm}^{3}$ & $0.344 \times 0.168 \times 0.146$ & $0.371 \times 0.268 \times 0.161$ \\
\hline Radiation & $\operatorname{MoK} \alpha(\lambda=0.71073)$ & $\mathrm{CuK} \alpha(\lambda=1.54178)$ \\
\hline $2 \Theta$ range for data collection ${ }^{\circ}$ & 3.872 to 66.634 & 5.97 to 161.414 \\
\hline Index ranges & $-45 \leq \mathrm{h} \leq 47,-16 \leq \mathrm{k} \leq 15,-23 \leq 1 \leq 24$ & $-27 \leq \mathrm{h} \leq 25,-22 \leq \mathrm{k} \leq 22,-35 \leq 1 \leq 37$ \\
\hline Reflections collected & 66591 & 174786 \\
\hline Independent reflections & $9410[$ Rint $=0.0358$, Rsigma $=0.0315]$ & $12333[$ Rint $=0.0980$, Rsigma $=0.0422]$ \\
\hline Data/restraints/parameters & $9410 / 38 / 330$ & $12333 / 0 / 683$ \\
\hline Goodness-of-fit on $\mathrm{F}^{2}$ & 1.077 & 1.133 \\
\hline Final $R$ indexes $[I>=2 \sigma(I)]$ & $\mathrm{R} 1=0.0801, \mathrm{wR} 2=0.1846$ & $\mathrm{R} 1=0.0699, \mathrm{wR} 2=0.1287$ \\
\hline Final $\mathrm{R}$ indexes [all data] & $\mathrm{R} 1=0.1020, \mathrm{wR} 2=0.1970$ & $\mathrm{R} 1=0.0879, \mathrm{wR} 2=0.1374$ \\
\hline Largest diff. peak/hole / e $\AA^{-3}$ & $2.95 /-1.60$ & $0.64 /-0.58$ \\
\hline
\end{tabular}


Table S9. Summary of statistics for diffraction data relevant for complex $\mathbf{8}$.

\begin{tabular}{|c|c|}
\hline & 8 \\
\hline $\mathrm{CCDC}$ & 1997111 \\
\hline Empirical formula & $\mathrm{C}_{55} \mathrm{H}_{78} \mathrm{Fe}_{2} \mathrm{~N}_{4} \mathrm{P}_{6}$ \\
\hline Formula weight & 1092.73 \\
\hline Temperature/K & 100 \\
\hline Crystal system & monoclinic \\
\hline Space group & $\mathrm{P} 21 / \mathrm{n}$ \\
\hline $\mathrm{a} / \AA$ & $18.123(3)$ \\
\hline $\mathrm{b} / \AA$ & $17.599(3)$ \\
\hline $\mathrm{c} / \AA$ & $18.209(2)$ \\
\hline$\alpha /^{\circ}$ & 90 \\
\hline$\beta /{ }^{\circ}$ & $108.130(10)$ \\
\hline$\gamma /{ }^{\circ}$ & 90 \\
\hline Volume $/ \AA^{3}$ & $5519.0(15)$ \\
\hline Z & 4 \\
\hline$\rho_{\text {calc }} \mathrm{g} / \mathrm{cm}^{3}$ & 1.315 \\
\hline$\mu / \mathrm{mm}^{-1}$ & 6.158 \\
\hline $\mathrm{F}(000)$ & 2312 \\
\hline Crystal size $/ \mathrm{mm}^{3}$ & $0.257 \times 0.086 \times 0.079$ \\
\hline Radiation & $\mathrm{CuK} \alpha(\lambda=1.54178)$ \\
\hline $2 \Theta$ range for data collection $/{ }^{\circ}$ & 6.008 to 161.41 \\
\hline Index ranges & $-18 \leq \mathrm{h} \leq 22,-22 \leq \mathrm{k} \leq 19,-23 \leq 1 \leq 22$ \\
\hline Reflections collected & 50951 \\
\hline Independent reflections & $11649[$ Rint $=0.0910$, Rsigma $=0.0702]$ \\
\hline Data/restraints/parameters & $11649 / 1 / 632$ \\
\hline Goodness-of-fit on $\mathrm{F}^{2}$ & 1.061 \\
\hline Final $R$ indexes $[\mathrm{I}>=2 \sigma(\mathrm{I})]$ & $\mathrm{R} 1=0.0633, \mathrm{wR} 2=0.1696$ \\
\hline Final R indexes [all data] & $\mathrm{R} 1=0.0836, \mathrm{wR} 2=0.1868$ \\
\hline Largest diff. peak/hole / e $\AA^{-3}$ & $1.17 /-0.64$ \\
\hline
\end{tabular}




\section{References:}

1. Mankad, N. P.; Rivard, E.; Harkins, S. B.; Peters, J. C., Structural Snapshots of a Flexible $\mathrm{Cu}_{2} \mathrm{P}_{2}$ Core that Accommodates the Oxidation States $\mathrm{Cu}^{\mathrm{I}} \mathrm{Cu}^{\mathrm{I}}, \mathrm{Cu}^{1.5} \mathrm{Cu}^{1.5}$, and $\mathrm{Cu}^{\mathrm{II}} \mathrm{Cu}^{\mathrm{II}}$. J. Am. Chem. Soc. 2005, 127 (46), 16032-16033.

2. Chilton, N. F.; Anderson, R. P.; Turner, L. D.; Soncini, A.; Murray, K. S., PHI: A powerful new program for the analysis of anisotropic monomeric and exchange-coupled polynuclear d- and f-block complexes. J. Comp. Chem. 2013, 34 (13), 1164-1175.

3. APEX-II Version 2 User Manual, M86-E01078. Bruker Analytical X-ray Systems: Madison, WI, 2006.

4. Sheldrick, G., SADABS (version 2008/1): Program for Absorption Correction for Data from Area Detector Frames. University of Göttingen: 2008.

5. Sheldrick, G., A short history of SHELX. Acta Crystallographica Section A 2008, 64 (1), 112122.

6. Sheldrick, G., Crystal structure refinement with SHELXL. Acta Crystallographica Section C 2015, 71 (1), 3-8.

7. Dolomanov, O. V.; Bourhis, L. J.; Gildea, R. J.; Howard, J. A. K.; Puschmann, H., OLEX2: a complete structure solution, refinement and analysis program. J. Appl. Crystallogr. 2009, 42 (2), 339-341. 8. Neese, F., The ORCA program system. WIREs Computational Molecular Science 2012, 2 (1), 73 78.

9. Pettersen, E. F.; Goddard, T. D.; Huang, C. C.; Couch, G. S.; Greenblatt, D. M.; Meng, E. C.; Ferrin, T. E., UCSF Chimera-A visualization system for exploratory research and analysis. J. Comput. Chem. 2004, 25 (13), 1605-1612.

10. Neese, F., Prediction and interpretation of the ${ }^{57} \mathrm{Fe}$ isomer shift in Mössbauer spectra by density functional theory. Inorg. Chim. Acta 2002, 337, 181-192.

11. Thompson, N. B.; Green, M. T.; Peters, J. C., Nitrogen Fixation via a Terminal Fe(IV) Nitride. J. Am. Chem. Soc. 2017, 139 (43), 15312-15315. 